\title{
Development Of Sample
} Handling And Analytical Expertise For The Stardust Comet Sample Return

J.P. Bradley, S. Bajt, S. Brennan, G.A. Graham, P.G. Grant, I.D. Hutcheon, H. Ishii, P. Pianetta, A. Toppani, A.J. Westphal.

February $10^{\text {th }} 2006$

LDRD Project (03-ERI-007) Final Report 


\section{Disclaimer}

This document was prepared as an account of work sponsored by an agency of the United States Government. Neither the United States Government nor the University of California nor any of their employees, makes any warranty, express or implied, or assumes any legal liability or responsibility for the accuracy, completeness, or usefulness of any information, apparatus, product, or process disclosed, or represents that its use would not infringe privately owned rights. Reference herein to any specific commercial product, process, or service by trade name, trademark, manufacturer, or otherwise, does not necessarily constitute or imply its endorsement, recommendation, or favoring by the United States Government or the University of California. The views and opinions of authors expressed herein do not necessarily state or reflect those of the United States Government or the University of California, and shall not be used for advertising or product endorsement purposes.

Auspices Statement

This work was performed under the auspices of the U. S. Department of Energy (DOE) by the University of California, Lawrence Livermore National Laboratory (LLNL) under Contract No. W-7405-Eng-48. The project (03-ERI-007) was funded by the Laboratory Directed Research and Development Program at LLNL. 
"...a certain fraction of the cost of mounting sample return missions should be set aside to stimulate instrument development here on Earth. This would maximize the integrated scientific return from the missions; perhaps more important such investment would increase the benefits to technology and science as a whole. Unless such a policy is adopted, I am afraid that our community will not play as key a role in the future development of analytical techniques as it could."

R.M. Walker (1991) 


\title{
FY05 LDRD Final Report Development of Sample Handling and Analytical Expertise for the Stardust Cometary Return LDRD Project Tracking Code: 03-ERI-007 John P. Bradley, Principal Investigator
}

\begin{abstract}
NASA's Stardust mission returned to Earth in January 2006 with "fresh" cometary particles from a young Jupiter family comet. The cometary particles were sampled during the spacecraft flyby of comet 81P/Wild-2 in January 2004, when they impacted low-density silica aerogel tiles and aluminum foils on the sample tray assembly at approximately $6.1 \mathrm{~km} / \mathrm{s}$. This LDRD project has developed extraction and sample recovery methodologies to maximize the scientific information that can be obtained from the analysis of natural and man-made nano-materials of relevance to the LLNL programs.
\end{abstract}

\section{Introduction/Background}

The Stardust mission launched in February 1999 and is the first US "solid matter" sample return mission since Apollo 17 in 1973. The primary purpose of STARDUST was to collect samples of comet Wild-2 and return them to Earth. A secondary purpose was to collect samples of contemporary interstellar dust, detected by the Ulysses and Galileo spacecraft, moving through the solar system in a dust stream parallel to the flow of the interstellar gas. The Aerogel collectors on STARDUST were deployed for interstellar dust collection and the spacecraft successfully passed through the tail of Comet Wild-2 resulting in the capture of numerous micro- and nano-meter sized cometary particles in January 2004 (Fig.1) (Brownlee et al., 2004). The Sample Return Capsule successfully re-entered Earth atmosphere on January $15^{\text {th }}, 2006$.
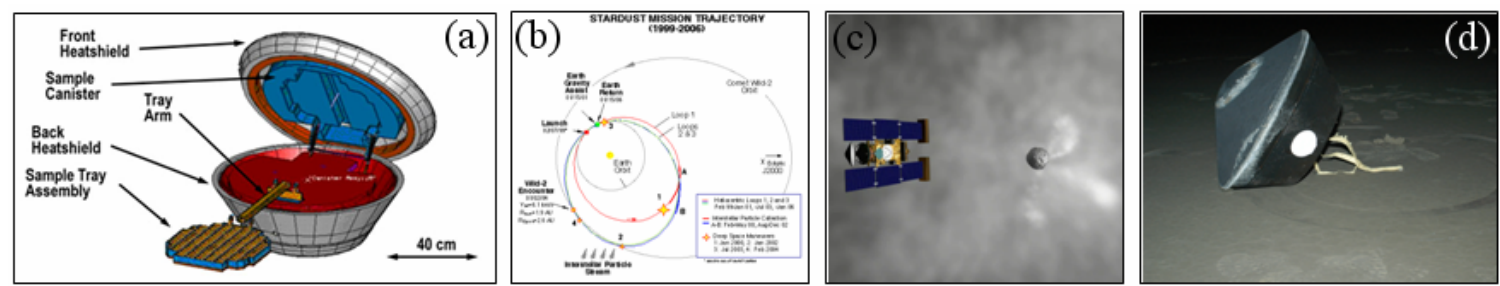

Figure 1. (a) Schematic diagram of the Stardust sample return capsule. (b) The Stardust mission trajectory. (c) An artist's impression of the spacecraft during the encounter with comet Wild-2. (d) The sample return capsule after it had landed at the Utah Test and Training Range. (All images courtesy of NASA)

The Stardust samples are unique in three respects. First, the entire (microgrammass) sample will consist entirely of individual particles ranging from nanometers to micrometers in size. The mission aim was to collect approximately 100 interstellar grains and at least 1000 comet grains. Second, the grains will be embedded in aerogel. The interstellar dust grains will have entered the aerogel at approximately $12 \mathrm{~km} / \mathrm{sec}$ and the comet grains at $6.1 \mathrm{~km} / \mathrm{sec}$. Each grain will be located at the narrow end of a carrot-shaped track $1-2 \mathrm{~mm}$ in length within the aerogel (Fig. 2). 
Third, astronomical data and data from cosmic dust collected in the stratosphere indicate that the Stardust grains will likely be composed of nano-materials requiring development of state-of-the-art sample handling and analytical facilities (e.g. Bradley et al., 2004).

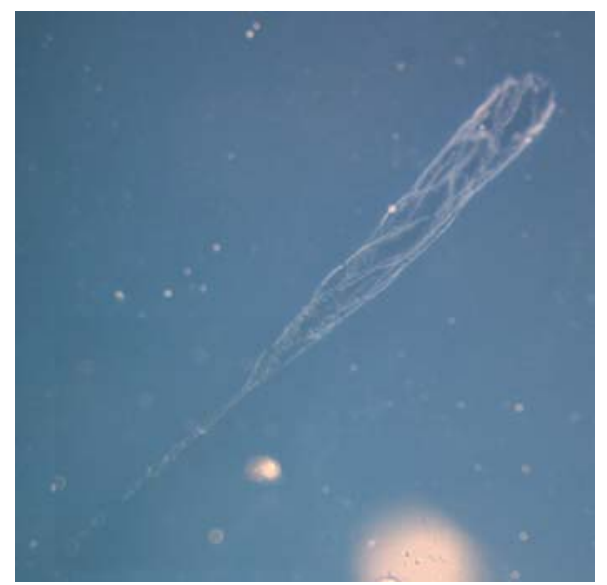

Figure 2. Reflected light optical image of an impact track preserved in silica aerogel.

The Stardust samples will offer new insights into early solar system process. Comets are unique small bodies that spend most of their lifetimes at extreme heliocentric distances. These icy bodies are believed to contain preserved interstellar dust as well as the first solids condensed in the inner solar system. Thus comet samples can be considered as "time capsules" from the earliest stages of solar system formation. Interstellar dust is one of the fundamental building blocks from which the solar system formed. (Most of the condensed elements in the solar system were carried within interstellar dust grains immediately prior to the collapse of the solar nebula). Much of what we know about interstellar dust has been inferred from astronomical observations and from laboratory studies of exotic presolar grains in meteorites (e.g. SiC, nano-diamonds). But these grains represent a tiny subset that are refractory enough to have survived alteration on the asteroidal parent bodies of meteorites and the acid digestion process used to extract them from the meteorites. Analysis of even a few contemporary interstellar dust grains from Stardust will greatly improve our understanding of the solid particulate composition of the interstellar medium and provide fundamental insight about astronomical phenomena such as light scattering, extinction, polarization, and the relative abundances of the elements in interstellar molecular cloud environments.

Stardust is the first of the next generation of space missions that will return material to Earth for detail analysis. Future missions are likely to use silica aerogel as the primary capture medium due to the ability to collect material at hypervelocity speeds while reducing the effects of shock and thermal alteration. However prior to analysis it is critical that the captured material is suitably prepared. Therefore the principal aim of this LDRD project was to develop sample handling and extraction techniques to recovery material from silica aerogel. This LDRD project also aimed to assess analytical techniques suitable for detailed characterization of the natural nanomaterials including emerging technologies, such as focused ion beam microscopy. The proposal aligned with the fundamental Science and Applied Technology mission goal of Lawrence Livermore National Laboratory with specific reference to Astrophysics \& Space Science as the analysis to be performed on Stardust samples will support the exploration of small solar system bodies, including Kuiper Belt Objects like comet Wild-2. Any technological development for the 
recovery to material from aerogel has the potential application to NIF experiments seeking to recover particulate hypervelocity ejecta from aerogel generated during laser shots. Any development of integrated analysis methodology use to investigate Stardust samples could be applied to interrogate interdicted materials for forensic studies in support of national security initiatives.

\section{Research Activities}

To develop sample handling techniques and analytical expertise for Stardust it has been necessary to study analogue materials. NASA's Orbital Debris Collector Experiment (ODCE) exposed silica aerogel tiles as part of the Mir Environment Effects Package that was exposed on the Mir space station for 18 months (Hörz et al., 2000). For this project a number of ODCE tiles were supplied for the developmental work. In addition to the space exposed aerogel tiles, laboratory simulated impacts into aerogel using light-gas-gun accelerated minerals and meteoritic powders were also used. Interplanetary dust particles collected in the stratosphere have provided relatively pristine material to investigate using the extensive analytical facilities at Lawrence Livermore National Laboratory. The key research activities for the project are summarized as follows:

Impact Track Extraction and Sample Recovery

A number of techniques have been evaluated for the recovery of impact tracks, including the use of the $355 \mathrm{~nm}$ micro-focused laser cutting workstation. However the most significant advance was the use of diamond micro-blades under ultrasonic oscillation to enable high precision cutting of aerogel (Figure 3 ). High precision focused ion beam (FIB) milling has been used to extract particles from aerogel (Figure 4).

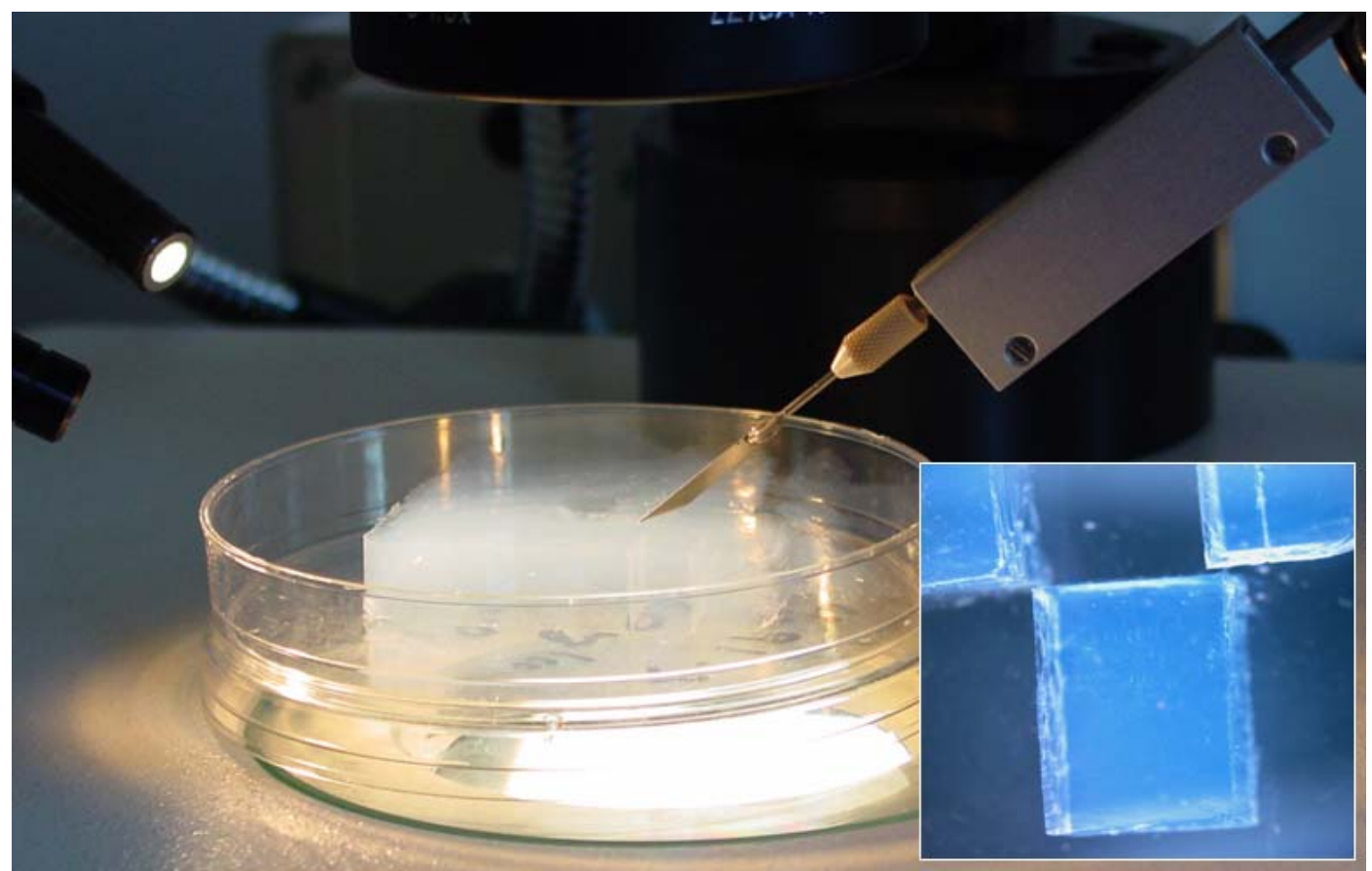

Figure 3. The ultra-sonic micro-blade cutting device developed under this LDRD project. The insert shows a resulting aerogel cube extracted after micro-blade cutting. 

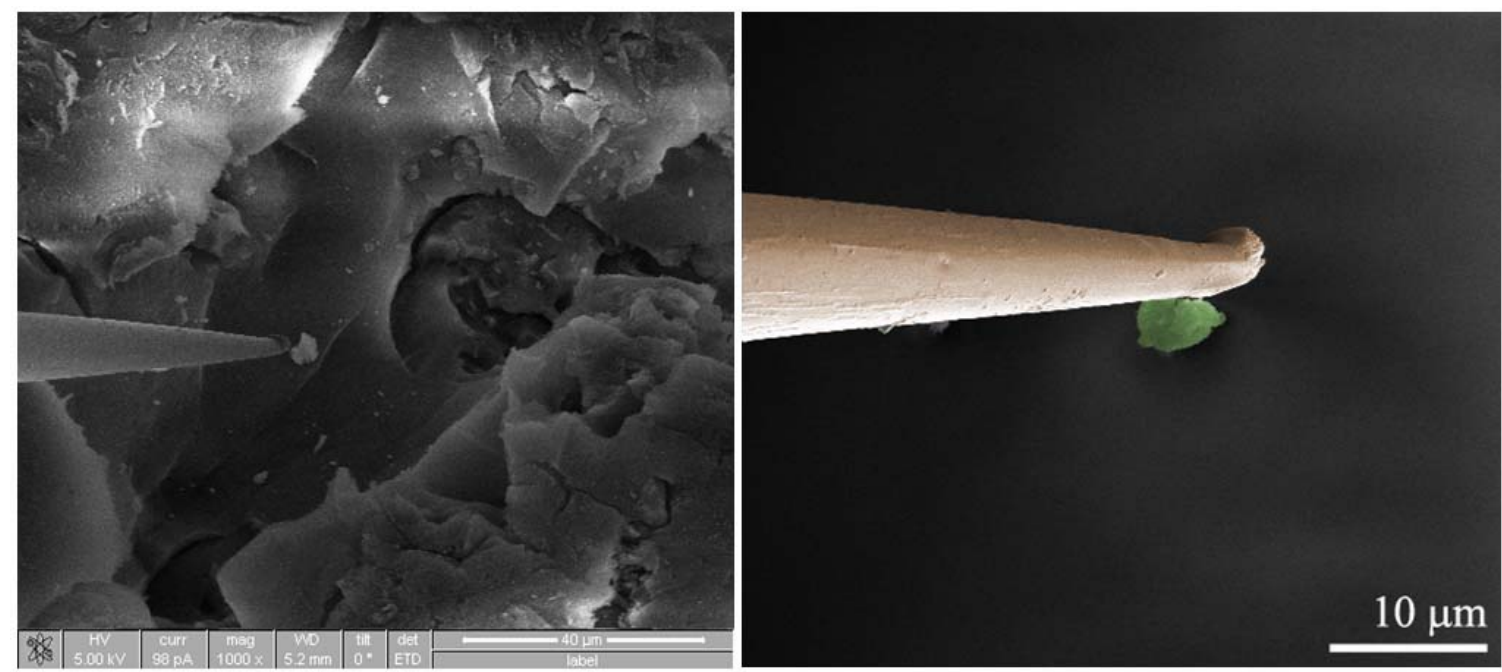

Figure 4. Shows the extraction of a particle from aerogel using the in-situ micromanipulator fitted inside the FIB chamber.

Analytical Expertise

Preliminary characterization of material encapsulated within the Stardust aerogel collectors is a key requirement. A fundamental analytical difficulty is that the particulate debris is surrounded by aerogel, making imaging and analysis using certain techniques challenging if not impossible. Both the nuclear microprobe at Lawrence Livermore National Laboratory and the hard $\mathrm{x}$-ray microprobe at Stanford Synchrotron Radiation Laboratory have proved to be well suited to in-situ mapping. After preliminary in-situ measurements, the ability to further interpret the nature of the materials will depend on the ability to recover particulate debris. Developmental FIB work has enabled detailed analysis of Stardust analogues using TEM, synchrotron infrared micro-spectroscopy and NanoSIMS techniques.

\section{Results/Technical Outcome}

The research carried out under the project has resulted in a number of publications. A selected number of these papers are submitted with this final report document to highlight the technical achievements.

In addition to the results highlight in the published papers, this LDRD project has resulted in the formation of a multi-institutional collaboration. Astromaterials is a relatively new branch of astrophysics which draws on a number of scientific fields, e.g. geology, cosmochemistry, theoretical astrophysics. To meet the challenges of this new field, a consortium of national laboratories and university research groups in the Bay Area was formed in 2002 to exploit the wide range of state-of-the-art analytical instrumentation and diverse knowledge base. Founding members of BayPAC (Bay Area Particle Analysis Consortium) are: IGPP-LLNL with extensive experience in microbeam techniques and the lead institution; Lawrence Berkeley Laboratory with synchrotron infrared spectroscopy capabilities; Stanford Synchrotron Radiation Laboratory at the Stanford Linear Accelerator Center with X-ray microprobe capabilities and the Space Science Laboratory at UC Berkeley with experience in theoretical astrophysics (Figure 5). Since its inception, BayPAC members have published a number of high profile papers combining traditional astrophysics with astromaterials observations (e.g. Westphal \& Bradley 2004 and Bradley \& Dai, 2004). The BayPAC model was highlighted in a 2005 National Research Council of the National Academies review of midsize facilities. 


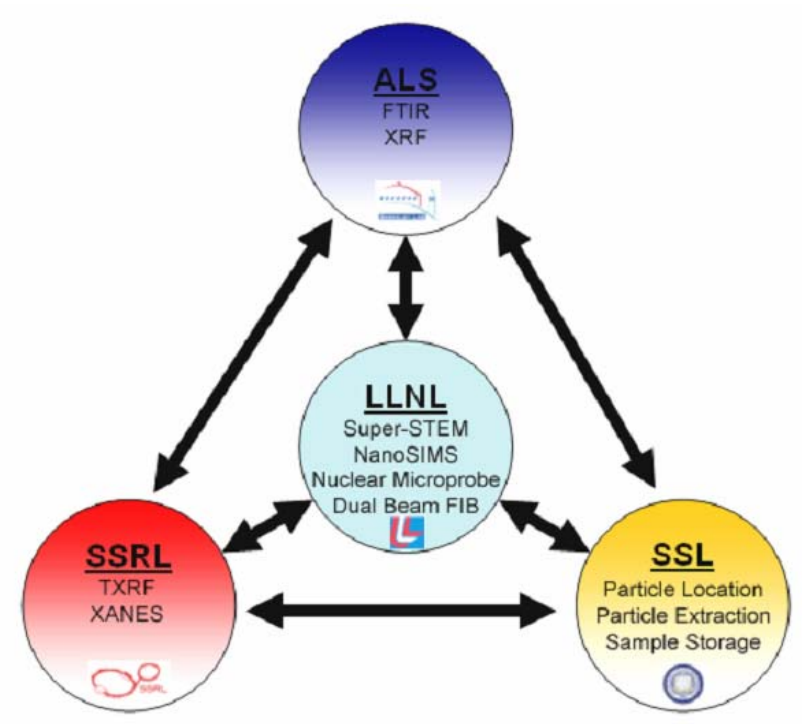

Figure 5. A "Hub \& Spoke" diagram showing the interaction of BayPAC institutions.

A key aim of the project was to develop techniques for the extraction of impact tracks generated in aerogel. Ishii et al. (2005a) discussed the use of ultra-sonic diamond micro-blades to recovery impact tracks from silica aerogel. The ultra-sonic micro-blade technology was developed at Lawrence Livermore National Laboratory under this project and has been submitted for a patent (ROI \#IL-11590).

In addition to track recovery the project has extensive evaluated a range of analytical techniques for both in-situ characterization and recovery of embedded material. Graham et al. (2004) discussed the application of the detection techniques associated with the nuclear microprobe to characterize material embedded in aerogel and the using of FIB microscopy to subsequently recovery embedded particles. Ishii et al. (2005b) discussed the development of single particle analysis for trace element composition using the synchrotron $x$-ray micro-spectroscopy beamline at Stanford Synchrotron Radiation Laboratory.

Missions such as Stardust will only return a limited amount of material therefore it is important that the maximum information in terms of mineralogical, chemical and isotopic composition be obtained. As a result over the past 3 years, we have been developing an integrated approach to the microanalysis. A key analytical technology for such studies is FIB microscopy (Graham et al., 2005). FIB enables the recovery of regions of interest within a single dust grain after initial isotope imaging using the NanoSIMS. The FIB recovered section can then be examined using the TEM and infrared microscope before re-examination in the NanoSIMS (Figure 6). Floss et al. (2004) and Bradley et al. (2005) give examples of the integrated approach to astromaterials research. 


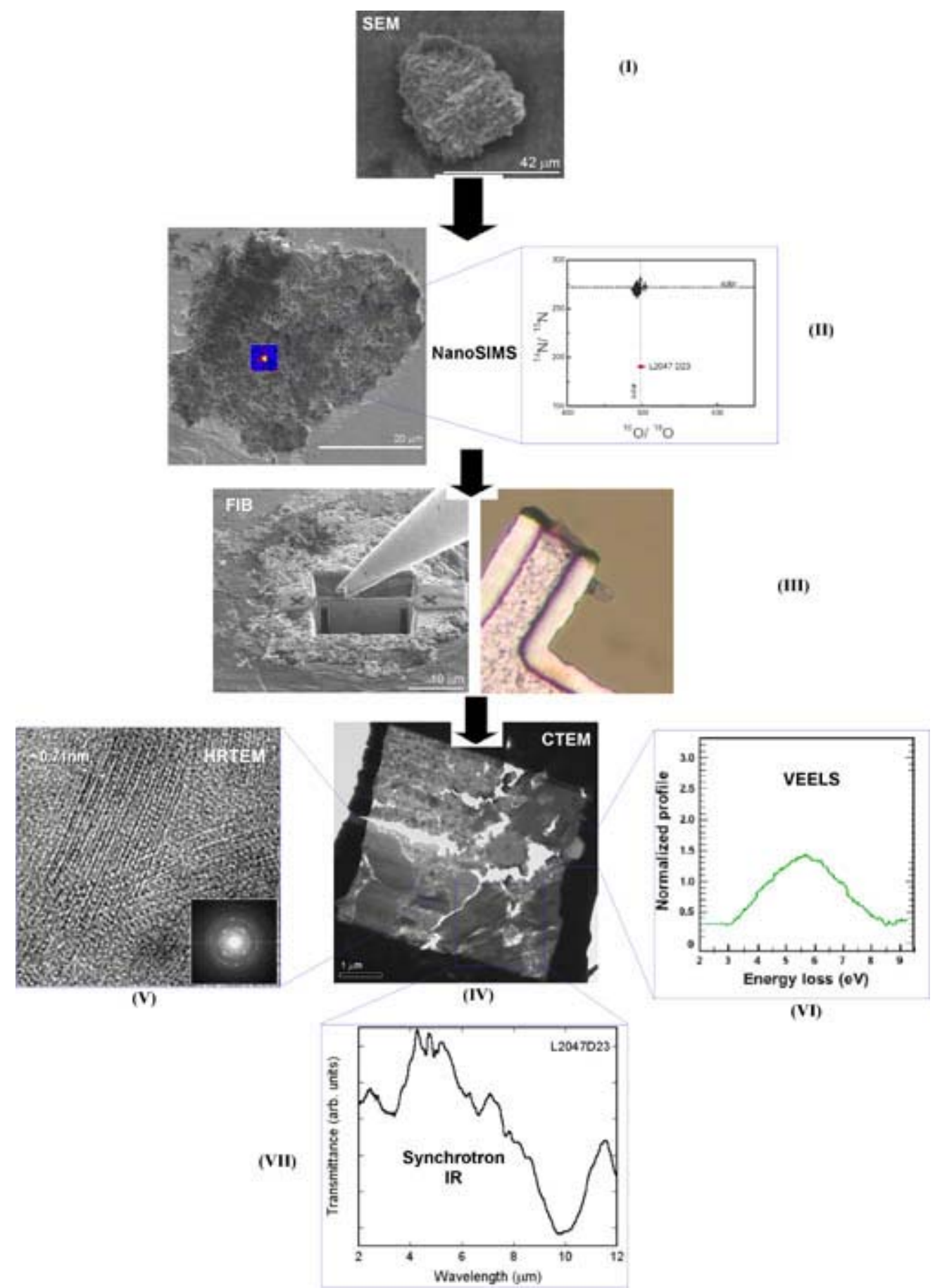

Figure 6. Integrated microanalysis for a hydrous IDP. (I) Initial image and bulk characterization using SEM (image courtesy of NASA) (II) NanoSIMS imaging and isotopic analysis. (III) FIB recovery of the material containing the isotopic anomaly. (IV-VI) Detailed TEM analysis. (VII) Synchrotron IR studies.

\section{Summary}

LDRD project 03-ERI-007 has resulted in the development of sample handling/preparation and analytical expertise for astromaterial sample return missions resulting in the recovery of micro-grams and nano-grams of material. The major accomplishments of the project are summarized as follows: (i) Development of 
an extraction system based on ultrasonic oscillation of diamond micro-blades for the recovery of particles embedded in low-density materials (Ishii et al. 2005). The method of material recovery has resulted in a patent submission ROI \#IL-11590. (ii) Detailed assessment of a wide range of analytical techniques for the analysis of astromaterials. During this assessment a new design for an enhanced X-ray detector for elemental analysis has resulted in a patent submission ROI \#IL-11435. (iii) The formation of the BayPAC multi-institutional collaboration between Lawrence Livermore National Laboratory, Stanford Synchrotron Radiation Laboratory, Lawrence Berkeley National Laboratory and University of California at Berkeley. BayPAC was formed to maximize the diverse range of analytical and theoretical expertise in astromaterial and astrophysics research in the San Francisco Bay Area.

\section{Publications}

The following publications resulted from the research performed under LDRD 03-ERI007.

Bajt S., Graham G.A., Bradley J., Westphal A.J., Butterworth A.L. and Martin M.C. (2004) Mid- and Far-infrared spectroscopy at the Advanced Light Source. . In Lunar and Planetary Science XXXV, Abstract \#2127, Lunar and Planetary Institute, Houston (CD-ROM). UCRL-ABS-201973.

Bradley J., Dai Z.R., Erni R., Browning N., Graham G., Weber P., Smith J., Hutcheon I., Ishii H., Bajt S., Floss C., Stadermann F. and Sandford S. (2005) The astronomical 2175 angstrom feature in interplanetary dust particles. Science 307, 244-247. UCRL-JRNL-205703.

Floss C., Stadermann F. J., Bradley J., Dai Z.R., Bajt S. and Graham G. A. (2004) Carbon and nitrogen isotopic anomalies in an anhydrous interplanetary dust particle. Science 303, 1355-1358. UCRL-JRNL-201533.

Graham G.A., Grant P.G., Chater R.J., Westphal A.J., Kearsley A.T., Snead C., Dominguez G., Butterworth A.L., McPhail D.S., Bench G. and Bradley J.P. (2004) Investigation of ion beam techniques for the in situ analysis and exposure of particles encapsulated by silica aerogel: applicability for Stardust. Meteoritics and Planetary Science. 39, 1461-1474. UCRL-JC-155390.

Graham G.A., Bradley J.P., Bernas M., Stroud R.M., Dai Z.R., Floss C., Stadermann F.J., Snead C.J. and Westphal A.J. (2004) Focused ion beam recovery and analysis of interplanetary dust particles (IDPs) and Stardust Analogues. In Lunar and Planetary Science $X X X V$, Abstract \#2127, Lunar and Planetary Institute, Houston (CD-ROM). UCRL-ABS-201974.

Graham G.A., Sheffield-Parker J., Bradley J.P., Kearsley A.T., Dai Z.R., Mayo S.C., Teslich N., Snead C., Westphal A.J. and Ishii H. (2005) Electron beam analysis of micrometeoroids captured in aerogel as Stardust analogues. In Lunar and Planetary Science XXXVI, Abstract \#2078, Lunar and Planetary Institute, Houston (CD-ROM).

Graham G.A., Bernas M., Dai Z.R., Bradley J.P., Smith J.B., Weber P.K., Ishii H., Hutcheon I.D. (2005) Recovery of isotopic hotspot in interplanetary dust particles by focused ion beam microscopy: Bridging the gap between NanoSIMS and (S)TEM. Microsc. Microanal. 11(suppl. 2), 1390-1392. UCRL-ABS-21065 
Ishii H.A., Graham G.A., Kearsley A.T., Grant P.G., Snead C.J. and Bradley J.P. (2005) Rapid extraction of dust impact tracks from silica aerogel by ultrasonic microblades. Meteorit. Planet. Sci., 40, 1741-1747. UCRL-JRNL-209950.

Ishii H.A., Brennan S., Luening K., Pianetta P., Bradley J.P., Snead C.J. and Westphal A.J. (2005) Hard x-ray spectro-microscopy techniques at SSRL for astromaterials analysis. 36th Lunar and Planetary Science Conference (League City, TX, March 2005), CD-ROM: Abstract 1393. UCRL-ABS-208887.

Ishii H.A., Graham G.A., Kearsley A.T., Grant P.G., Snead C.J., and Bradley J.P. (2005) Ultrasonic micro-blades for the rapid extraction of impact tracks from aerogel, 36th Lunar and Planetary Science Conference (League City, TX, March 2005), CD-ROM: Abstract 1387. UCRL-ABS-208888.

Westphal A.J., Snead C., Butterworth A., Graham G.A., Bradley J.P., Bajt S., Grant P.G., Bench G., Brennan S. and Piannetta P. (2004) Aerogel keystones: Extraction of complete hypervelocity impact events from aerogel collectors. Meteoritics and Planetary Science 39, 1375-1386. UCRL-JP-200884.

\section{Acknowledgements}

Dr. Friedrich Hörz (NASA Johnson Space Center) is thanked for supplying the lowEarth orbit exposed aerogel samples used in this study. Mr. Anton Kearsley (Natural History Museum, London) is thanked for supplying mineral standards and meteoritic materials used in this project.

\section{References Relating to the Final Report}

Bradley, J.P. and Dai, Z.R. (2004) Mechanism of formation form glass with embedded metal and sulfides. The Astrophysical Journal 617, 650-655.

Bradley, J.P. (2004) Interplanetary dust particles. In Treatise on Geochemistry (eds. A.M. Davis, H.D. Holland and K.K. Turekian) Elsevier, Amsterdam, Vol. 1, pp. 689-

711, 2004.

Bradley J., Dai Z.R., Erni R., Browning N., Graham G., Weber P., Smith J., Hutcheon I., Ishii H., Bajt S., Floss C., Stadermann F. and Sandford S. (2005) The astronomical 2175 angstrom feature in interplanetary dust particles. Science 307, 244-247. UCRL-JRNL-205703.

Brownlee D.E., Hörz F., Newburn R.L., Zolensky M., Duxbury T.C., Sandford S., Sekanina Z., Tsou P., Hanner M.S., Clark B.C., Green S.F., and Kissel J. 2004. Surface of a young Jupiter family comet 81P/Wild-2: View from the Stardust spacecraft. Science 304: 1764-1769.

Committee on Smaller Facilities, Solid State Sciences Committee, Board on Physics and Astronomy, Division on Engineering and Physical Sciences - National Research Council of the National Academies. (2005) Midsize Facilities: The Infrastructure for Materials Research. The National Academies Press, Washington D.C. www.nap.edu.

Floss C., Stadermann F. J., Bradley J., Dai Z.R., Bajt S. and Graham G. A. (2004) Carbon and nitrogen isotopic anomalies in an anhydrous interplanetary dust particle. Science 303, 1355-1358. UCRL-JRNL-201533. 
Graham G.A., Grant P.G., Chater R.J., Westphal A.J., Kearsley A.T., Snead C., Dominguez G., Butterworth A.L., McPhail D.S., Bench G. and Bradley J.P. (2004) Investigation of ion beam techniques for the in situ analysis and exposure of particles encapsulated by silica aerogel: applicability for Stardust. Meteoritics and Planetary Science. 39, 1461-1474. UCRL-JC-155390.

Graham G.A., Bernas M., Dai Z.R., Bradley J.P., Smith J.B., Weber P.K., Ishii H., Hutcheon I.D. (2005) Recovery of isotopic hotspot in interplanetary dust particles by focused ion beam microscopy: Bridging the gap between NanoSIMS and (S)TEM. Microsc. Microanal. 11(suppl. 2), 1390-1392. UCRL-ABS-21065

Hörz, F., Zolensky, M.E., Bernhard, R.P., See, T.H., Warren, J.L. 2000. Impact features and projectile residues in aerogel exposed on Mir. Icarus 147, 559-579.

Ishii H.A., Graham G.A., Kearsley A.T., Grant P.G., Snead C.J. and Bradley J.P. (2005a) Rapid extraction of dust impact tracks from silica aerogel by ultrasonic micro-blades. Meteorit. Planet. Sci., 40, 1741-1747. UCRL-JRNL-209950.

Ishii H.A., Brennan S., Luening K., Pianetta P., Bradley J.P., Snead C.J. and Westphal A.J. (2005b) Hard $x$-ray spectro-microscopy techniques at SSRL for astromaterials analysis. 36th Lunar and Planetary Science Conference (League City, TX, March 2005), CD-ROM: Abstract 1393. UCRL-ABS-208887.

Westphal, A.J. and Bradley, J.P. (2004) Formation of glass embedded metal and sulfides from shock-accelerated crystalline dust in superbubbles. The Astrophysical Journal 617, 1131-1141. 


\section{Reprints of Selected Papers cited in Results Section}

Ishii H.A., Graham G.A., Kearsley A.T., Grant P.G., Snead C.J. and Bradley J.P. (2005) Rapid extraction of dust impact tracks from silica aerogel by ultrasonic microblades. Meteorit. Planet. Sci., 40, 1741-1747. UCRL-JRNL-209950.

Graham G.A., Grant P.G., Chater R.J., Westphal A.J., Kearsley A.T., Snead C., Dominguez G., Butterworth A.L., McPhail D.S., Bench G. and Bradley J.P. (2004) Investigation of ion beam techniques for the in situ analysis and exposure of particles encapsulated by silica aerogel: applicability for Stardust. Meteoritics and Planetary Science. 39, 1461-1474. UCRL-JC-155390.

Ishii H.A., Brennan S., Luening K., Pianetta P., Bradley J.P., Snead C.J. and Westphal A.J. (2005) Hard x-ray spectro-microscopy techniques at SSRL for astromaterials analysis. 36th Lunar and Planetary Science Conference (League City, TX, March 2005), CD-ROM: Abstract 1393. UCRL-ABS-208887.

Graham G.A., Bernas M., Dai Z.R., Bradley J.P., Smith J.B., Weber P.K., Ishii H., Hutcheon I.D. (2005) Recovery of isotopic hotspot in interplanetary dust particles by focused ion beam microscopy: Bridging the gap between NanoSIMS and (S)TEM. Microsc. Microanal. 11(suppl. 2), 1390-1392. UCRL-ABS-21065.

Floss C., Stadermann F. J., Bradley J., Dai Z.R., Bajt S. and Graham G. A. (2004) Carbon and nitrogen isotopic anomalies in an anhydrous interplanetary dust particle. Science 303, 1355-1358. UCRL-JRNL-201533.

Bradley J., Dai Z.R., Erni R., Browning N., Graham G., Weber P., Smith J., Hutcheon I., Ishii H., Bajt S., Floss C., Stadermann F. and Sandford S. (2005) The astronomical 2175 angstrom feature in interplanetary dust particles. Science 307, 244-247. UCRL-JRNL-205703. 
Ishii H.A., Graham G.A., Kearsley A.T., Grant P.G., Snead C.J. and Bradley J.P. (2005) Rapid extraction of dust impact tracks from silica aerogel by ultrasonic micro-blades. Meteorit. Planet. Sci., 40, 1741-1747. UCRL-JRNL-209950. 
Meteoritics \& Planetary Science 40, Nr 11, 1741-1747 (2005)

Abstract available online at http://meteoritics.org

\title{
Report
}

\section{Rapid extraction of dust impact tracks from silica aerogel by ultrasonic microblades}

\author{
H. A. ISHII ${ }^{1 \dagger *}$, G. A. GRAHAM ${ }^{1 \dagger}$, A. T. KEARSLEY ${ }^{2}$, P. G. GRANT ${ }^{3 \dagger}$, C. J. SNEAD ${ }^{4 \dagger}$, and J. P. BRADLEY ${ }^{1 \dagger}$ \\ ${ }^{1}$ Institute for Geophysics and Planetary Physics, Lawrence Livermore National Laboratory, Livermore, California 94550, USA \\ ${ }^{2}$ Department of Mineralogy, The Natural History Museum, Cromwell Road, London, UK \\ ${ }^{3}$ Center for Accelerator Mass Spectrometry, Lawrence Livermore National Laboratory, Livermore, California 94550, USA \\ ${ }^{4}$ Space Science Laboratory, University of California at Berkeley, Berkeley, California 94720, USA \\ ${ }^{\dagger}$ Member of the Bay Area Particle Analysis Consortium (BayPAC) \\ *Corresponding author. E-mail: hope.ishii@llnl.gov
}

(Received 25 February 2005; revision accepted 09 August 2005)

\begin{abstract}
In January 2006, NASA’s Stardust mission will return with its valuable cargo of the first cometary dust particles captured at hypervelocity speeds in silica aerogel collectors and brought back to Earth. Aerogel, a proven capture medium, is also a candidate for future sample return missions and low-Earth orbit (LEO) deployments. Critical to the science return of Stardust as well as future missions that will use aerogel is the ability to efficiently extract impacted particles from collector tiles. Researchers will be eager to obtain Stardust samples as quickly as possible; tools for the rapid extraction of particle impact tracks that require little construction, training, or investment would be an attractive asset. To this end, we have experimented with diamond and steel microblades. Applying ultrasonic frequency oscillations to these microblades via a piezo-driven holder produces rapid, clean cuts in the aerogel with minimal damage to the surrounding collector tile. With this approach, intact impact tracks and associated particles in aerogel fragments with low-roughness cut surfaces have been extracted from aerogel tiles flown on NASA's Orbital Debris Collector (ODC) experiment. The smooth surfaces produced during cutting reduce imaging artifacts during analysis by scanning electron microscopy (SEM). Some tracks have been dissected to expose the main cavity for eventual isolation of individual impact debris particles and further analysis using techniques such as transmission electron microscopy (TEM) and nano-secondary ion mass spectrometry (nanoSIMS).
\end{abstract}

\section{INTRODUCTION}

A means of efficient extraction of particles from silica aerogel collectors is necessary for the science return of Stardust. This NASA sample return mission, the first from a comet, returns to Earth in early 2006 with its valuable cargo of comet dust that was captured at hypervelocity speeds in aerogel during passage through the coma of Comet Wild-2 (Brownlee et al. 2003; Tuzzolino et al. 2004). In addition to Stardust, aerogel is a likely collector for future sample return missions and is used for capture of hypervelocity ejecta in high-power laser experiments of interest to the Lawrence Livermore National Laboratory (Tobin et al. 2003). Researchers will be eager to obtain Stardust and other aerogel-captured samples for study as quickly as possible, so simple, rapid extraction tools that require little training and have low construction costs would be an attractive asset. Since particles at the termini of impact tracks are expected to be the least altered by impact processes, there is considerable interest in means of extracting individual particles for detailed analysis. In addition, it is important to study intact impact tracks together with their accompanying terminal particles to address the questions of how material is distributed along the impact track and how representative the terminal particle is of the original particle prior to hypervelocity impact. One method for extracting intact cosmic dust impact tracks from silica aerogel tiles is a mature procedure involving sequential perforation of the aerogel with glass needles on computercontrolled micromanipulators (Westphal et al. 2002, 2004). This method is highly successful at removing well-defined aerogel fragments of reasonable optical clarity without causing damage to the surrounding aerogel collector tile. In 
an attempt to speed up and simplify the process of impact track extraction and eventual isolation of terminal particles for Stardust, we have experimented with microblades. Our ultimate goal is a rapid extraction system in a clean electronbeam environment, such as a scanning electron microscope (SEM) or dual-beam focused ion beam system (FIB), for in situ sample preparation, mounting, and analysis.

We have found that piezo-driven ultrasonic frequency (U/S) oscillations applied to very sharp, thin blades generate rapid cuts of unprecedented smoothness with minimal damage to surrounding aerogel. With this ultrasonic microblade extraction technique, still under development, we have extracted several impact tracks from aerogel tiles from NASA's Orbital Debris Collector (ODC) experiment (Hörz et al. 2000) exposed on the Mir Space Station to study the particle environment in low-Earth orbit. Additional tracks were dissected into two halves exposing the main track cavity. Complete or dissected tracks can be extracted in less than an hour. Due to the smooth cut surfaces, impact debris can be readily located and analyzed in situ in the extracted aerogel fragment by SEM. Particles can then be isolated for in-depth study by techniques not amenable to in situ analysis in aerogel, such as transmission electron microscopy (TEM) and secondary ion mass spectrometry (SIMS).

\section{EXTRACTION EQUIPMENT}

We experimented with a range of blades from simple surgical scalpel blades to laser-cut diamond blades. All blades were mounted in a commercially available piezo-driven holder (Eppendorf MicroDissector) on a 3-axis micromanipulator (Eppendorf TransferMan NK2) that was controlled by hand under a Leica MZ16 stereomicroscope with a long working distance objective (Fig. 1a). The MicroDissector's primary applications are intracytoplasmic sperm injection and microdissection of tissue samples, but its piezo-driven holder, controlled by a foot pedal, produces oscillations also ideal for aerogel cutting. Motion is primarily driven along the long axis of the cutting blade over an available frequency range of approximately $25-60 \mathrm{kHz}$ with maximum amplitude of $1.5 \mu \mathrm{m}$. This results in excitation of higher amplitude, transverse vibrations at the blade tip.

Readily available cutting tools such as scalpels and thin razor blades ( $75 \mu \mathrm{m}$ thick) have already been used to cleave aerogel to extract hundreds of terminal particles from impact tracks (Hörz et al. 2000). Even these less elegant cutting tools cut flight-grade aerogel cleanly with little to no fracturing when combined with piezo-driven U/S oscillations. They do, however, create wide channels in the aerogel and tend to generate tearing at depths beyond a few hundred microns.

To reach greater depths, ultra-thin microblades of highcarbon steel and diamond were developed. Two basic blade types, laser-cut from diamond to very narrow, sharp cutting edges, were designed in collaboration with Norsam Technologies (Hillsboro, Oregon, USA): a utility knife- shaped blade and a chisel-shaped blade (Figs. 1b-d), both $5 \mathrm{~mm}$ long, 25 or $55 \mu \mathrm{m}$ thick at the start of the blade and with $10^{\circ}$ or $20^{\circ}$ cutting edges. High-carbon steel microblades with utility-knife shapes were produced from $100 \mu \mathrm{m}$ thick, breakable razor blades (Electron Microscopy Sciences) by snapping off fragments at $\mathrm{LN}_{2}$ temperatures to prevent plastic deformation at the tip. Breakable razor blade fragments were attached to rods with epoxy for mounting in the piezo-driven holder.

Both blade materials have advantages and disadvantages: high-carbon steel microblades can be made in the lab at low cost (albeit low uniformity of blade shape), while diamond microblades are expensive to purchase. Ultra-thin diamond blades create slightly thinner channels with less damage due to their narrow cutting angle and very smooth cutting edge that result in a lower risk of initiating tears in the aerogel. This enables cuts to be made nearer to the track of interest, and closely spaced impact tracks can be extracted separately. Diamond is optically transparent, allowing continuous observation of the cutting region with less distortion and blurring, and since identical diamond microblades are manufactured, the U/S frequency need not be changed significantly between blades. However, with sustained application of U/S oscillations, diamond microblades show a buildup of compressed silica on the cutting surface, leading to an effective dulling of the blade and increased aerogel tearing. The majority of this buildup can be removed by drawing the blade carefully through styrofoam. Any remaining film can presumably be removed in a dilute HF solution or HF vapor that will not attack the diamond. It is unclear why the highcarbon steel microblades have yet to show this problem. Since diamond is brittle, the fine cutting edges and tip also tend to chip, but with care, diamond blades retain their sharp cutting edges longer than steel blades. The microblade extractions presented here have been made with diamond blades for narrow cutting channels and the cleanest cut surfaces.

\section{AEROGEL CUTTING}

Silica aerogel is a highly porous, amorphous glass formed by sol-gel processing followed by supercritical drying. Despite its high strength-to-weight ratio and compressibility, aerogel exhibits glass-like mechanical behavior in tension including spalling and brittle fracture (see, for example, Woignier et al. 2000). These mechanical properties make it challenging to handle and cut without catastrophic failure.

The application of vibrations to blades to facilitate cutting is well-established in nature: leaf-cutting ants, anchored on their hind legs, vibrate their mandibles at $\mathrm{kHz}$ frequencies during leaf cutting (Tautz et al. 1995). Analogous vibrating blade technology (Woods et al. 1994) is used for microtoming tissue sections without freezing or embedding. The U/S oscillations applied to the microblades by the piezodriven holder break up and compress the aerogel locally 

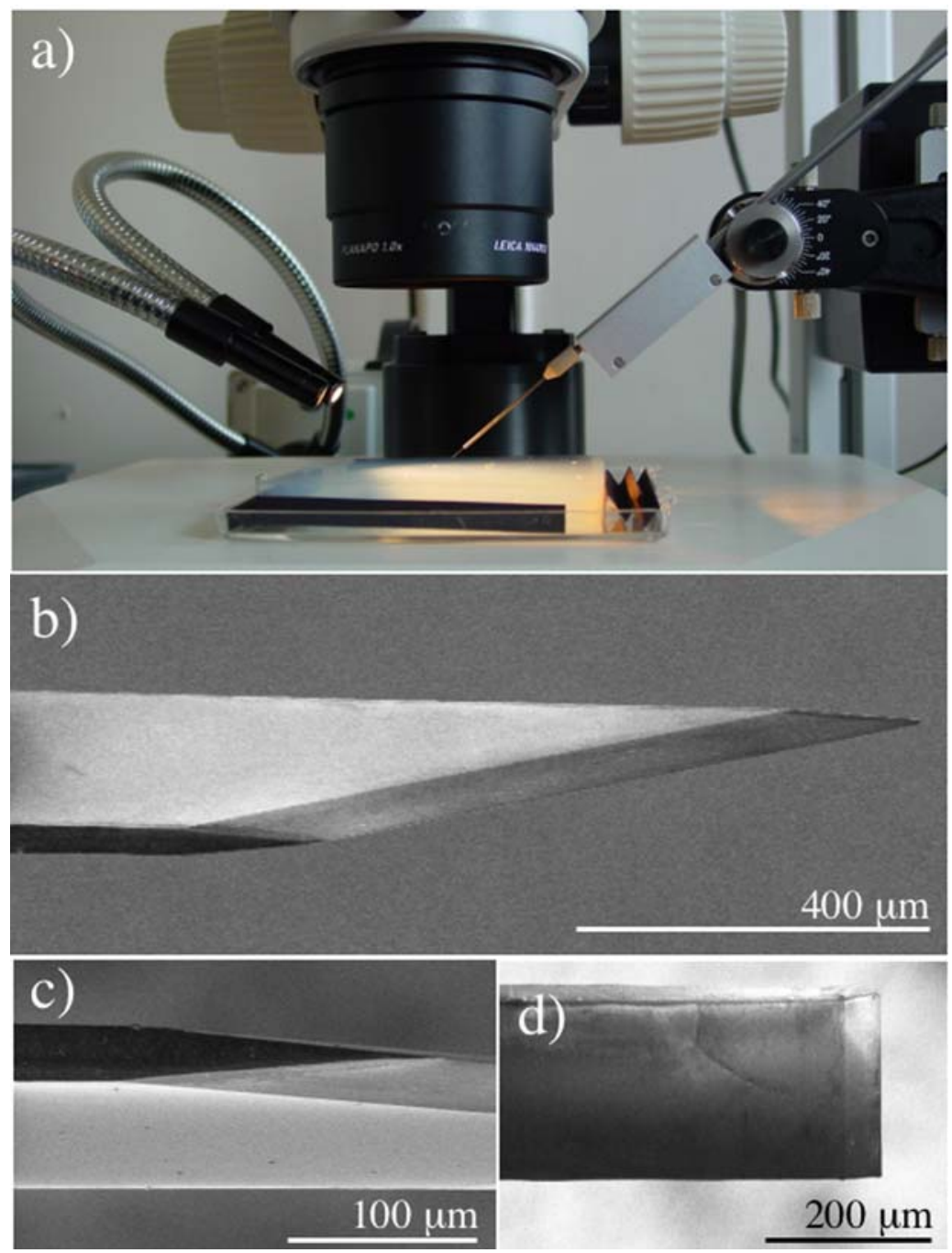

Fig. 1. Equipment for ultrasonic microblade extractions. a) Above an aerogel tile is a diamond microblade mounted in a piezo-driven holder (Eppendorf's MicroDissector) on a micromanipulator under a stereomicroscope with a long working distance objective. b) A secondary electron (SE) image of a diamond utility knife used for vertical cuts. c) An SE image showing the start of the blade's cutting edge. d) An SE image of a diamond chisel used for undercuts (with some artifacts due to charging).

creating a narrow channel that yields a lower friction interaction as the blade passes through. Figure 2a shows cuts made in a flight-grade $\left(20 \mathrm{mg} / \mathrm{cm}^{3}\right)$ silica aerogel blank with and without the piezo-driven U/S oscillations. We have found that U/S frequencies between $30-45 \mathrm{kHz}$, amplitudes of $\sim 1.5$ $\mu \mathrm{m}(100 \%)$ and cutting speeds of $\sim 150-200 \mu \mathrm{m} / \mathrm{sec}$ are optimal for clean aerogel cutting with both diamond and steel microblades. In our experience, lower density silica aerogel (10 mg/cc aerogel and Stardust flight spare) can be cut cleanly at higher speeds with less tendency to tear and spall. The U/S frequency is tuned for each blade via test cuts on a pristine aerogel blank. The degree of aerogel damage created by the cuts is highly sensitive to frequency, as illustrated in Fig. $2 \mathrm{~b}$. Previous attempts to apply this approach to aerogel have been less successful possibly due to non-optimum frequency or amplitude of U/S oscillations. Blade alignment with the micromanipulator axes of motion is readily achieved by placing a mirror below the blade and adjusting the blade rotation while observing the reflection under a microscope.

Two techniques for U/S piezo-assisted cutting of aerogel with microblades have been developed. The first technique involves slowly pushing a vibrating blade directly into the aerogel to the desired depth. By rotating the micromanipulator on its stand, the chisel-style diamond blade has been twice used in this manner to create an undercut in the aerogel below the impact track for the extracted track in Fig. 3c as illustrated by cuts 1 and 2 in Fig. 3a. The same technique was used for the undercut of the extracted track in Fig. 3d.

The second technique is for vertical cuts, The utility knife-style blades (diamond or steel) are drawn with slow motions parallel to the surface. After each cut, the blade is lifted above the aerogel surface to return to the starting position, and the next cutting pass is made $\sim 50 \mu \mathrm{m}$ deeper. 

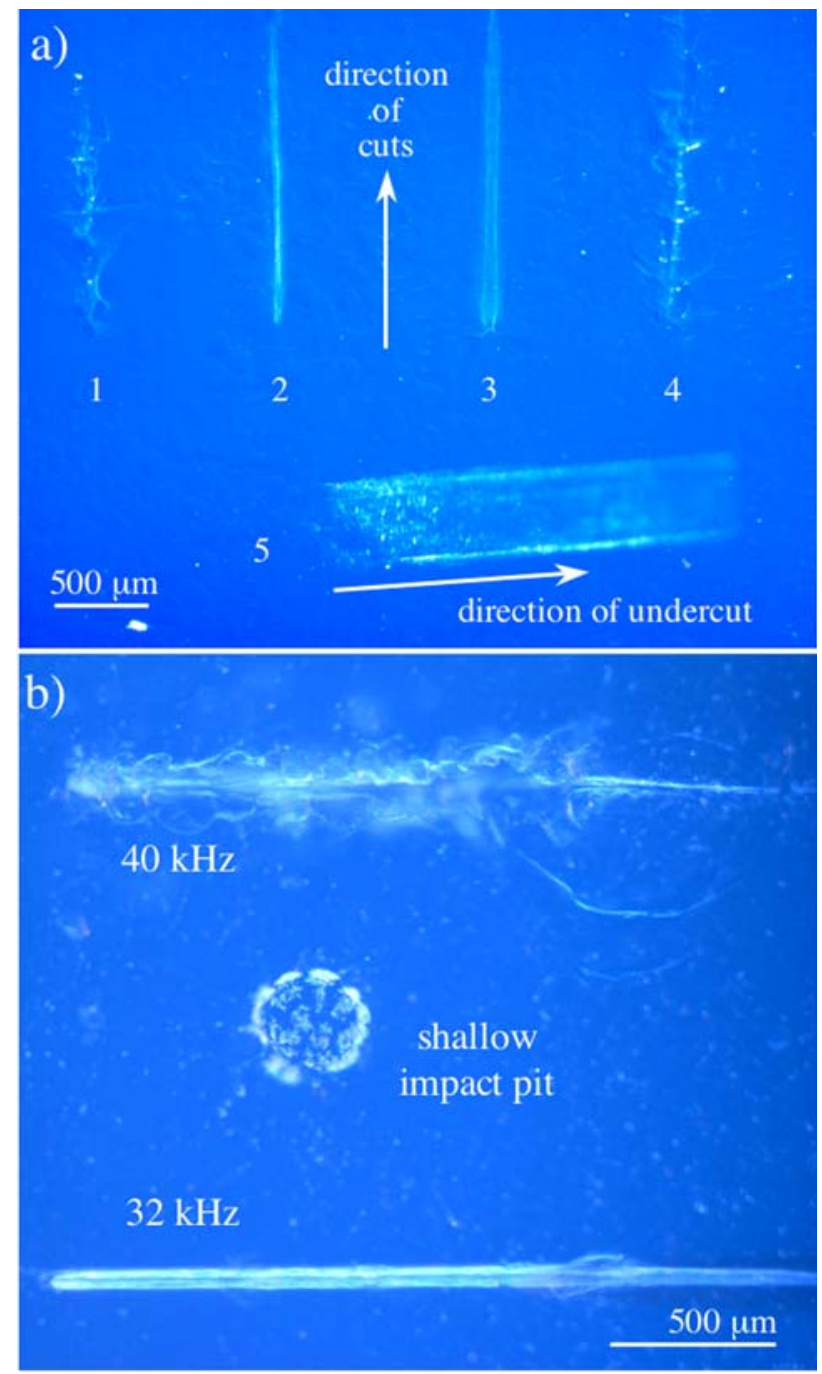

Fig. 2. A demonstration of the effectiveness of aerogel cutting with ultrasonic (U/S) microblades. a) An optical image of test cuts on flight grade aerogel $\left(20 \mathrm{mg} / \mathrm{cm}^{3}\right)$. Cuts 1 and 4 (200 $\mu \mathrm{m}$ deep) were made without piezo-driven U/S oscillations, and cuts 2 , 3 , and 5 were made with U/S oscillations. Cuts 1 and 2 were made by a diamond utility knife, cuts 3 and 4 were made by the steel blade, and the undercut, cut 5 , was made by a diamond chisel at an angle of $25^{\circ}$ to the aerogel surface. b) The frequency sensitivity of microblade cutting. Cuts on either side of a shallow impact pit feature in ODC tile 2D04 were made with U/S frequencies of $40 \mathrm{kHz}$ (upper cut) and $32 \mathrm{kHz}$ (lower cut). Both were made using an ultra-thin diamond utility knife to a depth of $1.4 \mathrm{~mm}$.

The aerogel is rotated for subsequent cuts. Cuts 3, 4, and 5 in Fig. 3b were made in this way. This second approach yields highly smooth cut surfaces.

It should be noted that damage (due to handling, for example) can create a thin surface crust of higher density aerogel that results in fragmentation of the surface rather than disintegration. This aerogel debris falls into the channel and is tumbled by the blade, creating ragged edges. For cuts sufficiently far from the track $(\sim 200-300 \mu \mathrm{m})$, the track itself is unharmed. This problem has not been found on aerogel tiles
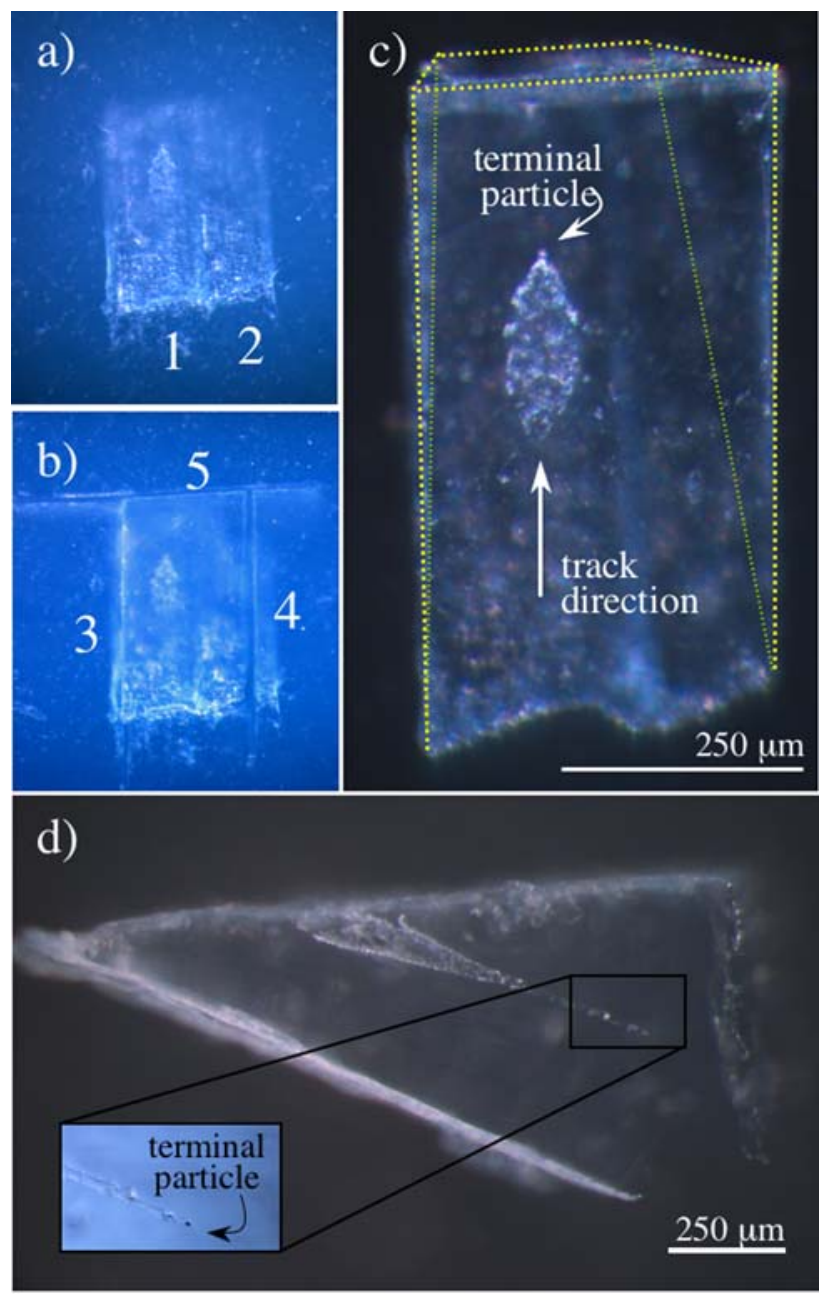

Fig. 3. Optical images of the extraction of ODC impact tracks from tile 2C01 using U/S piezo-driven oscillating diamond microblades. a) The first cuts (1 and 2) are undercuts made with a diamond chisel at an oblique angle to the aerogel surface and extending below the impact track. b) The final three cuts (3, 4, and 5) are vertical cuts made with a diamond utility knife perpendicular to the aerogel surface. c) The aerogel fragment containing the impact track after removal from the aerogel collector tile. The dashed yellow lines indicate the aerogel boundaries. d) A side view of another extracted aerogel wedge containing an impact track from ODC tile 2C01. The top surface shows some pre-existing damage. Inset: The terminal particle at the end of the stylus in transmitted light.

flown as part of the ODC experiment but having little handling damage.

U/S piezo-assisted dissection can be used to cleave the main cavity of large impact tracks in order to expose particulates and ablated material in the track walls. This results in the terminal particles residing closer to the surface simplifying their eventual isolation from the aerogel. These dissections can be carried out in the bulk aerogel collector tile. One half of the impact track can be extracted, leaving the remaining half in the tile (as was done with the extraction shown in Fig. 4b), or both halves can be extracted. Because the impact track itself is "damage" in the aerogel, there is 

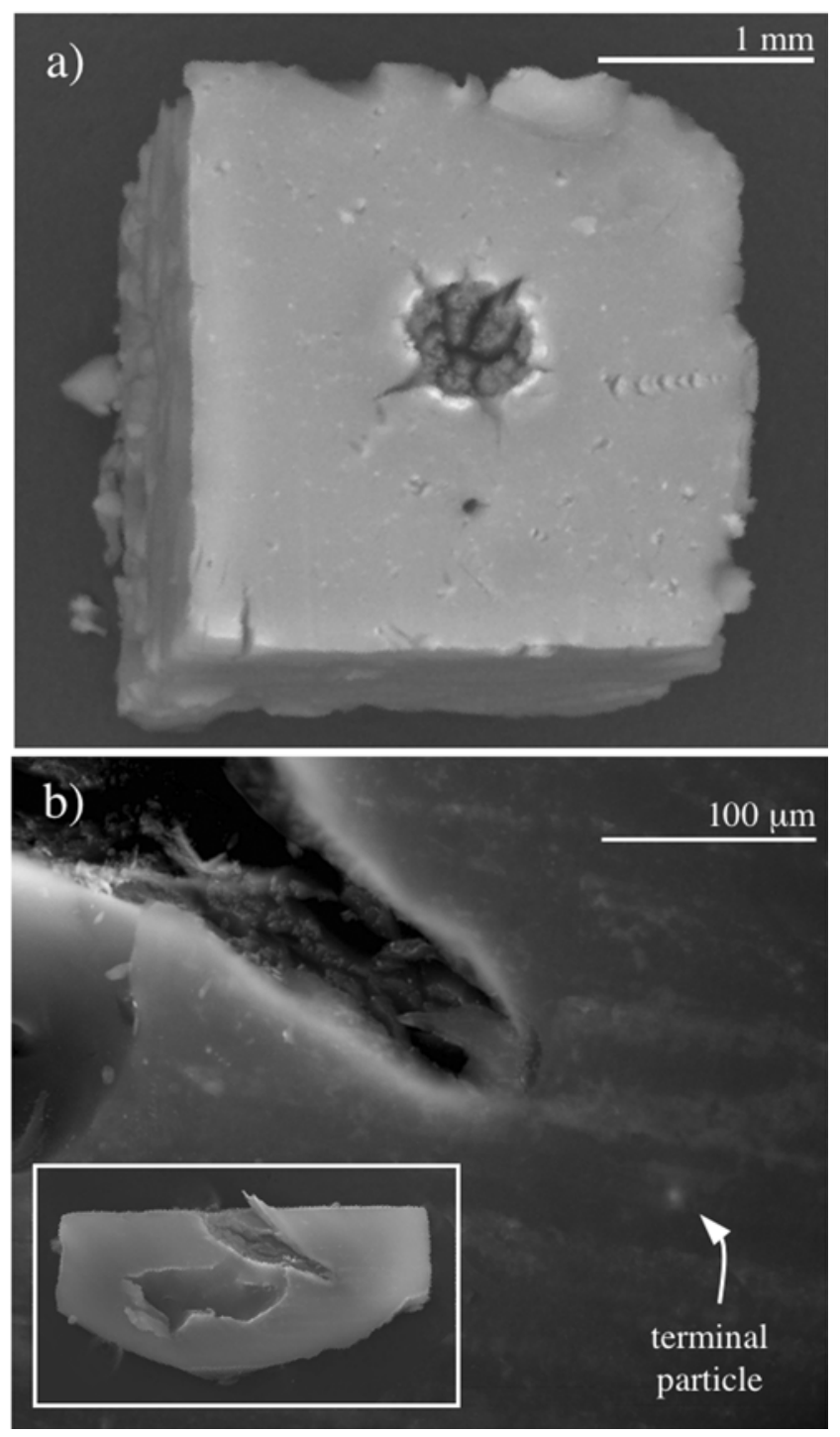

Fig. 4. Smooth cut surfaces produced by U/S microblades reduce imaging artifacts and allow quick identification of impact particles. a) Backscattered electron (BSE) image of the final extracted aerogel cube containing the shallow impact pit feature from Fig. 2b. The downward-facing surface was cut at the optimized U/S frequency for the diamond microblade. b) BSE image of an ODC track (terminal particle visible just sub-surface as a diffuse bright spot) from tile 1F04 dissected using a diamond utility knife with piezo-driven U/S oscillations. Inset: Secondary electron image of the entire dissected aerogel fragment. Tearing below the track propagated from the preexisting damage at the bottom surface of the impact track.

some risk of further propagation of existing cracks, as seen in the inset of Fig. 4b. These cracks tend to propagate in the direction of blade motion at acute angles. This is especially an issue in large impact tracks; however, slow and controlled blade motion minimizes the extent of aerogel tearing. In addition, there is some compression of the aerogel at the edges of the cut as well as some saw-kerf (material loss due to breakup or sticking on the diamond blade). For small tracks ( $<50 \mu \mathrm{m}$ diameter), there is a risk of eroding away much of
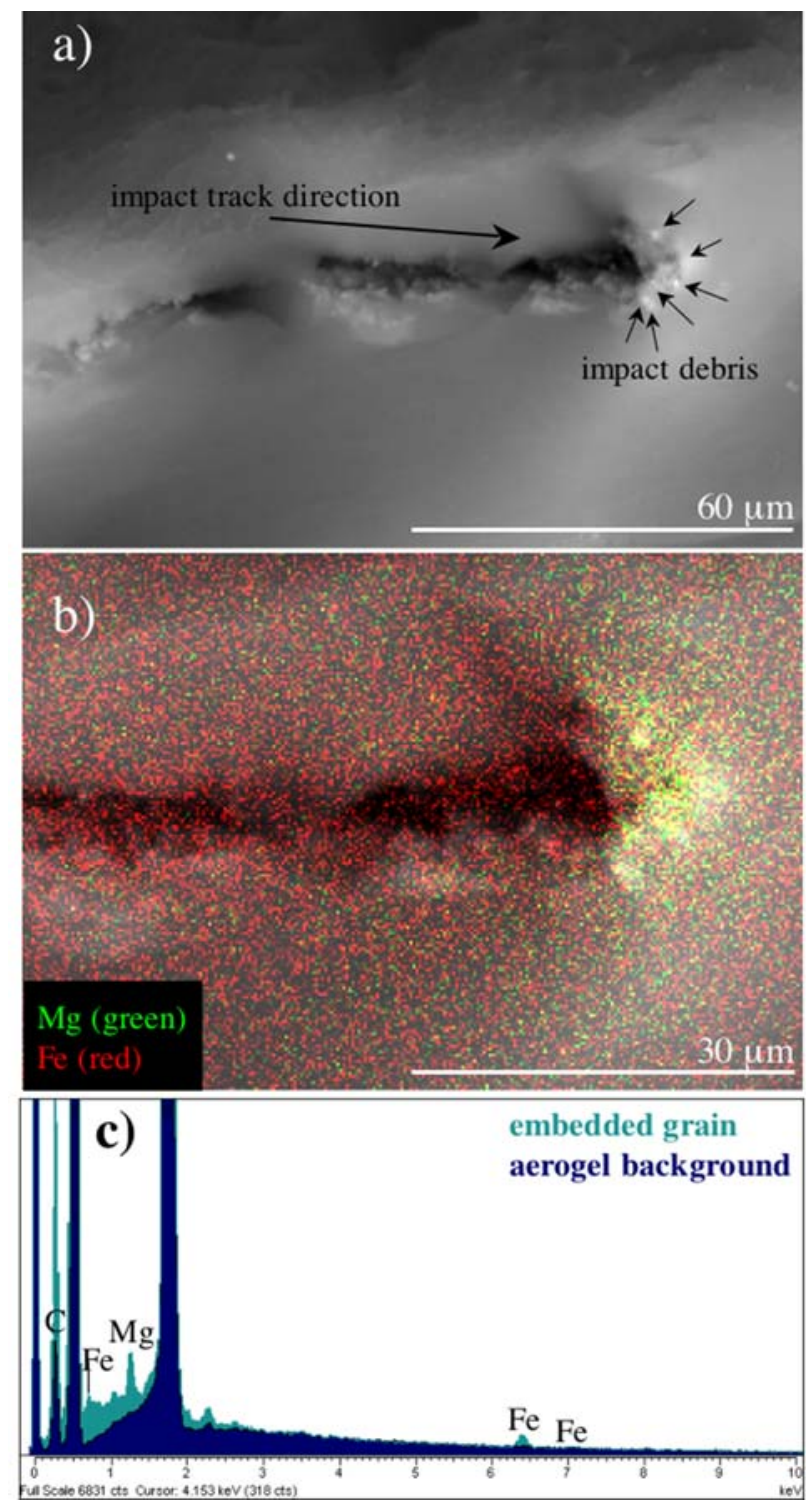

Fig. 5. a) A backscattered electron (BSE) image of an aerogel fragment extracted from ODC collector tile 2C01. This track was extracted whole from the tile using U/S microblades and then dissected out-of-tile by a diamond blade without U/S oscillations to expose a region of the track's main cavity. Several bright impact debris particles at the end of the exposed region are identified by small arrows. The intensity from the particles appears diffuse due to the fact that they are still beneath the surface of the aerogel. b) The high intensity locations (yellow-white) in X-ray energy-dispersive maps for Mg (green) and Fe (red) correlate strongly with the bright particles in the BSE image. c) A typical energy-dispersive spectrum for one of the individual particles is consistent with a silicate composition.

the material of interest. In this case, tracks can be extracted whole from the aerogel tile with U/S piezo assistance and then cleaved out-of-tile using a sharp blade without U/S piezo oscillations, as in Fig. 5a. This results in a less predictable and less flat dissection surface, but the fracture surface is still very smooth. 


\section{PRELIMINARY ANALYSIS OF EXTRACTED TRACKS}

An end goal for U/S microblade development is incorporation of the blades in an SEM or dual-beam FIB environment to enable sample recovery/preparation and preliminary characterization. This goal is motivated by previous analysis of extraterrestrial material preserved in ODC impact tracks that shows a high degree of fragmentation of the original projectile with debris deposited down the length of the track (e.g., Borg et al. 2004). This underscores the need for a means of identifying and removing particles of various sizes.

For the SEM studies, aerogel fragments containing dissected impact tracks and one aerogel fragment containing a shallow impact pit, all extracted using the U/S microblade technique, were mounted on standard SEM mounts with adhesive, conductive carbon pads and examined in a LEO 1455VP SEM fitted with an Oxford INCA Pentafet energy dispersive X-ray spectrometer (EDS). A $15 \mathrm{~mm}$ working distance and a $20 \mathrm{kV}$ accelerating voltage were used for both imaging and microanalysis. A $700 \mathrm{pA}$ beam current was used for imaging and a $1.2 \mathrm{nA}$ beam current was used for microanalysis.

\section{Imaging}

Typically, secondary electron imaging offers only topographic information while backscattered electron (BSE) imaging provides atomic number contrast. Thus, BSE imaging is an obvious candidate for locating higher density micrometeoroid fragments in dissected impact tracks in lowdensity silica aerogel. However, there are a number of issues in the track alone: There is major beam dispersion and electron energy loss with depth in the aerogel, and much of the debris is located subsurface. In addition, surface roughness and densified aerogel formed by compression and by melting and recondensation in the track add noise to the image. In the past, it has been difficult to distinguish micrometeoroid fragments from the surrounding aerogel by BSE imaging of tracks extracted using the glass needle extraction method. Compositional contrast has been largely obscured by image artifacts generated by additional high roughness and compacted aerogel debris produced by the glass needle extraction method on the cut surfaces surrounding the track. This roughness and additional debris add more artifacts to the already complex picture in the track cavity. In contrast, the U/S microblade extraction method generates very smooth surface cuts that reduce imaging artifacts in both secondary and backscattered electron imaging. The low-roughness surface removes uncertainties in identifying impact debris so that particles exposed on the surface, or even located subsurface, can be quickly located by BSE imaging. The ease of locating cosmic dust particles within smooth-cut extracted tracks will simplify in situ recovery of isolated particles in a SEM or dual-beam FIB (Graham et al. 2004; Graham et al. 2005). Figure 4a contains a BSE image of the shallow impact pit cut from a bulk ODC aerogel tile (Fig. 2b). The downward-facing surface shows the smooth-cut surface produced by U/S microblades at the optimized frequency. Figure $4 \mathrm{~b}$ shows the BSE image of an ODC impact track dissected using a diamond utility knife with piezo-driven U/S oscillations. The terminal particle lies just below the aerogel surface and appears as a clearly distinguishable, bright spot in the BSE image. The bright spot has a diffuse appearance due to the subsurface location of the particle.

\section{Microanalysis}

All of the dust particles captured in aerogel, from either LEO collectors or the Stardust collector, have experienced hypervelocity impact. As a result, they are likely to have undergone some degree of alteration, most likely fragmentation and thermal modification, and therefore, they are not pristine in nature (Anderson and Ahrens 1994; Hörz et al. 2000). To ensure the data acquired is representative of the composition of a cometary grain prior to hypervelocity capture, a number of particulates associated with a single impact should be characterized. While other techniques, such as synchrotron X-ray fluorescence (e.g., Borg et al. 2004), have been shown capable of such studies, EDS mapping and single spot analysis are also well-suited to identifying candidates for isolation as "naked" particles for in-depth TEM or SIMS analysis. Figure 5a is a BSE image of an impact track associated with the chondritic swarm event previously identified in the ODC tiles (Hörz et al. 2000). This track was extracted whole from the bulk aerogel tile and then dissected to expose the main cavity by a diamond blade without applying U/S oscillations. The cleaved surface again has very low roughness, allowing imaging of impact debris that appears as a cluster of bright spots in the BSE image. EDS mapping in Fig. 5b shows elevated levels of $\mathrm{Mg}$ and $\mathrm{Fe}$ in the region containing the particulates, which is confirmed by single spot analyses (Fig. 5c). While it is not possible to unambiguously identify the mineralogy of the particulates due to the high background from the surrounding aerogel, it is highly probable that they are fragments of an Mg-Fe silicate. For rigorous mineralogical analysis, isolation of the particulates from the silica aerogel fragment is necessary.

\section{SUMMARY AND FUTURE WORK}

We have presented a simple, rapid, and accessible method for the extraction of hypervelocity impact tracks from silica aerogel tiles. We demonstrated that ultrasonic frequency oscillations applied to thin, sharp microblades 
quickly produce clean cuts in aerogel with minimal damage to the surrounding tile. Extraction time depends on the details of track depth and morphology; however, using our current setup under hand control, complete tracks have been extracted in less than an hour. In addition to the optical microscope and micromanipulator normally found in laboratories involved in small particle manipulation, a system for extracting complete or dissected impact tracks from bulk aerogel tiles requires only a piezo-driven holder and microblades. Although piezodriven holders may be home-built, we have chosen a commercially available piezo-driven holder to generate ultrasonic oscillations of the appropriate frequency, amplitude and direction. For the $\sim 25-100 \mu \mathrm{m}$ thick diamond and steel microblades used in this work, frequencies between 30 and 40 $\mathrm{kHz}$ are optimum. With this equipment, impact tracks have been extracted from aerogel tiles flown on NASA's Orbital Debris Collector experiment. Tracks have also been dissected exposing the main cavity for SEM imaging and elemental analysis. The smoothness of the resulting cut surfaces allows quick identification of impact debris particles for EDS analysis and, if desired, eventual isolation of individual particles for other analyses.

The various methods now available for extracting impact tracks/pits and terminal particles/residues from silica aerogel offer advantages in different conditions. For large tracks or pits that are sufficiently widely spaced, cleaving the aerogel via handheld razor blades may provide the least expensive and quickest method of track and/or particle extraction. The ultrasonic microblades presented here are well-suited to small and intermediate-size features that may be more closely spaced. The computer-controlled glass needle extraction method is best applied to small and very closely spaced tracks since the technique, although slower and more expensive in setup cost, is not limited to straight cuts and causes very little damage outside the extracted region.

Our current micromanipulator has orthogonal motions, one perpendicular to the sample surface. The entire unit can be rotated to make undercuts in aerogel not perpendicular to the sample surface. We are installing a micromanipulator with an additional axis for angled blade motions. This will permit freeing an aerogel fragment completely from a tile without adjusting micromanipulator angle. For tracks that are nearly perpendicular to the surface, such as those expected in the Stardust aerogel, an inverted regular pyramid can be extracted with 3 or 4 angled cuts. For tracks at more oblique angles, one or more of those cuts may be perpendicular to the surface. To assist in such extractions, modified chisel-style diamond microblades have been designed and will be tested.

Acknowledgments-Portions of this work were performed under the auspices of the Department of Energy by the University of California, Lawrence Livermore National Laboratory under Contract No. W-7405-Eng-48. This research is also supported by NASA Grants NNH04AB49I and NAG5-11902. S. M. Brennan is thanked for suggesting ultra-thin blades. We also thank F. Hörz for his constructive review of the manuscript.

\section{Editorial Handling—Dr. Donald Brownlee}

\section{REFERENCES}

Anderson W. W. and Ahrens T. J. 1994. Physics of interplanetary dust capture via impact into organic polymer foams. Journal of Geophysical Research E99:2063-2071.

Borg J., Djouadi Z., Matrajt G., Martinez-Criado G., Snead C. J., Somogyi A., and Westphal A. J. 2004. In-situ analyses of Earth orbital grains trapped in aerogel using synchrotron $\mathrm{x}$-ray microfluorescence techniques (abstract \#1580). 35th Lunar and Planetary Science Conference. CD-ROM.

Brownlee D. E., Tsou P., Anderson J. D., Hanner M. S., Newburn R. L., Sekanina Z., Clark B. C., Hörz F., Zolensky M. E., Kissel J., McDonnell J. A. M., Sandford S. A., and Tuzzolino A. J. 2003. Stardust: Comet and interstellar dust sample return mission. Journal of Geophysical Research 108:1-1-1-15.

Graham G. A., Bradley J. P., Bernas M., Stroud R. M., Dai Z. R., Floss C., Stadermann F. J., Snead C. J., and Westphal A. J. 2004. Focused ion beam recovery and analysis of interplanetary dust particles (IDPs) and Stardust analogues (abstract \#2044). 35th Lunar and Planetary Science Conference. CD-ROM.

Graham G. A, Sheffield-Parker J., Bradley J. P., Kearsley A. T., Dai Z. R., Mayo S. C., Teslich N., Snead C., Westphal A. J., and Ishii H. 2005. Electron beam analysis of micrometeoroids captured in aerogel as Stardust analogues (abstract \#2078). 36th Lunar and Planetary Science Conference. CD-ROM.

Hörz F., Zolensky M. E., Bernhard R. P., See T. H., and Warren J. L. 2000. Impact features and projectile residues in aerogel exposed on Mir. Icarus 147:559-579.

Tautz J., Roces F., and Hölldobler B. 1995. Use of a sound-based vibratome by leaf-cutting ants. Science 267:84-87.

Tobin M., Andrew J., Haupt D., Mann K., Poco J. F., Satcher J. H. Jr., Curran D., Tokheim R., and Eder D. 2003. Using silica aerogel to characterize hypervelocity shrapnel produced in high power laser experiments. International Journal of Impact Engineering 29:713-721.

Tuzzolino A. J., Economou T. E., Clark B. C., Tsou P., Brownlee D. E., Green S. F., McDonnell J. A. M., McBride N., and Colwell M. T. S. 2004 Dust measurements in the coma of Comet 81P/ Wild 2 by the Dust Flux Monitor Instrument. Science 304:17761780.

Westphal A. J., Snead C., Borg J., Quirico E., Raynal P., Zolensky M., Ferrini G., Colangeli L., and Palumbo P. 2002. Small hypervelocity particles captured in aerogel collectors: Location, extraction, handling and storage. Meteoritics \& Planetary Science 37:855-865.

Westphal A. J., Snead C., Butterworth A., Graham G. A., Bradley J. P., Bajt S., Grant P. G., Bench G., Brennan S., and Pianetta P. 2004. Aerogel keystones: Extraction of complete hypervelocity impact events from aerogel collectors. Meteoritics \& Planetary Science 39:1375-1386.

Woignier T., Despetis F., Alaoui A., Etienne P., and Phalippou J. 2000. Mechanical properties of gel-derived materials. Journal of Sol-Gel Science and Technology 19:163-169.

Woods A. W. and Ellis R. C. 1994. Laboratory histopathology: A complete reference. Edinburgh: Churchill Livingstone. 2 volumes. 
Graham G.A., Grant P.G., Chater R.J., Westphal A.J., Kearsley A.T., Snead C., Dominguez G., Butterworth A.L., McPhail D.S., Bench G. and Bradley J.P. (2004) Investigation of ion beam techniques for the in situ analysis and exposure of particles encapsulated by silica aerogel: applicability for Stardust. Meteoritics and Planetary Science. 39, 1461-1474. UCRL-JC-155390. 


\title{
Investigation of ion beam techniques for the analysis and exposure of particles encapsulated by silica aerogel: Applicability for Stardust
}

\author{
G. A. GRAHAM, ${ }^{1,5^{*} \#}$ P. G. GRANT, ${ }^{2 \#}$ R. J. CHATER,${ }^{3}$ A. J. WESTPHAL, ${ }^{4 \#}$ A. T. KEARSLEY, ${ }^{5}$ C. SNEAD, ${ }^{4 \#}$ \\ G. DOMÍNGUEZ, ${ }^{4 \#}$ A. L. BUTTERWORTH, ${ }^{4 \#}$ D. S. MCPHAIL,${ }^{3}$ G. BENCH, ${ }^{2 \#}$ and J. P. BRADLEY ${ }^{1 \#}$ \\ ${ }^{1}$ Institute of Geophysics and Planetary Physics, Lawrence Livermore National Laboratory, Livermore, California 94551, USA \\ ${ }^{2}$ Center for Accelerator Mass Spectrometry, Lawrence Livermore National Laboratory, Livermore, California 94551, USA \\ ${ }^{3}$ Department of Materials, Imperial College, London, SW7 2AZ, UK \\ ${ }^{4}$ Space Sciences Laboratory, University of California at Berkeley, Berkeley, California 94720, USA \\ ${ }^{5}$ Department of Mineralogy, The Natural History Museum, London, SW7 5BD, UK \\ \#Member of BayPAC (Bay Area Particle Analysis Consortium) \\ *Corresponding author. E-mail: graham42@1lnl.gov \\ (Received 19 September 2003; revision accepted 25 June 2004)
}

\begin{abstract}
In 2006, the Stardust spacecraft will return to Earth with cometary and perhaps interstellar dust particles embedded in silica aerogel collectors for analysis in terrestrial laboratories. These particles will be the first sample return from a solid planetary body since the Apollo missions. In preparation for the return, analogue particles were implanted into a keystone of silica aerogel that had been extracted from bulk silica aerogel using the optical technique described in Westphal et al. (2004). These particles were subsequently analyzed using analytical techniques associated with the use of a nuclear microprobe. The particles have been analyzed using: a) scanning transmission ion microscopy (STIM) that enables quantitative density imaging; b) proton elastic scattering analysis (PESA) and proton backscattering (PBS) for the detection of light elements including hydrogen; and c) proton-induced X-ray emission (PIXE) for elements with $Z>11$. These analytical techniques have enabled us to quantify the composition of the encapsulated particles. A significant observation from the study is the variable column density of the silica aerogel. We also observed organic contamination within the silica aerogel. The implanted particles were then subjected to focused ion beam (FIB) milling using a $30 \mathrm{keV}$ gallium ion beam to ablate silica aerogel in site-specific areas to expose embedded particles. An ion polished flat surface of one of the particles was also prepared using the FIB. Here, we show that ion beam techniques have great potential in assisting with the analysis and exposure of Stardust particles.
\end{abstract}

\section{INTRODUCTION}

Analytical instrument developments over the past 10 years have enabled new levels of mineralogical and chemical investigations of interplanetary dust particles (IDPs) (e.g., Dai et al. 2002; Messenger et al. 2003). Despite the comparisons that can be drawn between the composition of IDPs and astronomical features (e.g., Keller et al. 2002), it is currently not possible to unambiguously identify IDPs with specific parent bodies. The successful capture of near-intact particles using silica aerogel, first in the laboratory and then in low-Earth orbit (Barrett et al. 1992; Brownlee et al. 1994; Tsou 1995), lead to the NASA Discovery class mission Stardust, the first cometary sample return (e.g., Brownlee et al. 1997). In January 2004, the Stardust flyby of comet Wild 2 was successful, and it is expected that a large number of cometary particles were captured in the dedicated silica aerogel collector (Brownlee et al. 2004). The scientific community must now wait until January 2006 when the sample return capsule lands back on Earth to harvest the bounty of cometary particles and contemporary interstellar grains. Instruments already suitable for the analysis of IDPs are also suitable to carry out the analysis of the unique particles captured by Stardust (e.g., Zolensky et al. 2000; Stephan 2001). Despite the availability of instrumentation, there are a number of technical challenges to be resolved before sample return. Perhaps the most significant challenges are technical rather than scientific because the identification, extraction, and handling of small captured hypervelocity particles from silica aerogel is not straightforward (Tsou 


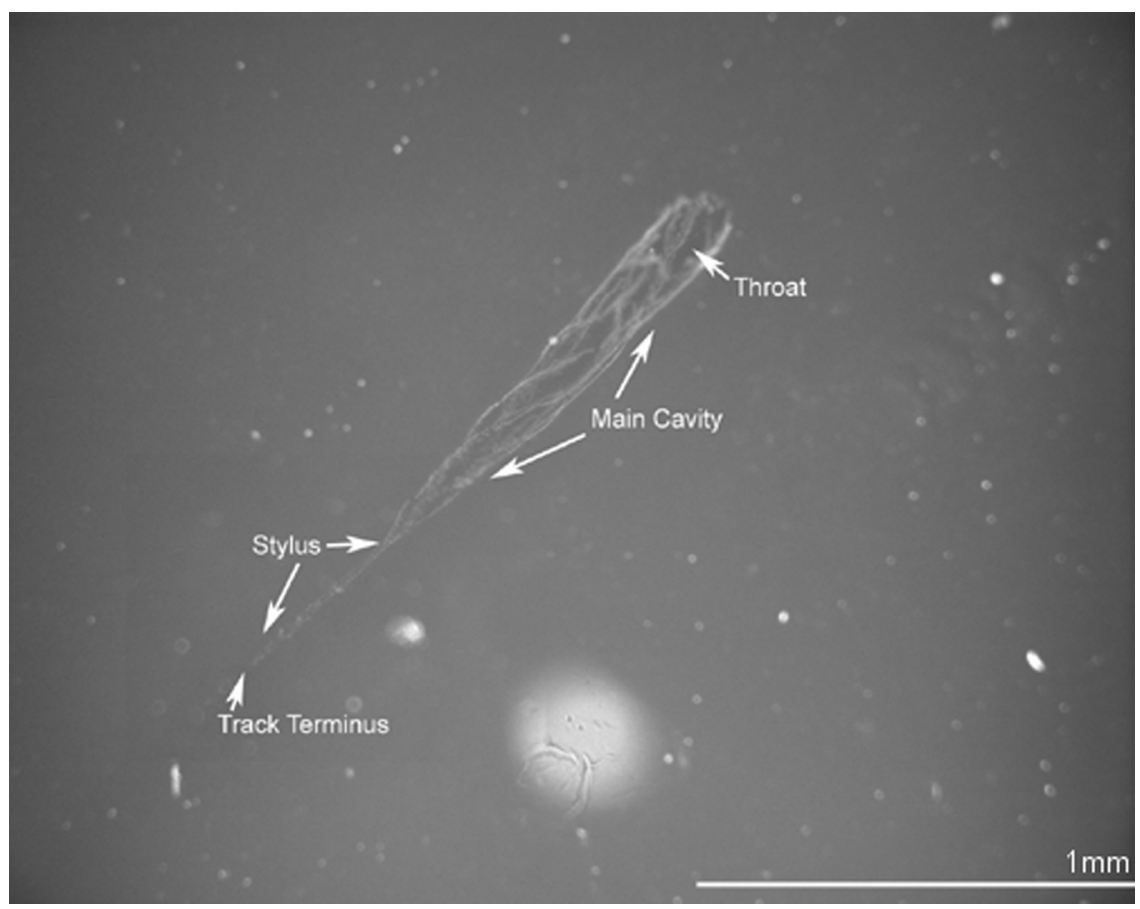

Fig. 1. Optical transmitted light micrograph of a hypervelocity impact track preserved in silica aerogel (density of $\left.0.02 \mathrm{~g} / \mathrm{cm}^{3}\right)$ from the NASA Orbital Debris Collector Experiment. The track terminology is taken from Hörz et al. (2000).

1995). Previous efforts at particulate extraction have included cleaving the aerogel apart using biological dissection razorblades to expose individual particles for extraction with a micromanipulator. Although extremely simple, this technique was used very successfully in the recovery of a number of particles (over 100) from the silica aerogel collector exposed on the Mir space station (Bernhard et al. 2000). However, this technique can lead to a catastrophic failure of the aerogel during the cleaving that results in the loss of particulate material (Stadermann and Floss 2000). Laser ablation extraction has also been used to recover particles accelerated into silica aerogel using a light-gas-gun (Graham et al. 2001). Westphal et al. (2002), using automated micromanipulators and glass micro-needles, extracted individual particles down to $3 \mu \mathrm{m}$ in diameter and, more recently, keystones of silica aerogel containing entire impact tracks (Westphal et al. 2004). Beyond mechanical extractions, it is important to investigate emerging technologies that could be suitable for Stardust. Despite the range of extensive techniques available for small particle analysis (Zolensky et al. 2000), some of these techniques degrade volatile-rich materials, others produce contamination, and others require tailored specimen preparation. Therefore, it is important that application of the various analytical methods be hierarchical. Furthermore, since thousands of cometary particles may be captured, it is essential that analytical techniques capable of rapid and non-destructive in situ analysis be identified and evaluated. Synchrotron-based analytical techniques appear to be promising for making in situ measurements (e.g., Raynal et al. 2000; Flynn et al. 2001, 2003; Borg et al. 2002; Keller et al. 2003). Other small-scale laboratory-based techniques have been successfully applied to embedded particles, e.g., microRaman spectroscopy (Burchell et al. 2001; Kearsley et al. 2001; Graham et al. 2001). In this paper, which is the second in a series of research on extraction and analysis techniques relevant to Stardust carried out by BayPAC (Bay Area Particle Analysis Consortium), a consortium of Lawrence Livermore National Laboratory (LLNL), University of California at Berkeley, Ernest Orlando Lawrence Berkeley National Laboratory, and Stanford University, we discuss the applicability of ion beam techniques for the analysis of material embedded in silica aerogel using: i) a $3 \mathrm{MeV}$ hydrogen ion beam; and ii) $30 \mathrm{keV}$ focused gallium ion beam to expose embedded materials for subsequent detailed microanalysis.

\section{PARTICLE CHARACTERIZATION USING A NUCLEAR MICROPROBE}

The development of the extraction technique described in Westphal et al. $(2002,2004)$ is a significant milestone. However, it is only the first stage of the analytical strategy for Stardust particles. The initial scientific questions to be asked about the preserved particles will be very simple: what is their morphology, mineralogy, and bulk chemistry, and how do they compare to the current repository of IDPs?

When a particle is captured in silica aerogel as a result of a hypervelocity collision, a number of characteristic features 
are generated (see Hörz et al. [2000] and Fig. 1). Silica aerogel experiments (e.g., NASA Orbital Debris Collector Experiment $[\mathrm{ODC}]$ ) in low-Earth orbit (LEO) enable the investigation of "real" micro-particles captured in silica aerogel collectors; however, such impact events will have occurred over a range of velocities (typically $>10 \mathrm{~km} / \mathrm{s}$ ). Therefore, these may not be representative of the features preserved in the Stardust collectors where the encounter velocity was approximately $6 \mathrm{~km} / \mathrm{s}$. The LEO-derived impact events tend to show a high degree of particle fragmentation on the micron and sub-micron scales (e.g., Hörz et al. 2000; Westphal et al. 2004). Therefore, it is reasonable to assume that if cometary particles are similar to fluffy anhydrous IDPs, they may exhibit similar behavior upon collision with the silica aerogel collectors, even at the lower velocity.

If the Stardust silica aerogel collectors return with a number of impact events showing fragmentation of the cometary debris, then one of the critical tasks will be to obtain a true representative composition. Therefore, any preliminary characterization will scan the entire impact event for cometary debris. Ideally, the applied technique will have limited or no detrimental effects on the encapsulated particles. The major and minor elemental compositions of IDPs have been successfully investigated using nuclear microprobe techniques (e.g., Arndt et al. 1996; Wies et al. 2002). However, the techniques have not been widely used in cosmic dust research despite being relatively non-destructive (Zolensky et al. 2000). Thus, nuclear microprobe analysis could be well-suited for characterizing captured particles due to the ability of the primary beam to penetrate the silica aerogel and the adequate signal that can be detected during sample-beam interactions (Morse et al. 1997). These interactions can be detected in a number of ways: a) scanning transmission ion microscopy (STIM) that enables quantitative density imaging; b) proton elastic scattering analysis (PESA) and proton backscattering (PBS) for the detection of light elements including hydrogen; and c) proton-induced X-ray Emission (PIXE) for elements with $\mathrm{Z}>11$.

\section{Sample Preparation}

For the experimental work described here, $20 \mathrm{mg} / \mathrm{cc}$ silica aerogel manufactured at NASA/JPL by Dr. Steve Jones was used. Previous laboratory simulations that mimic the Stardust capture have used light-gas-guns, as these enable the acceleration of the projectiles at the mission encounter velocity of $6.1 \mathrm{~km} / \mathrm{s}$ (e.g., Hörz et al. 1998; Burchell et al. 2001). However, because the initial purpose of the analysis described here was to investigate the suitability of the nuclear microprobe, specially selected particles were chosen that would test the analytical techniques. Therefore, hypervelocity acceleration of materials was not required. A glass microneedle has been used to implant the following materials into the bulk aerogel at approximately $10 \mu \mathrm{m}$ depth: a) a tungsten sphere (10 $\mu \mathrm{m}$ in diameter) because it has extremely high density compared to the silica aerogel and should, therefore, be easily detected using STIM, and since it is a high Z element, it should be easy to map using PIXE; b) a polystyrene sphere ( $7 \mu \mathrm{m}$ in diameter) to evaluate the possibility of detecting organic species during the initial characterization using PESA and PBS; c) a fragment of the matrix material from the Orgueil meteorite to represent a complex unknown meteorite material, as it is possible that Stardust may return particle compositions that, as yet, have been routinely identified in IDPs (Fig. 2). While the manual embedding technique does not allow the investigation of impact effects such as shock and thermal alteration on the particles, it does enable a wide range of particles, including IDPs, to be embedded into silica aerogel. This allows the nuclear microprobe's ability to detect both inorganic and organic material in a wide range of particles to be tested. After implantation, a small wedge or the so-called "keystone" of aerogel containing the analogue particles was extracted from the bulk using the mechanical recovery techniques described in Westphal et al. (2004).

The silicon microforklifts that are used to extract the keystones from bulk silica aerogel have been designed to integrate with a number of instrument mounts including the nuclear microprobe. Therefore, the keystone did not need to be mounted on any support film that could cause contamination or limit the interpretation of the acquired data. The keystone was mounted within the microprobe target chamber with the largest dimension in the vertical axis. The top surface of the keystone was normal to the incident beam direction, and it was oriented so that the embedded particles were facing downstream from the incident beam.

\section{Scanning Transmission Ion Microscopy (STIM)}

For all analyses, an incident $3 \mathrm{MeV}$ proton beam was focused onto the keystone. As STIM uses $\sim 10^{6}$ lower beam currents than PESA, PBS, and PIXE, the initial analysis was by STIM. Using STIM, it is possible to quantify the column density of the silica aerogel and the embedded particles by measuring the energy lost from the $0.5 \mu \mathrm{m}$ focused incident beam as it traverses the sample (Lefevre et al. 1987). The simple physics involved in this ion energy loss mechanism, combined with available accurate databases, enable the ion energy losses to be readily converted into specimen-projected densities, which can be used to determine specimen mass (Lefevre et al. 1987; Lefevre et al. 1991; Bench et al. 1993). STIM has been used previously to determine the densities of thin material films of known density and composition (Bench 1991). Conversion of energy losses to densities using the known material composition established that the technique is quantitative, with ann accuracy $>95 \%$ in determining sample densities, as demonstrated in Bench (1991). The keystone sample was subjected to an initial coarse STIM scan that was 


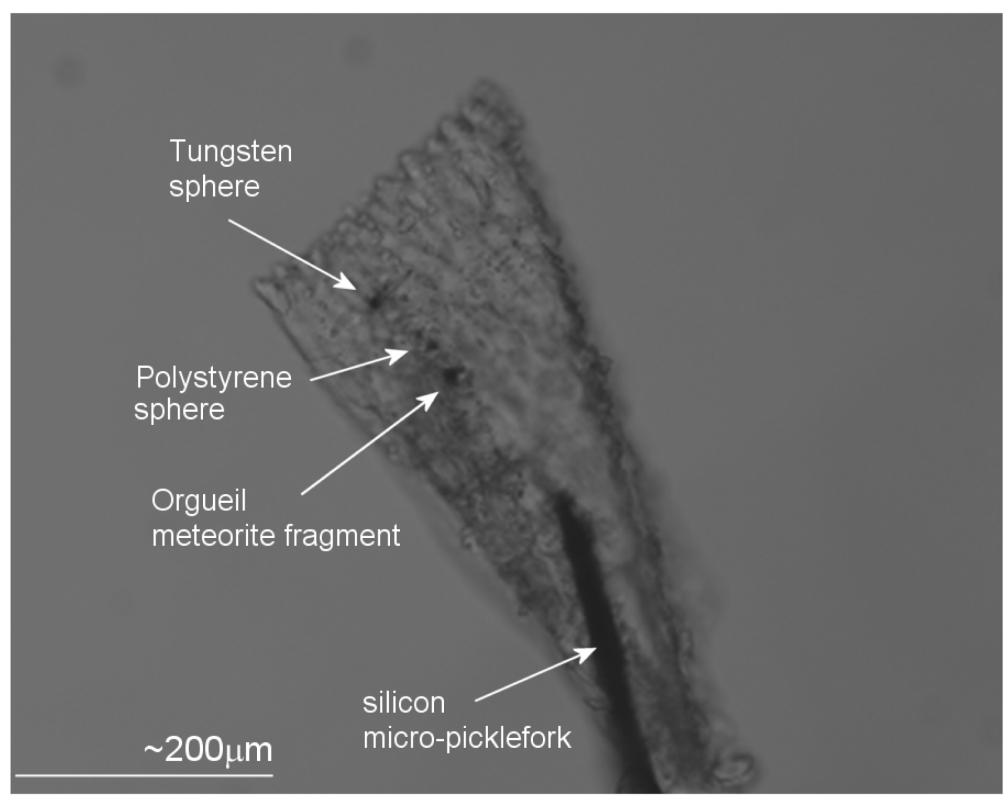

Fig. 2. Optical transmitted light micrograph of the silica aerogel keystone containing the embedded particles of tungsten, polystyrene, and Orgueil meteorite fragment. The keystone was extracted from bulk silica aerogel using the technique described in Westphal et al. (2004).

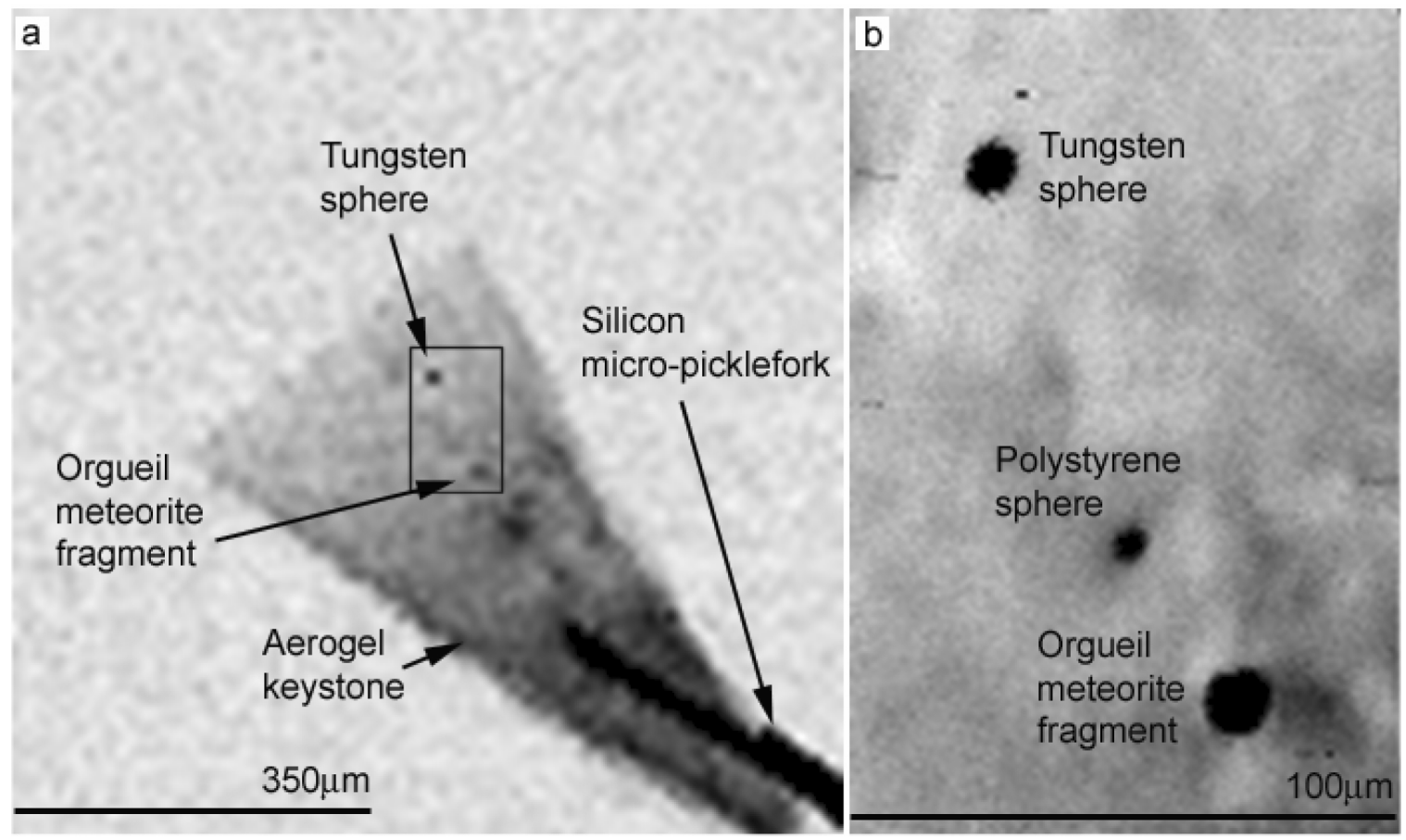

Fig. 3. a) STIM scan over the entire sample. In the image, it is clearly possible to see one of the supporting micro-forks. From the image, it is possible to see the variable column density of the silica aerogel; b) the high resolution scan of the region of interest highlighted in the coarse scan (black rectangular box) shows the particles of interest.

carried out over the entire sample (Fig. 3a) and then a high resolution scan over the area of interest containing the particles (Fig. 3b). From the STIM scans, it is noted that the silica aerogel has a variable density with an average density of $550 \pm 40 \mu \mathrm{g} / \mathrm{cm}^{2}$ (calculated from the high resolution scan). Despite the variation, the three embedded particles are clearly visible in the high resolution scan (Fig. 3b). Areal density $\left(\mu \mathrm{g} / \mathrm{cm}^{2}\right)$ is converted to mass by integrating the areal density of the particle over the area of the region of interest exposed to the particle beam. The tungsten sphere has a measured mass of $9.0 \pm 0.7 \mathrm{ng}$ that compares well to the predicated mass of $10.1 \mathrm{ng}$. The measured mass for the polystyrene 
sphere of $0.19 \pm 0.02 \mathrm{ng}$ agrees with its predicated mass of $0.19 \mathrm{ng}$. The Orgueil fragment has a measured mass of $2.6 \pm$ $0.4 \mathrm{ng}$. Error estimates are made by the addition in quadrature of $1 \sigma$ for counting statistics and error propagation of the following analysis parameters: pixel size, beam energy, energy loss equation, etc. The major advantage of STIM is that virtually every incident ion produces useful information in traversing the sample, i.e., the technique is nearly $100 \%$ efficient (Lefevre et al. 1991). Consequently, even with low beam currents, data acquisition is rapid. Therefore, a number of high resolution STIM scans could be used to locate fragmented debris; however, the experimental parameters used here do not allow for a complete assessment, and previous work has indicated that most material mass is lost during melting, a process that will occur during hypervelocity capture (Anderson and Ahrens 1994). It is also expected that Stardust will capture a wide range of materials from single crystal grains (e.g., forsterite) to complex heterogeneous particles similar to anhydrous IDPs. Even though the densities of these materials vary, it will be possible, with the current capabilities, to use STIM to rapidly select materials of immediate interest with a diameter of down to $0.5 \mu \mathrm{m}$. A complication to the application of STIM to "real" impact events is that the particles are typically coated with a condensed aerogel ablation shield that forms during the hypervelocity impact event (Hörz et al. 2000). This condensed material could lead to false STIM estimation of density; however, as we combine the STIM measurements with PIXE elemental analysis, it should be possible to discriminate between the ablation material and the particulate debris. Furthermore, any measurements acquired while the particles are still encapsulated must be viewed as only preliminary guidance to the composition and nature of the captured material.

By acquiring multiple STIM scans in multiple directions through a sample, it is also possible to carry out ion microtomography (IMT) (Pontau et al. 1989). Cross-sectional views and three-dimensional reconstructions can be acquired, both of which would be very useful in studying impact morphologies, as these are three-dimensional events.

\section{Proton Elastic Scattering Analysis (PESA) and Proton Backscattering (PBS)}

With PESA, protons with energies above $1 \mathrm{MeV}$ that elastically scatter from target atoms into forward angles can be detected by a particle detector (Cahill et al. 1987). Some of the kinetic energy of the projectile ion is absorbed by the recoiling target atom because the recoil energy depends on the target mass. By measuring the energy of the scattered ions, it is possible to discriminate between ions scattered from $\mathrm{H}$ and ions scattered by other elements. Integrating the number of ions scattered from $\mathrm{H}$ gives a quantitative measure of the sample hydrogen content. This technique works best for samples with small column densities in which proton-slowing by ordinary electronic stopping is small.

To acquire an $\mathrm{H}$ distribution map for the embedded particles (Fig. 4), the $3 \mathrm{MeV}$ proton beam was focused to a $2 \mu \mathrm{m}$ spot size as it was scanned across the region of interest within the keystone. A beam current of approximately $0.9 \mathrm{nA}$ and a charge of $1.5 \mu \mathrm{C}$ were deposited over the scanned region. The PESA data were recorded in list mode along with coincident beam spatial coordinates arising from scanning. The data was subsequently processed off-line using the inhouse ion microanalysis package (IMAP) developed by Lawrence Livermore National Laboratory/Sandia National Laboratories (Morse et al. 1999). To measure the $\mathrm{H}$ content of the polystyrene sphere and the Orgueil meteorite fragment, it was first necessary to calculate the $\mathrm{H}$ content of the silica aerogel background. The PESA data suggests that the silica aerogel has a variable $\mathrm{H}$ content that was averaged for the scanned region to be $0.29 \pm 0.02 \mu \mathrm{g} / \mathrm{cm}^{2}$. The $\mathrm{H}$ content for the polystyrene sphere was measured to be $10.2 \pm 1.5 \mathrm{pg}$ (the predicated mass is $14.6 \mathrm{pg}$ ), and the Orgueil fragment was $2.4 \pm 0.9 \mathrm{pg}$.

Coupled with the ability to detect $\mathrm{H}$ enrichments in situ, further identification of light elements $(\mathrm{C}, \mathrm{N}$, and $\mathrm{O})$ is possible by measuring the energies of the backscattered particles from the incident $3 \mathrm{MeV}$ proton beam that have collided elastically with the sample (Cookson 1987; Fraser 1995). The acquired proton backscattered spectrum will retain high-energy edges corresponding to the backscattering from atoms with different masses within the sample, thus making elemental detection possible (Fraser 1995). For proton backscattering analysis of the keystone, the detection is accomplished with a $100 \mathrm{~mm}^{2}$ silicon surface barrier particle detector. The detector is located $55 \mathrm{~mm}$ from the sample and subtended a mean scattering angle of 1600 . The particles were recorded in list mode along with coincident beam spatial coordinates arising from electrostatically scanning the $2 \mu \mathrm{m}$ in diameter beam spot. Data were reduced off-line, and spectra from features of interest within scanned regions were extracted and analyzed using IMAP (Morse et al. 1999) (Fig. 5). The spectrum acquired for the silica aerogel (Fig. 5a) detected, not surprisingly, $\mathrm{Si}$ and $\mathrm{O}$, but it also detected trace levels of $\mathrm{C}\left(2 \pm 1 \mu \mathrm{g} / \mathrm{cm}^{2}\right)$. It is not possible to state the nature of the $\mathrm{C}$ in terms of inorganic or organic components. However, identification of both $\mathrm{H}$ and $\mathrm{C}$ within the silica aerogel could indicate indigenous organic relics from the silica aerogel-forming process or background terrestrial contamination. Previous analyses of silica aerogels have indicated both of these to be the case (Hartmetz et al. 1990, Barrett et al. 1992, Wright et al. 1994). This indigenous organic material may be a problem for Stardust analysis because if, as expected, the cometary particles are similar to chondritic anhydrous IDPs, they are likely to be carbon-rich. Therefore, it could be impossible to unambiguously state the origin of organic material identified by in situ measurements. 


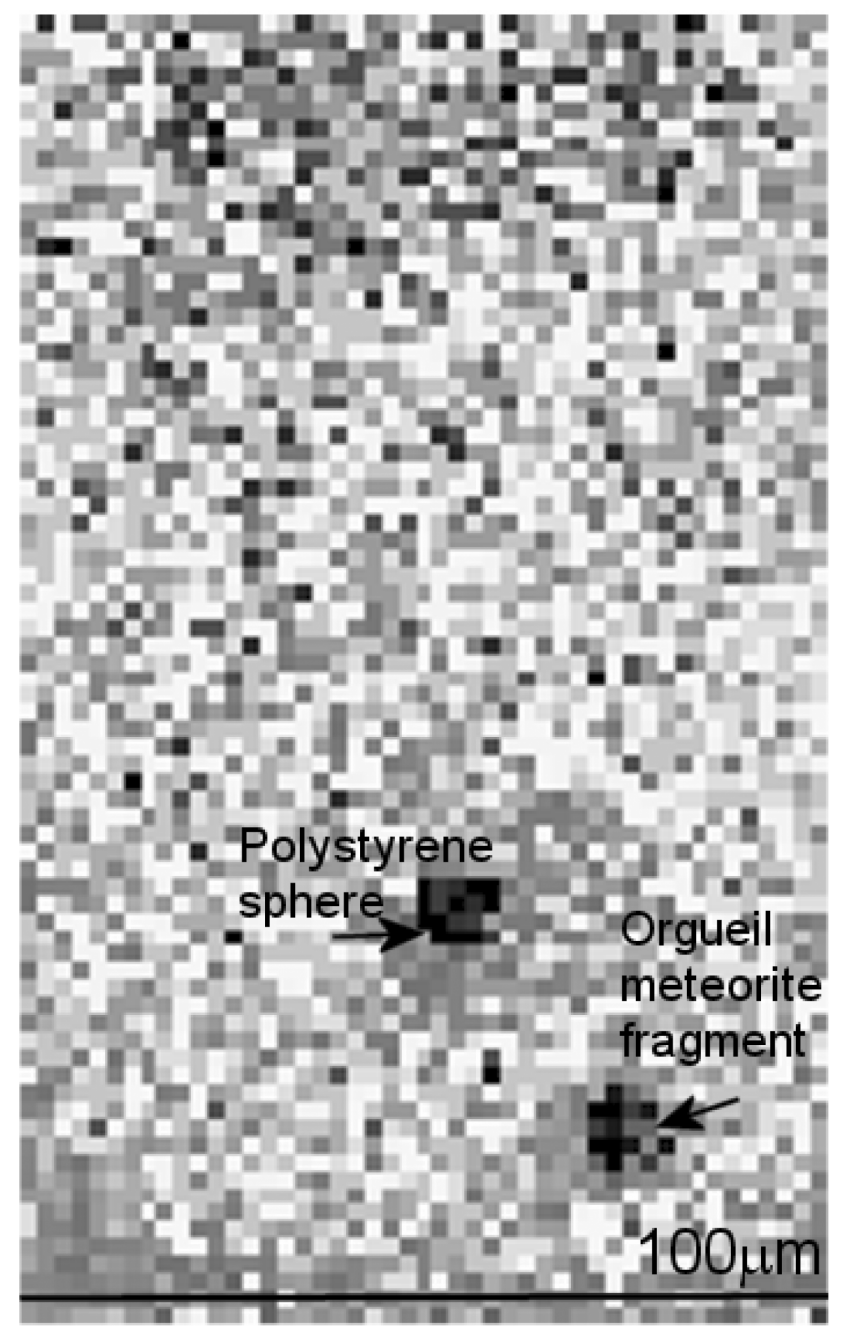

Fig. 4. Distribution of $\mathrm{H}$ acquired using PESA for the region of interest within the "keystone" that contains the embedded particles.

Detailed characterization of the Stardust flight-grade silica aerogel similar to the work carried out by Hartmetz et al. (1990) and Wright et al. (1994) before the return should enable a great degree of confidence in potential levels of organic contamination.

The PBS spectrum for the polystyrene (Fig. 5b) detected $\mathrm{C}$ and $\mathrm{O}$ with $\mathrm{Si}$ from the silica aerogel. The $\mathrm{C}$ content was measured as $150 \pm 20 \mathrm{pg}$, a level that compared favorably with the predicated $\mathrm{C}$ content of $174 \mathrm{pg}$. The PBS spectrum for the Orgueil fragment (Fig. 5c) was considerably different to the silica aerogel and the polystyrene sphere. No significant $\mathrm{C}$ was detected, and both the $\mathrm{Si}$ and $\mathrm{O}$ signals were background-corrected for the silica aerogel contributions. The analysis also detected $\mathrm{Fe}$ that could be associated with silicate or metallic phases within the fragment. The high Fe content of the particle and the overall size and geometry of the fragment in relation to the detector prevented the identification of the $\mathrm{C}$ (minimum detectable limit of approximately 200-300 pg) and
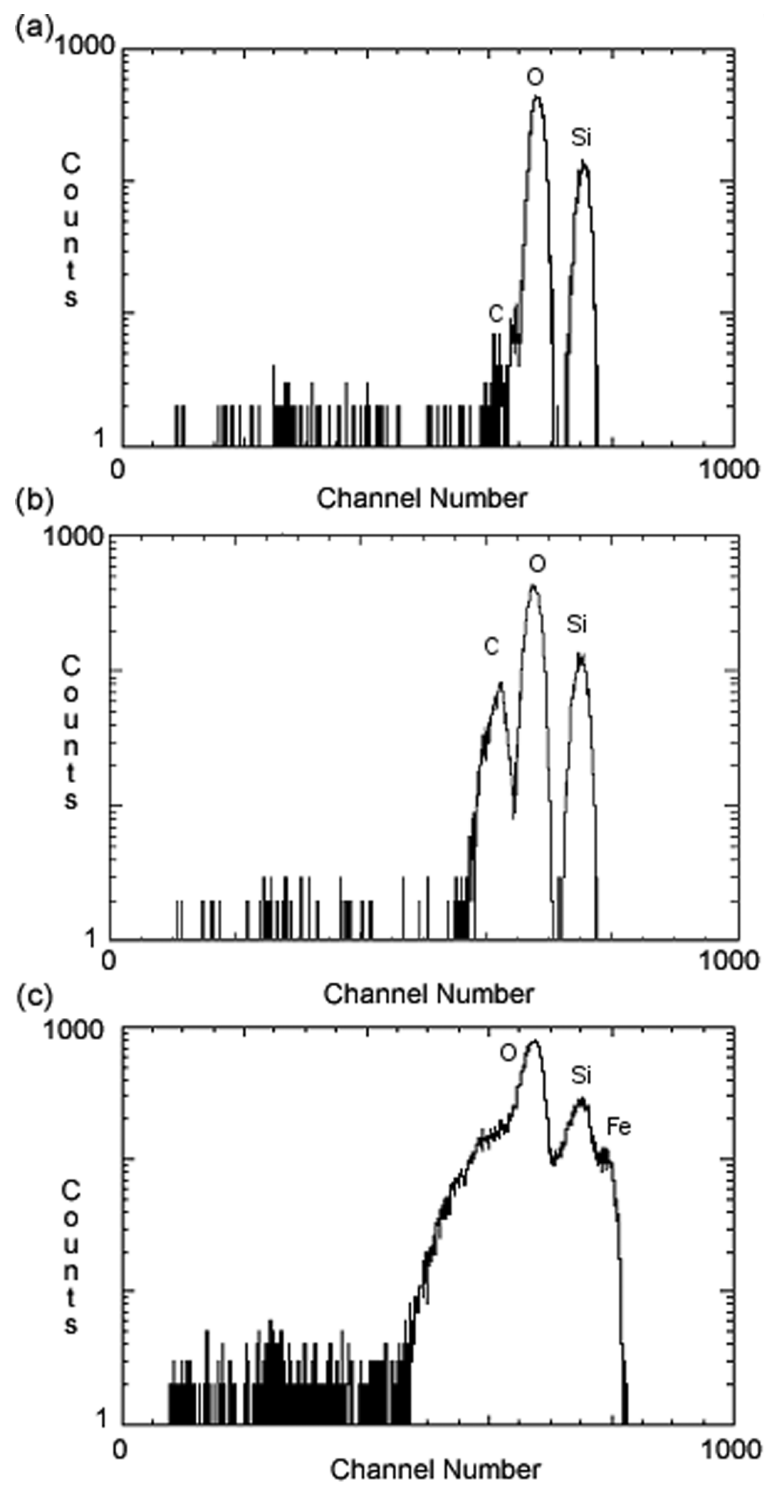

Fig. 5. a) Proton backscattered spectra acquired for the silica aerogel substrate; b) polystyrene sphere; c) Orgueil fragment. The spectra were obtained for a total beam charge of $1 \mu \mathrm{C}$.

the accurate quantification of the $\mathrm{O}$ content (approximately $800 \pm 400 \mathrm{pg})$.

While advances in instrumentation have lead to a new level of understanding the nature and origin of organic material in meteorites (e.g., Sephton 2002; Cody et al. 2002), the characterization of organic material in IDPs has been a much harder task due to the small sample size/concentration levels and it requires highly specialized analysis, e.g., tagging a sample using specific fluorescent molecular probe or CXANES analysis (Clemett et al. 2002; Flynn et al. 2003). Such analysis of the Stardust particles would certainly require that they are free from the silica aerogel. Notwithstanding the potential contamination problem, the combined analysis of PESA and PBS would enable a preliminary identification and 

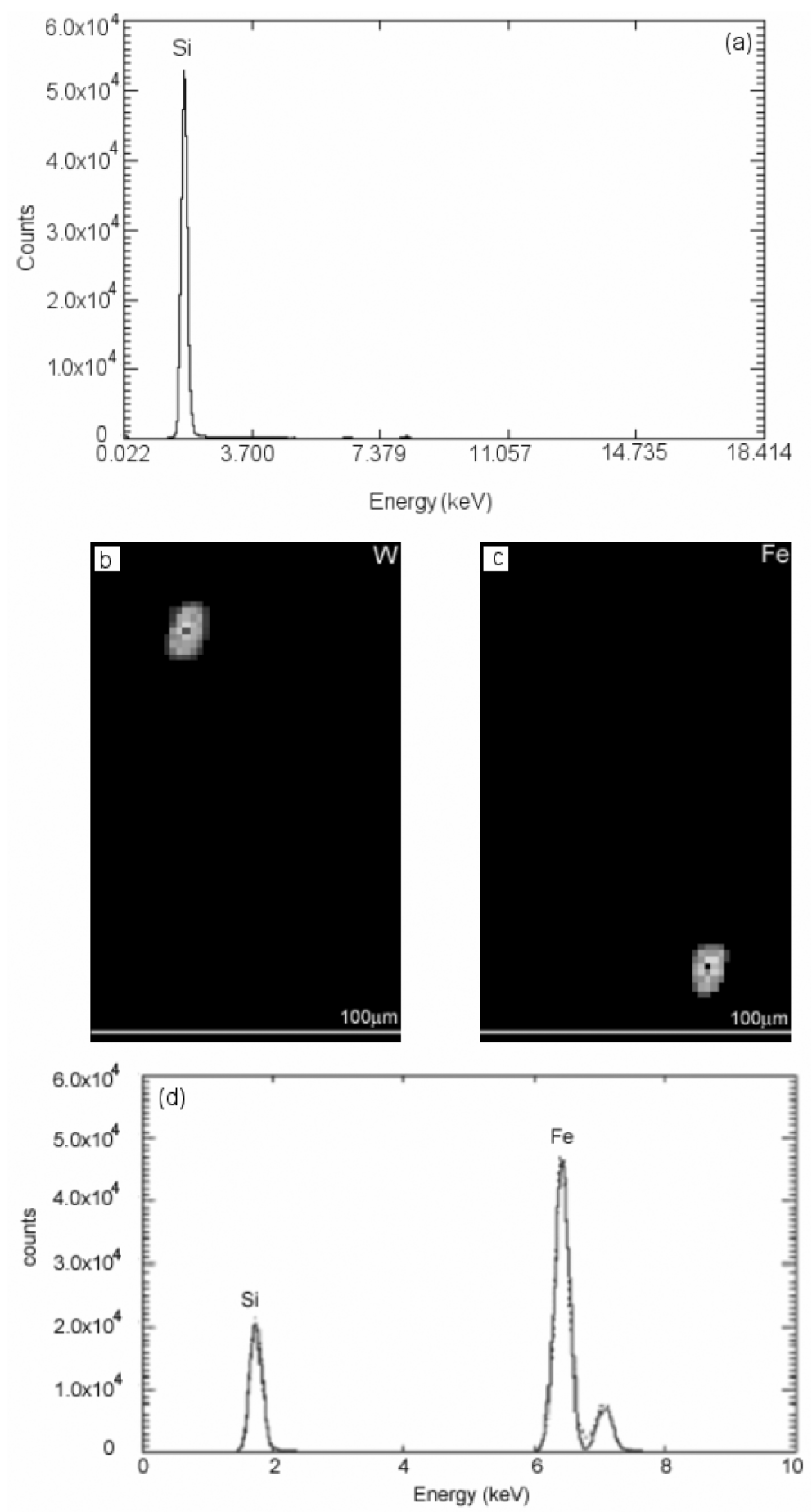

Fig. 6. a) PIXE spectrum acquired from the background aerogel; b) PIXE elemental map for W; c) PIXE elemental map acquired for distribution of $\mathrm{Fe}$ in the Orgueil meteorite fragment; d) PIXE X-ray spectrum acquired for the Orgueil fragment after processing.

screening for the C-rich particulates preserved in the silica aerogel, so these particulates could then be extracted and analyzed in further detail.

\section{Proton-Induced X-Ray Emission (PIXE)}

Of all the analytical techniques associated with nuclear microprobe analysis, PIXE is the most common and is used in material, geological, and planetary sciences (e.g., Fraser 1990; Arndt et al. 1996; Wies et al. 2002). PIXE uses a high-energy $(\mathrm{MeV})$ proton beam that interacts primarily with atomic electrons within the sample to create vacancies in inner shell orbitals. When an outer shell electron fills a vacancy, the excess energy resulting from the transition can be released as 
an X-ray photon, the energy of which is characteristic of the emitting atom. The proton-induced X-rays produced from the interaction between the $2 \mu \mathrm{m}$ focused $3 \mathrm{MeV}$ proton beam and the keystone were detected with an energy dispersive Iglet-X $\mathrm{X}$-ray detector, manufactured by Ortec, that subtended a solid angle of $\sim 200$ milli-steradians to the specimen. The detector is located at an angle of $135^{\circ}$ with respect to the incident beam. As the beam is scanned across the region of interest within the keystone, X-ray energy spectra are stored for each beam location. The total charge deposited over the scanned area was approximately $1 \mu \mathrm{C}$. After data acquisition, maps of element concentrations and X-ray spectra from beam locations corresponding to embedded particles were generated using IMAP (Morse et al. 1999) (Fig. 6).

The data acquired from the silica aerogel did not identify any elements with a $\mathrm{Z}$ of 12 or below (Fig. 6a). These may well be present, but the current configuration of the energydispersive X-ray detector inhibits the detection of low energy $\mathrm{X}$-rays due to the Be window used to protect it from moisture and backscattered protons. The analysis indicated that the density of $\mathrm{Si}$ was variable, with an average density for the scanned region of $284 \pm 30 \mu \mathrm{g} / \mathrm{cm}^{2}$. This value compares well with the calculated Si density measured by STIM of $260 \pm$ $20 \mu \mathrm{g} / \mathrm{cm}^{2}$ (assuming that the silica aerogel was composed solely of Si and O). PIXE is not particularly well-suited for light element detection, so no significant measurements were recorded for the polystyrene sphere. The main elemental data acquired from the PIXE analysis was for the tungsten sphere (Fig. 6b) and the Orgueil fragment (Figs. 6c-6d). From the PIXE data, the mass of the embedded tungsten sphere was measured to be $9.8 \pm 0.5 \mathrm{ng}$ and was in agreement with the measured STIM mass $(9.0 \pm 0.7 \mathrm{ng})$ and the calculated mass (10.1 ng). As the Orgueil fragment was extracted from the matrix of the meteorite, which is heterogeneous on the $\mu \mathrm{m}$ scale, it represented a more realistic analogue to the material that might be encountered by Stardust. The data acquired from the Orgueil fragment was background-corrected for the contribution of Si from the silica aerogel; the major elemental component identified was Fe (Figs. 6c-6d). The other detected elements are given in Table 1. At present, it is not possible to detect $\mathrm{Mg}$ at low levels, so we could not determine if the Fe was associated with a silicate or simply a metallic phase (oxide or metal). Later characterization by X-ray diffraction at the Natural History Museum in London found the major mineralogical composition to be magnetite $\left(\mathrm{Fe}_{3} \mathrm{O}_{4}\right)$.

Using PIXE, we have successfully imaged and quantitatively analyzed elements with $Z>11$ in the embedded particles. However, it has been highlighted in this work and by previous researchers (Zolensky et al. 2000) that there are significant detection limits with light elements that are beyond the quantitative capabilities of the current Ortec Iglet$\mathrm{X} X$-ray detector used. The analytical range of the microprobe for the sensitive quantitative analysis could be extended to include elements from $\mathrm{C}$ to $\mathrm{Na}$ and to increase the sensitivity
Table 1. Elemental data acquired from the embedded Orgueil meteorite fragment with a mass of $2.6 \pm 0.4 \mathrm{ng}$ (from the acquired STIM data) using analysis techniques associated with the nuclear microprobe at LLNL.

\begin{tabular}{llcc}
\hline \multirow{2}{*}{ Technique } & Element & Content $(\mathrm{pg})$ & $\begin{array}{c}\text { Wt\% of } \\
\text { STIM mass }\end{array}$ \\
\hline PESA & $\mathrm{H}$ & $2.4 \pm 0.9$ & 0.1 \\
PIXE & $\mathrm{Al}$ & $10 \pm 3$ & 0.4 \\
PIXE & $\mathrm{Si}$ & $83 \pm 24$ & 3.2 \\
PIXE & $\mathrm{S}$ & $36 \pm 6$ & 1.4 \\
PIXE & $\mathrm{Ca}$ & $5 \pm 2$ & 0.2 \\
PIXE & $\mathrm{Cr}$ & $2.6 \pm 0.3$ & 0.1 \\
PIXE & $\mathrm{Mn}$ & $2.4 \pm 0.4$ & 0.1 \\
PIXE & $\mathrm{Fe}$ & $1330 \pm 140$ & 51.9 \\
PIXE & $\mathrm{Ni}$ & $16 \pm 2$ & 0.6 \\
PBS & $\mathrm{O}$ & $800 \pm 400$ & 31 \\
\multicolumn{2}{l}{ Sum of STIM mass $\mathrm{wt} \%$} & & 89.0 \\
\hline
\end{tabular}

for the detection of $\mathrm{Mg}$ and $\mathrm{Al}$ by using a windowless detector.

\section{Disadvantages of Nuclear Microprobe Analysis}

There are several limitations in using the nuclear microprobe for in situ analysis. First, it has already been shown that, with the current $\mathrm{Be}$ window and energy dispersive detectors, there is a limit in the detection of light elements. Second, while STIM is essentially non-destructive because the technique uses million-fold lower beam currents than the other techniques (PESA, PBS, and PIXE), which are generally assumed to be only partially non-destructive, as there is potential for sample damage from the higher beam currents employed. Previous researchers have studied the alteration of stoichiometry in a sample due to the loss of $\mathrm{H}$ and $\mathrm{O}$ during repeated PESA analysis (Delto et al. 2002). However, it is possible to limit the potential beam damage by reducing the overall total charge deposited on the sample during the analysis. Furthermore, collecting PESA, PBS, and PIXE concurrently can reduce the acquisition time for data collection.

\section{FOCUSED ION BEAM MICROMACHINING OF SILICA AEROGEL}

When developing a strategy for the characterization of the cometary particles to be returned by Stardust, it is important to have analytical techniques that can be employed in the preliminary stages for the post-flight program to study aerogel encapsulated cometary material. However, detailed mineralogical and chemical investigations will need sample preparation and almost certainly will require the particulate fragments to be free of silica aerogel. Westphal et al. (2002) showed that individual particles as small as $3 \mu \mathrm{m}$ in diameter could be extracted intact from silica aerogel. However, it is 


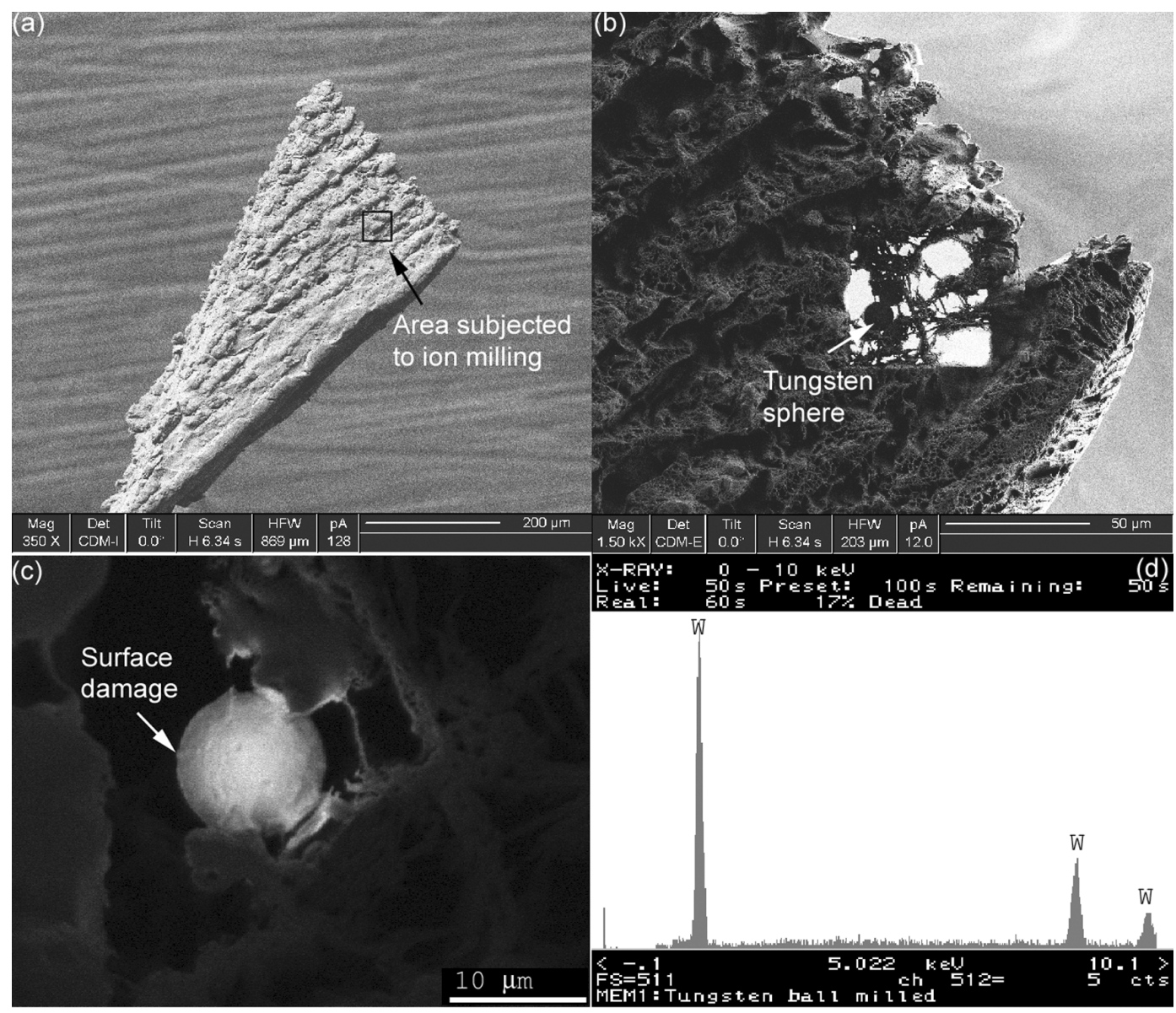

Fig. 7. a) Ion micrograph of the intact silica aerogel keystone; b) secondary electron micrograph of the keystone after the encapsulating silica aerogel has been removed by rastering the $\mathrm{Ga}^{+}$ion beam across the selected $50 \mu \mathrm{m} \times 45 \mu \mathrm{m}$ rectangular area. The tungsten sphere is clearly visible in the center of the image; c) backscattered electron micrograph of the tungsten sphere. The white arrow locates the slight surface damage sustained by the tungsten sphere by the $\mathrm{Ga}^{+}$ions; $\mathrm{d}$ ) the X-ray energy dispersive spectrum acquired for the tungsten sphere.

unlikely that the same technique could be employed on sub$\mu \mathrm{m}$-sized fragments.

Ion beam techniques can be used for analysis and can also be employed to micromachine or microfabricate materials. Focused ion beam (FIB) microscopy was principally used in the semiconductor industry, as it enabled the in situ manipulation and machining of materials on the sub-micron scale (e.g., De Veirman and Weaver 1999; Lábár and Egerton 1999). FIB microscopy uses a focused beam of $\mathrm{Ga}$ ions to ablate volumes of material from precise locations and at carefully controlled rates (see Phaneuf [1999] for a detailed discussion on design and operation of the FIB microscope). The significant benefit of the technique is that the sample can be imaged with $\mathrm{nm}$-scale resolution during the ion milling using both ion and secondary electron imaging. The technique has been used in both geological and planetary sciences to prepare electron-transparent thin sections of materials (Heaney et al. 2001; Stroud et al. 2002; Stroud 2003; Lee et al. 2003). Apart from this more traditional use of FIB, it has also been shown that the technique can be used to remove the silica aerogel film that coats the surface of particles that have been laboratory impacted and subsequently recovered from silica aerogel (e.g., Graham et al. 2002). This suggested that FIB could be used to expose particulates in the silica aerogel.

Using an FEI 200TEM workstation, the tungsten sphere and the Orgueil fragment have been subjected to sitespecific ion milling to remove the silica aerogel covering them. From the initial ion image of the keystone, it was not possible to locate the particles. However, it was possible to match topographic features from the optical transmitted light micrograph of the keystone (Fig. 2) or the STIM image (Fig. 3) and the ion image (Fig. 7a) to indicate the approximate location of the tungsten sphere. An $50 \mu \mathrm{m} \times$ $45 \mu \mathrm{m}$ area was selected around this region of interest, and the silica aerogel was subsequently removed by rastering the $400 \mathrm{~nm}$ spot-sized gallium ion beam at $30 \mathrm{kV}$ with a $20 \mathrm{nA}$ beam current until the tungsten sphere was exposed (Fig. 7b). During the milling that took approximately $7 \mathrm{~min}$, the entire process was monitored by collecting secondary electron images. Despite the monitoring, the silica aerogel was ablated at such a rapid rate that the top surface of the 
tungsten sphere was also subjected to slight ion milling (Fig. 7c). It is also clear from the acquired images that the ion beam can have detrimental effects on the supporting silica aerogel. This is evident because there is a distinct difference in the edge of the silica aerogel between the initial ion image and the secondary electron image acquired after the milling, as some material has been lost. Additional imaging and microanalysis of the tungsten sphere were carried out using a conventional JEOL 840 scanning electron microscope (Figs. 7c-7d). It would be expected that, after in situ characterization, exposed particles would be removed from the supporting silica aerogel using a micromanipulator.

If the observations from particles captured on LEO exposed silica aerogel collectors provide an accurate analogue to how the Stardust particles will be preserved, then it is entirely possible that some fragments may be on a scale that prohibits intact extraction. Therefore, this material would have to be analyzed in situ. As has already been discussed, there are limitations to such analysis particularly because of backgrounds due to contamination in the silica aerogel. However, FIB can be used to remove the encapsulating silica aerogel and also to prepare a polished section of sample while it is still surrounded by silica aerogel. The Orgueil fragment was subjected to an initial milling procedure (i.e., ion beam focused to $400 \mathrm{~nm}$ with a $20 \mathrm{nA}$ beam current) similar to that of the tungsten sphere to remove the silica aerogel. The entire sample was then tilted to $45^{\circ}$, and the Orgueil grain was subjected to further ion milling at initially high beam currents to remove the outer surface of the grain but then at progressively lower currents of 10000, 7000, and then $5000 \mathrm{pA}$ to clean up the milled surface (Fig. 8a). After the sample is orientated back to a normal $0^{\circ}$ of tilt, the top surface of the grain has now been polished flat (Fig. 8b), and the sample can be analyzed using a number of electron and ion beam techniques (Fig 8c). By preparing the sample this way, any contaminating silica aerogel is removed.

There are a number of disadvantages to using FIB for particle extraction. First, compared to an optical system, the instrumentation is very expensive (a typically system costs around \$1 million). Second, during the ion milling process, it is possible to generate imaging artifacts at the high beam currents, although these are typically removed by the final "cleaning" stages of the milling procedure that are carried out at lower beam currents (Fig. 8b). Third, it is possible to deposit an amorphous film (nanometer thick) on the surface that has been milled during the higher beam current stages. Again, this can be nearly completely removed by the final stages of the sample preparation (Lee et al. 2003). Perhaps one of the most significant disadvantages is that older FIB microscopes use the ion beam to mill and image the sample. It would appear that prolonged exposure of the silica aerogel under the ion beam could cause it to disintegrate. This potential problem can also be resolved by the fact that modern
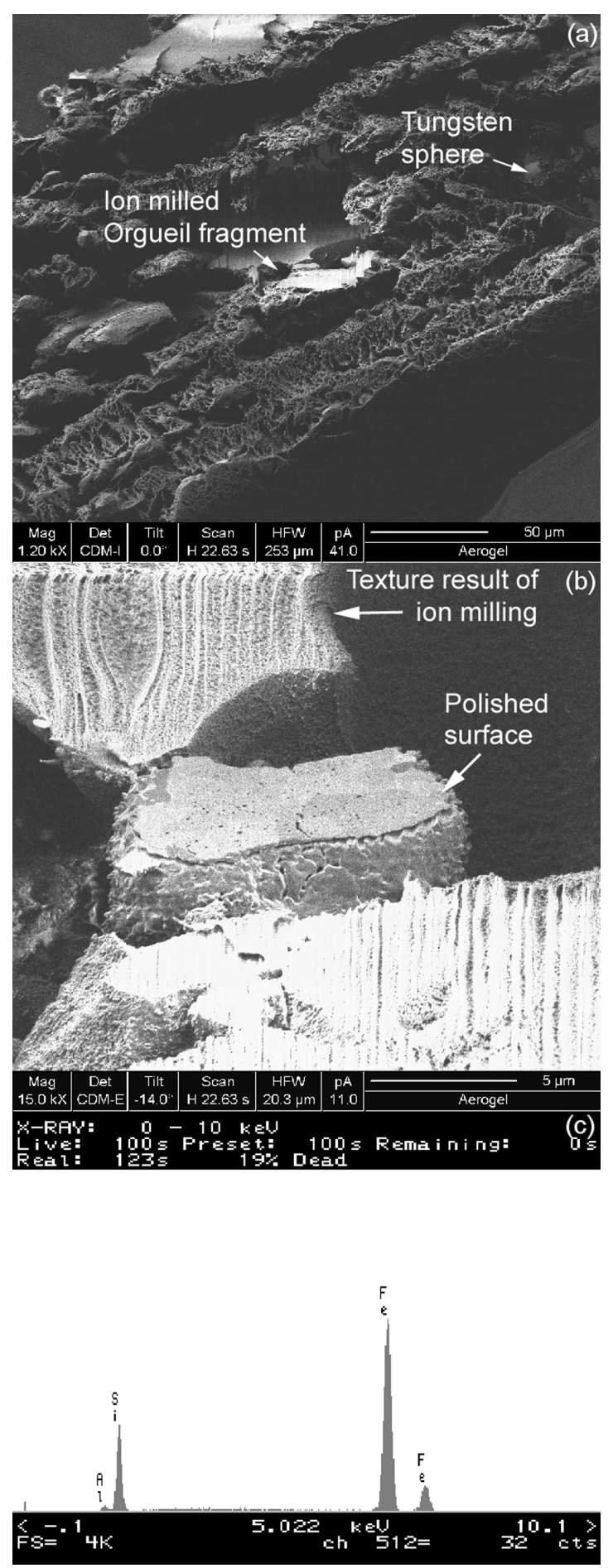

Fig. 8. a) Secondary electron micrograph of the embedded Orgueil meteorite after the top surface of aerogel has been removed by the ion milling; b) secondary electron micrograph of the fragment after the ion beam has been used to produce a polished flat surface. The polished surface appears to show some structural or crystallographic phase information. The surface structure observed on the surrounding silica aerogel (indicated by a white arrow) is an artifact of the milling process; c) the X-ray energy dispersive spectrum acquired for the fragment. 
fully equipped FIB systems use dual beam technologies: all the imaging is carried out using the electron beam, while the ion beam is used for the milling. Furthermore, these newer instruments now typically include a scanning transmission electron microscopy detector (for high resolution imaging), electron backscattered diffraction detection (for crystallographic analysis), and energy-dispersive X-ray detection (for elemental analysis).

Since there are multiple potential benefits from using an "all-in-one" instrument to prepare and chemically analyze samples at the initial stages of particle characterization, it is clear that FIB microscopy will play a significant role in the characterization of Stardust materials.

\section{SUMMARY}

The observation that micrometeoroids captured in LEO fragment upon impact suggests that the cometary particles captured by Stardust will also have fragmented. Therefore, it is important to develop techniques capable of analyzing embedded materials. The nuclear microprobe at Lawrence Livermore National Laboratory has been successfully used to characterize embedded particles in low-density silica aerogel using STIM, PESA, PBS, and PIXE. The combination of these techniques allows for the detection of both inorganic and organic components within a particle, while still encapsulated in silica aerogel.

While it is important to have the capability of acquiring measurements from embedded particles, further investigations of mineralogy and chemistry will require the particles to be extracted from the silica aerogel. This was originally shown to be possible using a mechanical recovery technique (Westphal et al. 2002, 2004). It has now been shown that FIB microscopy can be used to rapidly ablate the silica aerogel encapsulating embedded particles, leaving the particles exposed and in a configuration suitable for further detailed characterization while still in the silica aerogel or after extraction using micromanipulators. We will now employ these techniques on micrometeoroids captured in collectors that have previously been exposed in LEO.

By applying and learning from these techniques now, we will be ready for the return of the precious Stardust particles in 2006 .

Acknowledgments-This work was performed under the auspices of the U.S. Department of Energy, National Nuclear Security Administration by the University of California and Lawrence Livermore National Laboratory under contract No. W-7405-Eng-48. The work carried out by Andrew Westphal, Chris Snead, and Anna Butterworth at the University of California at Berkeley is supported by NASA grant NAG511902. Chris Keller (MEMSPI) is thanked for manufacturing the silicon microforklifts. We also thank Dan Morse, Randy Leber, Skip Fields, Sean Watson, Daniel Espinosa, Joe Ruth, and Tom Brown for support and assistance with nuclear microprobe operation. Gordon Cressey (The Natural History Museum, London) and Phil Bland (Imperial College, London) are also thanked for the XRD characterization of the Orgueil meteorite fragment. Hope Ishii is thanked for constructive comments on the manuscript. We also wish to thank F. Hörz and L. P. Keller, whose reviews contributed greatly to this manuscript.

\section{Editorial Handling-Dr. Don Brownlee}

\section{REFERENCES}

Anderson W. W. and Ahrens T. J. 1994. Physics of interplanetary dust capture via impact into organic polymer foams. Journal of Geophysical Research 99:2063-2071.

Arndt P., Bohsung J., Maetz M., and Jessberger E. K. 1996. The elemental abundances in interplanetary dust particles. Meteoritics 28:490-514.

Barrett R. A., Zolensky M. E., Hörz F., Lindstrom D., and Gibson E. K. 1992. Suitability of silica aerogel as a capture medium for interplanetary dust. Proceedings, 22nd Lunar and Planetary Science Conference. pp. 203-212.

Bench G. S. 1991. Scanning transmission ion microscopy. Ph.D thesis, University of Melbourne, Melbourne, Australia.

Bench G. S., Saint A., Legge G. J. F., and Cholewa M. 1993. Applications of energy loss contrast STIM. Nuclear Instruments and Methods in Physics Research B 77:175-183.

Bernhard R. P., Warren J., See T. H., Borg J., Quirico E., Simionovici A., Raynal P. I., Chevallier P., and Langevin Y. 2002. Synchrotron radiation as a tool for in situ investigation of extraterrestrial grains in low-density collectors: Application to the analyses of the PIE polymid foam targets. Planetary and Space Science 50:1055-1065.

Brownlee D. E., Hörz F., Hrubesh L., McDonnell J. A. M., Tsou P., and Williams J. 1994. Eureka! Aerogel capture of micrometeoroids in space (abstract). 25th Lunar and Planetary Science Conference. pp. 183-184.

Brownlee D., Tsou P., Burnett D., Clark B., Hanner M. S., Hörz F., Kissel, J., McDonnell J. A. M., Newburn R. L., Sandford S., Sekanina Z., Tuzzolino A. J., and Zolensky M. E. 1997. The Stardust mission: Returning comet samples to earth (abstract). Meteoritics \& Planetary Science 32: A22.

Brownlee D. E., Anderson J. D., Atkins K., Bhaskaran S., Cheuvront A. R., Clark B. C., Duxbury T. C., Economou T., Hanner M. S., Hörz F., Kissel J., McDonnell J. A. M., Green S., Newburn R. L., Perkins D. E., Price S., Ryan R. E., Sandford S., Sekanina Z., Tsou P., Tuzzolino A. J., Villinga J. M., Williams K. E., and Zolensky M. E. 2004. The Stardust-A successful encounter with the remarkable comet Wild 2 (abstract \#1981). 35th Lunar and Planetary Science Conference. CD-ROM.

Burchell M. J., Creighton J. A., Cole M. J., Mann J., and Kearsley A. T. 2001. Capture of particles in hypervelocity impacts in aerogel. Meteoritics \& Planetary Science 36:209-221.

Cahill T. A., Eldred R. A., Wallace D., and Kusko B. H. 1987. The hygrogen-sulfur correlation, by PIXE plus PESA, and the aerosol source identification. Nuclear Instruments and Methods in Physics Research B 22:296-300.

Clemett S. J., Messenger S., Keller L. P., Thomas-Keprta K. L., and McKay D. S. 2002. Spatially resolved analysis of amines in interplanetary dust particles using molecular probes (abstract). Meteoritics \& Planetary Science 37:A36. 
Cody G. D., Alexander C. M. O'D., and Tera F. 2002. Solid-state $\left({ }^{1} \mathrm{H}\right.$ and ${ }^{13} \mathrm{C}$ ) nuclear magnetic resonance spectroscopy of insoluble organic residue in the Murchison meteorite: A self-consistent quantitative analysis. Geochimica et Cosmochemica Acta 66: $1851-1865$.

Cookson J. A. 1987. Microbeam applications in metallurgy and industry. In Principles and applications of high-energy ion microbeams, edited by Watt F. and Grime G. W. Bristol: Adam Hilger. pp. 273-298.

Dai Z. R., Bradley J. P., Joswiak D. J., Brownlee D. E., Hill H. G. M., and Genge M. M. 2002. The origins of meteoritic nanodiamonds. Nature 418:157-159.

Delto R., Samlenski R., and Brenn R. 2002. MeV microprobe analysis of polymer films: Radiation damage aspects. Nuclear Instruments and Methods in Physics Research B 190:318-323.

De Veirman A. and Weaver L. 1999. The use of a focused-ion-beam machine to prepare transmission electron microscopy samples of residual photoresist. Micron 30:213-220.

Flynn G. J. and Keller L. P. 2003. Analysis of the organic matter in interplanetary dust particles: Clues to the organic matter in comets, asteroids, and interstellar grains (abstract). In Workshop on Cometary Dust in Astrophysics. LPI Contribution No. 1182. Houston: Lunar and Planetary Institute. 29 p.

Flynn G. J., Sutton S. R., and Hörz F. 2001. Synchrotron X-ray microprobe in situ trace element analysis of "swarm" particles collected in aerogel on the Mir space station: Evidence for a CIlike composition (abstract \#1398). 32nd Lunar and Planetary Science Conference. CD-ROM.

Flynn G. J., Lanzirotti A., Westphal A. J., Domínguez G., and Snead C. 2003. Chemical and mineralogical analysis of an extraterrestrial particle in aerogel (abstract \#1814). 34th Lunar and Planetary Science Conference. CD-ROM.

Fraser D. G. 1990. Applications of the high resolution scanning proton microprobe in the earth sciences: An overview. Chemical Geology 83:27-37.

Fraser D. G. 1995. The nuclear micrprobe-PIXE, PIGE, RBS, NRA, and ERDA. In Microprobe techniques in earth sciences, edited by Potts P. J., Bowles J. F. W., Reed S. J. B., and Cave M. R. London: Chapman \& Hall. pp. 143-162.

Graham G. A., Franchi I. A., Kearsley A. T., and Burchell M. J. 2001. The use of a Raman microprobe in analysis of extraterrestrial material impacted into aerogel. Proceedings of the Royal Microscopical Society 36:251-254.

Graham G. A., Chater R. J., McPhail D. S., Kearsley A. T., Lee M. R., Kettle S., and Wright I. P. 2002. In situ sectioning and analysis of cosmic dust using focused ion beam microscopy. Meteoritics \& Planetary Science 37:A56.

Graham G. A., Kearsley A. T., Butterworth A. L., Bland P. A., Burchell M. J., McPhail D. S., Chater R., Grady M. M., and Wright I. P. Forthcoming. Extraction and microanalysis of cosmic dust captured during sample return missions. Advances in Space Research.

Hartmetz C. P., Gibson E. K., Jr., and Blanford G. E. 1990. In situ extraction and analysis of volatiles and simple molecules in interplanetary dust particles, contaminants, and silica aerogel. Proceedings, 20th Lunar and Planetary Science Conference. pp. 343-355.

Heaney P. J., Vicenzi E. P., Giannuzzi L. A., and Livi K. J. T. 2001. Focused ion beam milling: A method of site-specific sample extraction for microanalysis of Earth and planetary materials. American Mineralogist 86:1094-1099.

Hörz F., Cintala M. J., Zolensky M. E., Bernhard R. B., Davidson W. E., Haynes G., See T. H., Tsou P., and Brownlee D. E. 1998. Capture of hypervelocity particles with low-density aerogel. NASA TM-98-201792.
Hörz F., Zolensky M. E., Bernhard R. P., See T. H., and Warren J. L. 2000. Impact features and projectile residues in aerogel exposed on Mir. Icarus 147:559-579.

Kearsley A.T., Franchi I. A., Graham G. A., and Burchell M. J. 2001. Characterization of extraterrestrial samples by Raman and electron microprobes (abstract). Meteoritics \& Planetary Science 36:A94.

Keller L. P., Hony S., Bradley J. P., Molster F. J., Waters L. B. F. M., Bouwman J., de Koter A., Brownlee D. E., Flynn G. J, Henning T., and Mutschke H. 2002. Identification of iron sulphide grains in protoplanetary disks. Nature 417:148-150.

Keller L. P., Flynn G. J., and Westphal A. J. 2003. Interplanetary dust and particles captured in aerogel: Far-infrared spectroscopy (abstract). Meteoritics \& Planetary Science 38:A148.

Lábár J. L. and Egerton R. 1999. Special issue on ion beam techniques. Micron 30:195-196.

Lee M. R., Bland P. A., and Graham G. 2003. Preparation of TEM samples by focused ion beam (FIB) techniques: Applications to the study of clays and phyllosilicates in meteorites. Mineralogical Magazine 67:581-592.

Lefevre H. W., Schofield R. M. S., Overley J. C., and McDonald J. C. 1987. Scanning transmission ion microscopy as it complements particle induced X-ray emission microscopy. Scanning Microscopy 1:879-889.

Lefevre H. W., Schofield R. M. S., Bench G. S., and Legge G. J. F. 1991. STIM with energy loss contrast: An imaging modality unique to $\mathrm{MeV}$ ions. Nuclear Instruments and Methods in Physics Research B 54:363-370.

Messenger S., Keller L. P., Stadermann F. J., Walker R. M., and Zinner E. 2003. Samples of stars beyond the solar system: Silicate grains in interplanetary dust. Science 300:105-108.

Morse D. H., Antolak A. J., Bench G. S., Heikkinen D. W., Roberts M. L., and Sideras-Haddad E. 1997. Materials analysis at the SNL/LLNL nuclear microprobe. Nuclear Instruments and Methods in Physics Research B 130:740-745.

Morse D. H., Antolak A. J., Bench G. S., and Roberts M. L. 1999. A flexible LabVIEW ${ }^{\mathrm{TM}}$-based data acquisition and analysis system for scanning microscopy. Nuclear Instruments and Methods in Physics Research B 158:146-152.

Phaneuf M. W. 1999. Applications of focused ion beam microscopy to material science specimens. Micron 30:277-288.

Pontau A. E., Antolak A. J., Morse D. H., Ver berkmoes A. A., Brase J. M., Heikkinen D. W., Martz H. E., and Proctor I. D. 1989. Ion microbeam tomography. Nuclear Instruments and Methods in Physics Research B 40/41:646-650.

Raynal P. I., Quirico E., Borg J., Deboffle D., Dumas P., d'Hendecourt L., Bibring J. P., and Langevin Y. 2000. Synchrotron infrared microscopy of micron-sized extraterrestrial grains. Planetary and Space Science 48:1329-1339.

Sephton M. A. 2002. Organic compounds in carbonaceous meteorites. Natural Product Reports 19:292-311.

Stadermann F. J. and Floss C. 2000. SIMS isotopic analysis of interplanetary dust from space exposed aerogel (abstract \#1372). 30th Lunar and Planetary Science Conference. CD-ROM.

Stephan T. 2000. TOF-SIMS in cosmochemistry. Planetary and Space Science 49:859-906.

Stroud R. M., O'Grady M., Nittler L. R., and Alexander C. M. O'D. 2002. Transmission electron microscopy of an in situ presolar silicon carbide grain (abstract \#1785). 33rd Lunar and Planetary Science Conference. CD-ROM.

Stroud R. M. 2003. Focused ion beam microscopy of extraterrestrial materials: Advances and limitations (abstract). In Workshop on Cometary Dust in Astrophysics. LPI Contribution No. 1182. Houston: Lunar and Planetary Institute. 72 p.

Tsou P. 1995. Silica aerogel captures cosmic dust intact. Journal of 
Non-Crystalline Solids 186:415-427.

Westphal A. J., Snead C., Borg J., Quirico E., Raynal P. I., and Zolensky M. E. 2002. Small hypervelocity particles captured in aerogel collectors: Location, extraction, handling, and storage. Meteoritics \& Planetary Science 37:855-865.

Westphal A. J., Snead C., Butterworth A., Graham G. A., Bradley J. P., Bajt S., Grant P. G., Bench G., Brennan S., Pianetta P. 2004. Aerogel keystones: Extraction of complete hypervelocity impact events from aerogel collectors. Meteoritics \& Planetary Science 39:1375-1386.

Wies C., Jessberger E. K., Klöck W., Maetz M., Rost D., Stephan T.,
Traxel K., and Wallianos A. 2001. Mineral-specific trace element contents of interplanetary dust particles. Nuclear Instruments and Methods in Physics Research B 181:539-544.

Wright I. P., Huang H. P., and Pillinger C. T. 1994. Attempts to produce carbon-free silica aerogel for micrometeoroid capture cells (abstract). 25th Lunar and Planetary Science Conference. pp. $1515-1516$.

Zolensky M. E., Pieters C., Clark B., and Papike J. J. 2000. Small is beautiful: The analysis of nanogram-sized astromaterials. Meteoritics \& Planetary Science 35:9-29. 
Ishii H.A., Brennan S., Luening K., Pianetta P., Bradley J.P., Snead C.J. and Westphal A.J. (2005) Hard x-ray spectromicroscopy techniques at SSRL for astromaterials analysis. 36th Lunar and Planetary Science Conference (League City, TX, March 2005), CD-ROM: Abstract 1393. UCRL-ABS208887. 
HARD X-RAY SPECTRO-MICROSCOPY TECHNIQUES AT SSRL FOR ASTROMATERIALS ANALYSIS. H.A. Ishii ${ }^{1}$, S. Brennan ${ }^{2}$, K. Luening ${ }^{2}$, P. Pianetta ${ }^{2}$, J.P. Bradley ${ }^{1}$, C.J. Snead ${ }^{3}$ and A.J. Westphal ${ }^{3}$, Bay Area Particle Analysis Consortium, ${ }^{1}$ Institute for Geophysics and Planetary Physics, Lawrence Livermore National Laboratory, Livermore, CA 94550, USA (hope.ishii@1lnl.gov), ${ }^{2}$ Stanford Synchrotron Radiation Laboratory, Stanford Linear Accelerator Center, Stanford, CA 94025, USA (brennan@stanford.edu), ${ }^{3}$ Space Science Laboratory, University of California at Berkeley, Berkeley, CA 94720, USA.

Introduction: Sample return missions allow the laboratory study of material from other parts of our solar system which until recently were accessible only by astronomical observation. In January of 2006, NASA's Stardust Mission will return to Earth with particles captured in silica aerogel collected from the coma of Comet Wild-2 as well as fresh interstellar dust. The primary objective of the mission is the collection of 1000 analyzable particles of diameter $>15$ micron [1]. Estimates from on-board instrumentation indicate 3 times that number were collected [2]. Each of these femto- to nanogram particles is potentially a heavyweight in terms of scientific return.

Scientific and technical preparations for Stardust's return have long been underway via analysis of analogues like interplanetary dust particles (IDPs) collected in the stratosphere and micrometeoroids captured in low earth orbit in aerogel collectors (e.g. the Orbital Debris Collector Experiment). To study such small volumes of material non-destructively, synchrotron radiation techniques have been used with increasing frequency [ 3 and references therein, 4, 5]. These techniques include microdiffraction to determine mineralogy, X-Ray Fluorescence spectroscopy (XRF) to determine elemental compositions and distributions, Fourier Transform Infrared (FTIR) spectrometry and Scanning Transmission X-ray Microscopy (STXM) combined with soft X-ray Absorption Near Edge Structure (XANES) spectroscopy at the C and $\mathrm{O}$ absorption thresholds to study the chemical environment of organic compounds and hard $\mathrm{x}$-ray XANES to study chemical states of transition metal elements.

Of key importance in analysis of Stardust particles is a non-destructive means of extracting chemical information on both major and trace elemental constituents. We describe a collaborative effort at the Stanford Synchrotron Radiation Laboratory (SSRL) on beamline 6-2 to use micro-focus $\mathrm{x}$-ray beams for the study of meteoritic and cometary materials.

Capabilities: The SSRL beamline 6-2 scanning fluorescence microprobe uses a Kirkpatrick-Baez mirror pair to focus a monochromatic x-ray beam to less than 1 micron in both horizontal and vertical dimensions. The useful energy range spans the $\mathrm{K}$ absorption edges of the transition metals so that both XRF and micro-XANES are possible on scientifically interesting elements such as $\mathrm{Ti}, \mathrm{Fe}$, and Ni. Several examples of recent work with the facility are presented below.

High-resolution XRF mapping. X-ray fluorescence mapping with spatial resolution at the micron level has been achieved on particles embedded in aerogel (Fig. 1). Both major and trace elements down to $\mathrm{Si}$ can be mapped and quantified in this manner. The X-ray probe beam size can be adjusted to scan the sample at different "magnifications" enabling fast coarse maps as well as fine detail on regions of interest. Because the microprobe can nondestructively study particles still embedded in the aerogel capture medium, it is attractive for initial examinations on Stardust and future missions.

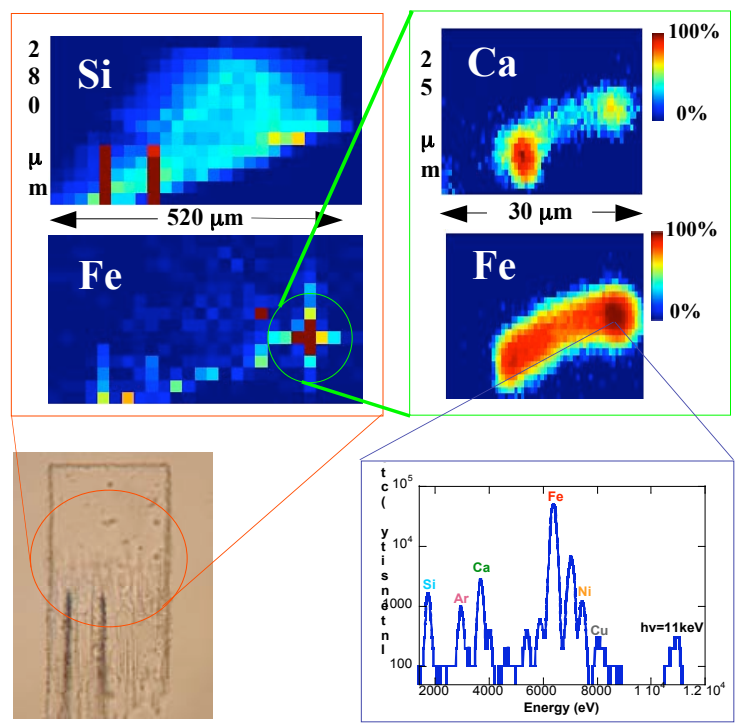

Fig. 1: Clockwise from lower left: Optical micrograph of aerogel keystone, low resolution XRF maps in $\mathrm{Si}$ and $\mathrm{Fe}$ showing locations of the silicon sample mount and the Fe-bearing particles including a grain of Murchison meteorite embedded in the aerogel, high resolution XRF maps of the $\mathrm{Ca}$ and $\mathrm{Fe}$ distributions in the Murchison grain and a log-scale XRF spectrum from the most Fe-rich location in the grain.

As another example of the mapping capability, Figure 2 shows the distribution of $\mathrm{Fe}$ and $\mathrm{Ca}$ fluores- 
cence from a deceleration track of a fragment of A1lende which has been implanted in aerogel using a light gas gun.

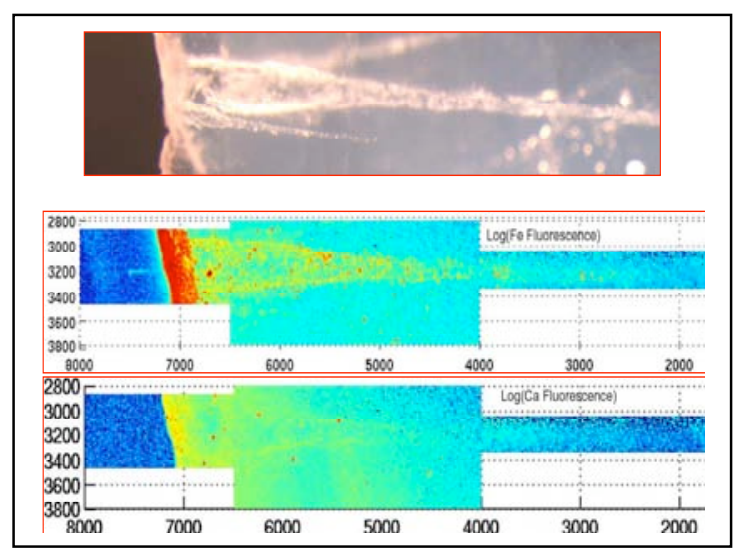

Fig. 2: Maps of a particle track from a gas gun shot of Allende particles. Top: optical image; Middle: Fe fluorescence map; Bottom: Ca fluorescence map. Distances are in microns, so the full $\mathrm{x}$-range is $\sim 6$ $\mathrm{mm}$. Red denotes high signal, blue, low signal.

The similarities between the optical image and the two fluorescence maps are striking. The enhanced Fe fluorescence near the surface of the aerogel is likely due to additional gun debris. This is a twodimensional map, integrating over the volume of the block of aerogel, so low levels of surface contamination are over-emphasized.

Micro-XANES. Prior to the installation of the beamline 6-2 fluorescence microprobe, tests on particles were performed on a total external reflection $\mathrm{x}$ ray fluorescence (TXRF) facility at SSRL [6]. Full particle XANES spectra of a grain of Orgueil meteorite mounted on a Si wafer were collected in grazing incidence geometry at the Fe (Fig. 3) and Ni K edges. Beamline 6-2 has since been rebuilt with improved optics that make spatially-resolved micro-XANES possible at micron resolution with excellent signal-tonoise ratios. This allows mapping of the oxidation state distributions of the majority species within IDPs, micrometeoroids and Stardust comet dust. The low-Z limit for this capability depends on the size of the particle being analyzed since the 1/e escape depth of the characteristic x-rays is energy dependent; however, Ti and higher $\mathrm{Z}$ elements will be readily accessible for most particles. Using Principle Component Analysis methods, mixed valence states can be quantified for each element of interest in a sample using standards containing the relevant valence states. For Fe oxidation states, Sutton et al. [7] have shown that pre-edge peak energy (caused by $1 \mathrm{~s}-3 \mathrm{~d}$ electronic transitions) varies linearly with the proportion of
$\mathrm{Fe}^{3+}$. Wherever possible, such simplifications may be used to speed up analysis.

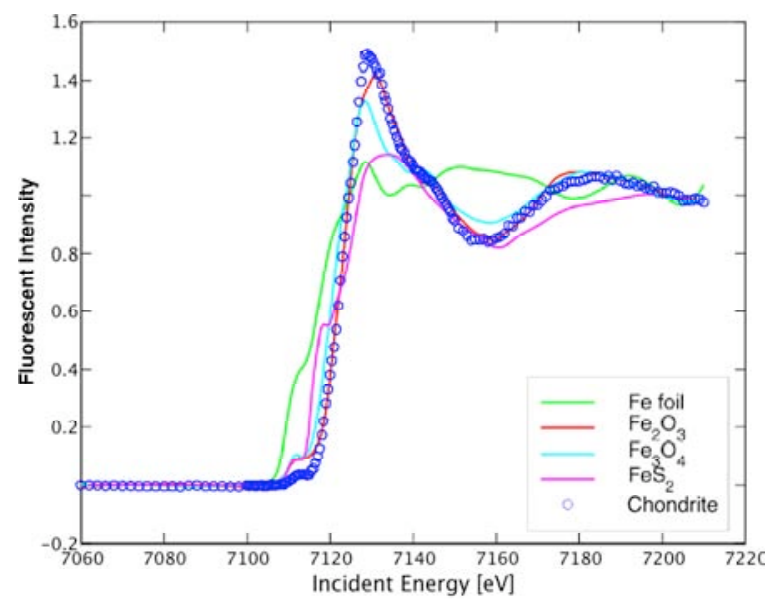

Fig. 3: Example of Fe K-edge XANES previously collected from a grain of Orgueil meteorite (labeled 'Chondrite') in a grazing incidence geometry [from Fig 3 ref 6]. The data are consistent with pure $\mathrm{Fe}_{2} \mathrm{O}_{3}$.

References: [1] Brownlee et al. (2003) JGR, 108, SRD 1-15. [2] Tuzzolino et al. (2004) Science, 304, 1776-1780. [3] Zolensky, M.E. et al. (2000) MAPS, 35, 9-29. [4] Flynn, G.J. et al. (2004) LPS XXXV, Abstract \#1334. [5] Flynn, G.J. et al. (2003) GCA 67, 4791-4806. [6] Brennan S. et al. (2004) Phys Scripta, in print. [7] Sutton S.R. et al. (1995) Rev. Sci. Instrum., 60, 1464-7.

Acknowledgements: This work was performed in part under the auspices of the U.S. Department of Energy, NNSA by the Lawrence Livermore National Laboratory under contract No. W-7405-Eng-48. Portions of this research were carried out at the Stanford Synchrotron Radiation Laboratory, a national user facility operated by Stanford University on behalf of the U.S. Department of Energy, Office of Basic Energy Sciences. 
Graham G.A., Bernas M., Dai Z.R., Bradley J.P., Smith J.B., Weber P.K., Ishii H., Hutcheon I.D. (2005) Recovery of isotopic hotspot in interplanetary dust particles by focused ion beam microscopy: Bridging the gap between NanoSIMS and (S)TEM. Microsc. Microanal. 11(suppl. 2), 1390-1392. UCRL-ABS-21065. 


\title{
Recovery of Isotopic Hotspots in Interplanetary Dust Particles by Focused Ion Beam Microscopy: Bridging the Gap between NanoSIMS and (S)TEM
}

\author{
G.A. Graham,* M. Bernas, ** Z.R, Dai,* J.P. Bradley,* J.B. Smith,* P.K. Weber, * H. Ishii,* and \\ I.D. Hutcheon,*. \\ * Institute of Geophysics \& Planetary Physics, Lawrence Livermore National Laboratory, \\ Livermore, CA 94551 \\ **Application Laboratory, FEI Company, Hillsboro, OR 97124
}

Interplanetary dust particles (IDPs) are a micron-sized $(\sim 5-50 \mu \mathrm{m})$ subset of meteoritic materials derived from comets or asteroids [1]. As IDPs represent some of the most primitive materials available, detailed study of their composition (mineralogy, chemistry and isotopes) gives fundamental insights into early solar system processes [1-3].

The next generation of ion microprobes are now capable of resolving isotopic anomalies of individual grains within IDPs [e.g. 2]. To fully benefit from such observations, it is important to be able to characterize the anomaly in terms of mineralogy or chemistry and its context in terms of the surrounding material within the IDP. In the example shown in Fig. 1, a hydrous IDP (L2047-D23) containing a nitrogen isotopic anomaly has been identified using the NanoSIMS 50 ion microprobe at LLNL [3].

Ultramicrotomy is the traditional method for preparing electron transparent sections of IDPs [4]. While it has been shown that the technique can be utilized to recover sections from IDPs previously prepared for nanoSIMS [2], it does not have the capability of guaranteeing the site-specific recovery of the isotopic anomaly. However, advances in ion beam milling techniques, particularly focused ion beam (FIB) microscopy, have revolutionized the preparation of TEM sections from bulk specimens [5].

An FEI Dual Beam 237 FIB/FESEM instrument was utilized to prepare a TEM section of material containing the anomaly. The region of interest within the IDP was initially coated with a film of $\mathrm{Pt}$ deposited in the FIB first using the electron beam and then using the ion beam. The Pt film or "strap" protects the specimen by reducing excessive superficial ion etching. The Ga+ ion beam operating at $5000 \mathrm{pA}$ current was then used to ablate material on either side of the protective "strap," extracted from the bulk material using an in-situ methodology [e.g. 5], and is subsequentially welded to a (half- cut) TEM grid using Pt. The section is then subjected to further milling at low currents (300pA down to $100 \mathrm{pA})$ and low voltage $(30 \mathrm{keV}$ down to $7 \mathrm{keV})$ to produce a TEM section $\sim 100 \mathrm{~nm}$ in thickness. The dual beam instrument used to prepare the section was fitted with a "flip-stage" that allowed the section to be oriented in a position that is suitable to SEM-STEM imaging. This allows real-time monitoring of the final stages of thinning and preliminary characterization of the final electron transparent section (Fig. 2a). Even at $30 \mathrm{keV}$ it is possible to texturally identify the presence of probable layer-silicates within the section. TEM confirmed the layer-silicates to be serpentine (basal lattice-fringe spacings of $\sim 0.7 \mathrm{~nm}$ ) (Fig. 2b-c).

The FIB recovery of anomalous grains from IDPs enables a new level of integrated analysis between the isotopic and mineralogical studies that will be a key driver in future astromaterials research. 


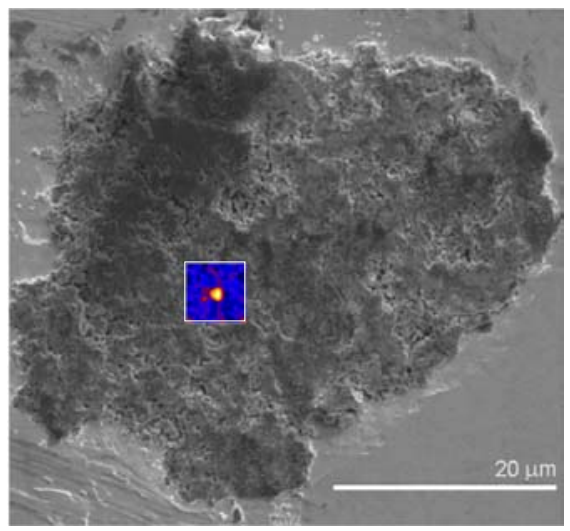

FIG. 1. A secondary electron image of IDP L2047-D23 pressed into gold foil. The insert shows the false color ${ }^{14} \mathrm{~N} /{ }^{15} \mathrm{~N}$ isotopic ratio image of the anomalous grain $(194 \pm 4(2 \sigma)$ compared to $272 \pm 2$ (solar value) for the bulk of the particle) identified within the IDP. The grain is approximately $800 \mathrm{~nm}$ in diameter (data taken from [3]).

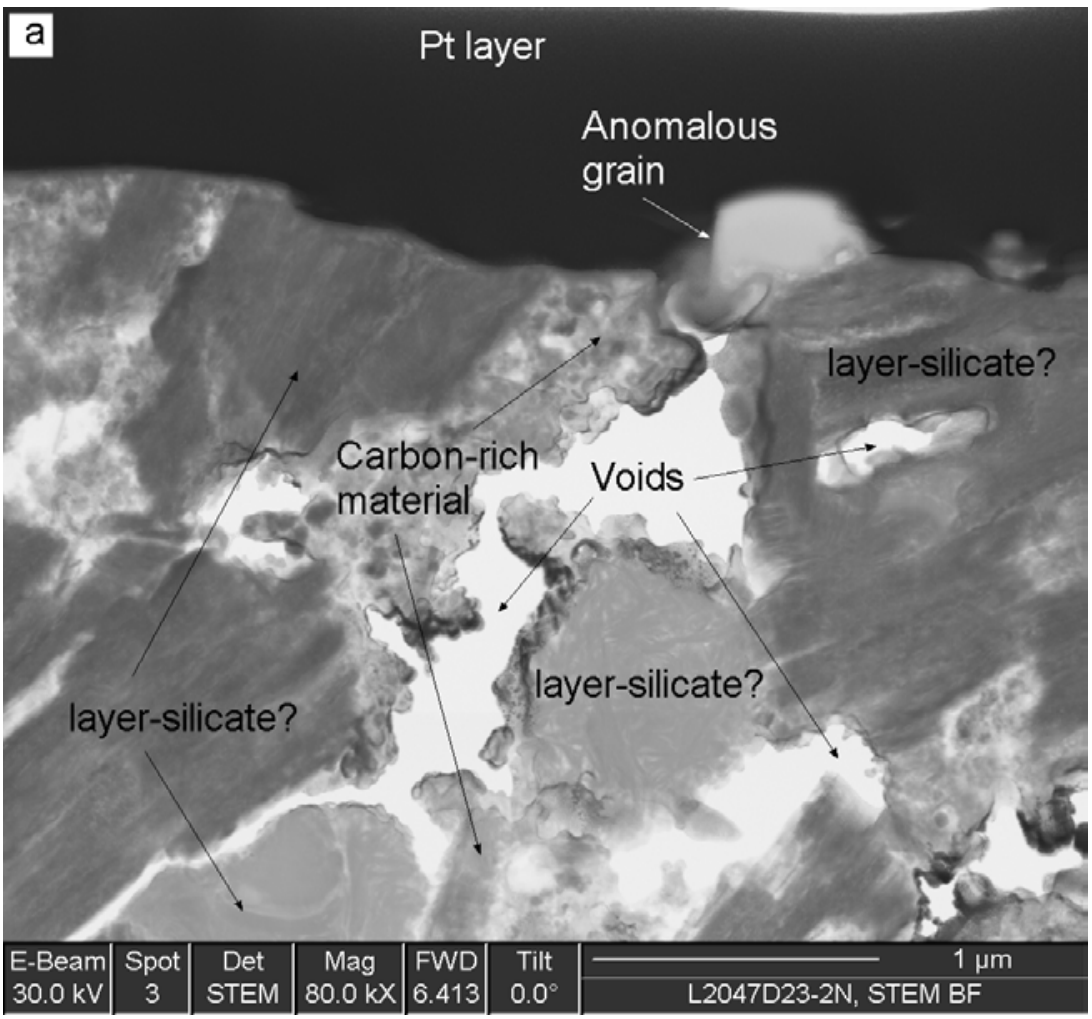

FIG. 2. (a) A 30keV SEM-STEM brightfield image of the extracted section after ion thinning to electron transparency. The fibrous phase is texturally indicative of a layer-silicate, e.g. serpentine or saponite. (b) Acquired lattice fringe image for the layer-silicate phase. (c) SAED pattern for the layer-silicate phase. (b\&c were acquired using CM300 FEGTEM)

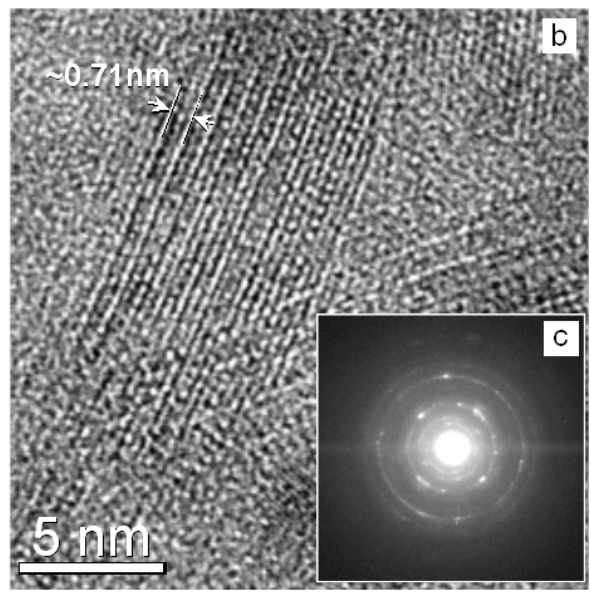

\section{References}

[1] J.P. Bradley, Treatise on Geochemistry, Vol. 1, p689, Elsevier, Amsterdam, 2003.

[2] S. Messenger et al., Science 300 (2000) 105.

[3] J.B. Smith et al., Lunar and Planetary Science XXXVI (2005) abstract \#1003.

[4] J.P. Bradley, Geochimica et Cosmochimica Acta 52 (1988) 889.

[5] L.A. Giannuzzi et al., Introduction to Focused Ion Beams, Springer, New York, 2005.

[6] This work was performed under the auspices of the U.S. Department of Energy, National Nuclear Security Administration by the University of California, Lawrence Livermore National Laboratory under contract No. W7405-Eng-48. The work is supported by NASA grants W-19894, NAG5-10632 and NAG5-10696. 
Floss C., Stadermann F. J., Bradley J., Dai Z.R., Bajt S. and Graham G. A. (2004) Carbon and nitrogen isotopic anomalies in an anhydrous interplanetary dust particle. Science 303, 1355-1358. UCRL-JRNL-201533. 
ality of epitope presentation as the key factor in the rapid and selective differentiation of cells into neurons. An average-sized nanofiber in the network contains an estimated $7.1 \times 10^{14}$ IKVAV epitopes $/ \mathrm{cm}^{2}$. By contrast, closely packed laminin protein molecules in a two-dimensional lattice on a solid substrate have an estimated $7.5 \times 10^{11}$ IKVAV epitopes $/ \mathrm{cm}^{2}(22)$. Thus, the IKVAV nanofibers of the network could amplify the epitope density relative to a laminin monolayer by roughly a factor of $10^{3}(22)$.

The self-assembly of the scaffold studied here can also be triggered by injection of peptide amphiphile solutions into tissue. We injected 10 to $80 \mu \mathrm{l}$ of $1 \mathrm{wt} \%$ peptide amphiphile solutions into freshly enucleated rat eye preparations and in vivo into rat spinal cords following a laminectomy to expose the cord (22). Thus, these peptide amphiphile solutions can indeed be transformed into a solid scaffold upon contact with tissues. This process localizes the network in tissue and prevents passive diffusion of the molecules away from the epicenter of an injection site. Furthermore, it is known that animals survive for prolonged periods after injections of the peptide amphiphile solutions into the spinal cord, a finding of relevance to the present study.

\section{References and Notes}

1. R. Langer, J. P. Vacanti, Science 260, 920 (1993).

2. A. Lendlein, R. Langer, Science 296, 1673 (2002).

3. Y. D. Teng et al., Proc. Natl. Acad. Sci. U.S.A. 99, 3024 (2002).

4. L. Lu et al., Biomaterials 21, 1837 (2000).

5. L. E. Niklason., Science 284, 489 (1999).

6. S. Nehrer et al., J. Biomed. Mater. Res. 38, 95 (1997)

7. A. Atala et al., J. Urol. 150, 745 (1993).

8. H. L. Wald et al., Biomaterials 14, 270 (1993)

9. I. V. Yannas, J. F. Burke, D. P. Orgill, E. M. Skrabut, Science 215, 174 (1982).

10. D. J. Mooney et al., Biomaterials 17, 1417 (1996)

11. A. G. Mikos, M. D. Lyman, L. E. Freed, R. Langer Biomaterials 15, 55 (1994).

12. E. Lavik, Y. D. Teng, E. Snyder, R. Langer, Methods Mol. Biol. 198, 89 (2002).

13. W. C. Hsu, M. H. Spilker, I. V. Yannas, P. A. Rubin Invest. Ophthalmol. Vis. Sci. 41, 2404 (2000).

14. L. J. Chamberlain, I. V. Yannas, H. P. Hsu, G. R. Strichartz, M. Spector, J. Neurosci. Res. 60, 666 (2000).

15. C. E. Butler, I. V. Yannas, C. C. Compton, C. A. Correia, D. P. Orgill, Br. J. Plast. Surg. 52, 127 (1999).

16. D. P. Orgill et al., Plast. Reconstr. Surg. 102, 423 (1998).

17. S. C. Chang et al., J. Biomed. Mater. Res. 55, 503 (2001).

18. A. Atala et al., J. Urol. 150, 745 (1993)

19. F. Lim, A. M. Sun, Science 210, 908 (1980).

20. G. Hortelano, A. Al-Hendy, F. A. Ofosu, P. L. Chang, Blood 87, 5095 (1996).

21. W. Xu, L. Liu, I. G. Charles, FASEB J. 16, 213 (2002).

22. Materials and methods are available as supporting material on Science Online.

23. H. Okano, J. Neurosci. Res. 69, 698 (2002).

24. A. Storch, J. Schwarz, Curr. Opin. Invest. Drugs 3, 774 (2002).

25. M. F. Mehler, J. A. Kessler, Arch. Neurol. 56, 780 (1999).

26. D. W. Pincus, R. R. Goodman, R. A. Fraser, M. Nedergaard, S. A. Goldman, Neurosurgery 42, 858 (1998).

27. L. Kam, W. Shain, J. N. Turner, R. Bizios, Biomaterials 22, 1049 (2001)

28. M. Matsuzawa, F. F. Weight, R. S. Potember, P. Liesi, Int. J. Dev. Neurosci. 14, 283 (1996).

29. S. K. Powell et al., J. Neurosci. Res. 61, 302 (2000).
30. T. Cornish, D. W. Branch, B. C. Wheeler, J. T. Campanelli, Mol. Cell. Neurosci. 20, 140 (2002)

31. J. C. Chang, G. J. Brewer, B. C. Wheeler, Biosens. Bioelectron. 16, 527 (2001).

32. B. C. Wheeler, J. M. Corey, G. J. Brewer, D. W. Branch, J. Biomech. Eng. 121, 73 (1999).

33. L. Lauer, A. Vogt, C. K. Yeung, W. Knoll, A. Offenhausser, Biomaterials 23, 3123 (2002).

34. P. Thiebaud, L. Lauer, W. Knoll, A. Offenhausser, Biosens. Bioelectron. 17, 87 (2002).

35. C. K. Yeung, L. Lauer, A. Offenhausser, W. Knoll, Neurosci. Lett. 301, 147 (2001).

36. The EQS peptide sequence has no known physiological signaling function but has a charge distribution that allows nanofiber self-assembly.

37. J. D. Hartgerink, E. Beniash, S. I. Stupp, Science 294, 1684 (2001).

38. J. D. Hartgerink, E. Beniash, S. I. Stupp, Proc. Natl. Acad. Sci. U.S.A. 99, 5133 (2002).

39. In vitro self-assembly was induced by addition of Dulbecco's minimum essential medium (DMEM), DMEM/F12, and modifications thereof, as well as by addition of cerebral spinal fluid.

40. Cell viability and toxicity was assessed using Molecular Probes Live/Dead assay (22).

41. G. A. Silva et al., data not shown.

42. G. Zhu, M. F. Mehler, P. C. Mabie, J. A. Kessler, J. Neurosci. Res. 59, 312 (2000)

43. A. G. Rabchevsky, G. M. Smith, Arch. Neurol. 58, 721 (2001).

44. Z. J. Chen, Y. Ughrin, J. M. Levine, Mol. Cell. Neurosci. 20, 125 (2002).

45. S. Costa et al., Glia 37, 105 (2002)

46. F. H. Gage, J. Ray, L. J. Fisher, Annu. Rev. Neurosci. 18, 159 (1995).

47. M. Parmar, C. Skogh, A. Bjorklund, K. Campbell, Mol. Cell. Neurosci. 21, 645 (2002)

48. S. Wu et al., J. Neurosci. Res. 72, 343 (2003).
49. E. Alsberg, K. W. Anderson, A. Albeiruti, J. A. Rowley, D. J. Mooney, Proc. Natl. Acad. Sci. U.S.A. 99, 12025 (2002).

50. L. Canaple, A. Rehor, D. Hunkeler, J. Biomater. Sci. Polym. Ed. 13, 783 (2002).

51. S. C. Chang et al., J. Biomed. Mater. Res. 55, 503 (2001).

52. J. J. Marler et al., Plast. Reconstr. Surg. 105, 2049 (2000).

53. J. A. Rowley, D. J. Mooney, J. Biomed. Mater. Res. 60, 217 (2002)

54. For 2D IKVAV-PA cell experiments, we first coated cover slips with PDL to ensure that the negatively charged IKVAV-PA nanofibers would adhere to the surface, and then placed $300 \mathrm{ml}$ of $1 \mathrm{wt} \%$ aqueous solutions of the PA on surfaces and allowed them to dry overnight in a fume hood. We prepared the IKVAV peptide cover slips by spin-coating the peptide on the surface. The next day, all coated plates were washed three times with distilled water to remove any material not strongly adsorbed to the surface.

55. This material is based on work supported by the U.S. Department of Energy (grant DE-FG02-00ER45810/ A001), NIH (grants NS20778, NS20013, and NS34758), and NSF (DMR-010-8342). Any opinions, findings, and conclusions or recommendations expressed in this work are those of the authors and do not necessarily reflect the views of these agencies.

Supporting Online Material

www.sciencemag.org/cgi/content/full/1093783/DC1

Materials and Methods

Fig. S1

References and Notes

18 November 2003; accepted 13 January 2004 Published online 22 January 2004;

10.1126 /science. 1093783

Include this information when citing this paper.

\title{
Carbon and Nitrogen Isotopic Anomalies in an Anhydrous Interplanetary Dust Particle
}

\author{
Christine Floss, ${ }^{1 *}$ Frank J. Stadermann, ${ }^{1}$ John Bradley, ${ }^{2}$ \\ Zu Rong Dai, ${ }^{2}$ Saša Bajt, ${ }^{2}$ Giles Graham ${ }^{2}$
}

Because hydrogen and nitrogen isotopic anomalies in interplanetary dust particles have been associated with carbonaceous material, the lack of similar anomalies in carbon has been a major conundrum. We report here the presence of a ${ }^{13} \mathrm{C}$ depletion associated with a ${ }^{15} \mathrm{~N}$ enrichment in an anhydrous interplanetary dust particle. Our observations suggest that the anomalies are carried by heteroatomic organic compounds. Theoretical models indicate that lowtemperature formation of organic compounds in cold interstellar molecular clouds can produce carbon and nitrogen fractionations, but it remains to be seen whether the specific effects observed here can be reproduced.

Interstellar molecular clouds are the principal formation sites of organic matter in the Milky Way galaxy. A variety of simple molecules, such as $\mathrm{CH}_{4}, \mathrm{CH}_{3} \mathrm{OH}$, and $\mathrm{H}_{2} \mathrm{CO}$, are produced in dense cold (10 to $30 \mathrm{~K}$ ) clouds (1). At such low temperatures, where the differ-

${ }^{1}$ Laboratory for Space Sciences, Washington University, St. Louis, MO 63130, USA. ${ }^{2}$ Institute for Geophysics and Planetary Physics, Lawrence Livermore National Laboratory, Livermore, CA 94550, USA

*To whom correspondence should be addressed. Email: floss@wustl.edu ence in chemical binding energy exceeds thermal energy, mass fractionation produces molecules with isotopic ratios that can be anomalous relative to terrestrial values $(1-3)$. Such anomalous ratios potentially provide a fingerprint for abiotic interstellar organic matter that was incorporated into the solar system and survives today in cosmically primitive materials such as interplanetary dust particles (IDPs)

IDPs collected in Earth's stratosphere are complex assemblages of primitive solar system material and carry various isotopic 
anomalies $(2,4,5)$. Deuterium enhancements result from extreme chemical fractionation in cold molecular clouds $(6)$, and $\mathrm{D} / \mathrm{H}$ ratios in some IDPs approach the values observed in interstellar molecules (7), suggesting the intact survival of some molecular cloud material. Nitrogen isotopic anomalies, in the form of ${ }^{15} \mathrm{~N}$ enrichments, are also common and are postulated to result from low-temperature interstellar chemistry $(2,8)$. This origin is complicated because $\mathrm{N}$ isotopic fractionation has not been observed in the interstellar medium and because anomalous $\mathrm{N}$ can also have a nucleosynthetic origin. However, recent work shows that chemical reactions in dense molecular gases can produce elevated ${ }^{15} \mathrm{~N} /{ }^{14} \mathrm{~N}$ ratios $(9,10)$, although the maximum enhancements fall short of observed ${ }^{15} \mathrm{~N}$ enrichments in IDPs $(11,12)$. Organic compounds appear to be the source of many of the $\mathrm{D}$ and ${ }^{15} \mathrm{~N}$ enrichments $(13-15)$; it is puzzling, therefore, that $\mathrm{C}$ isotopic anomalies have not been observed, despite numerous measurements (5).

We carried out simultaneous $\mathrm{C}$ and $\mathrm{N}$ isotopic imaging measurements of an anhydrous noncluster particle (L2036-G16), using the NanoSIMS, a new generation secondary ion mass spectrometer that allows isotopic imaging at a spatial scale of $100 \mathrm{~nm}$. The particle, nicknamed Benavente (16), was pressed into a high-purity Au substrate along with isotopic standards, which were measured together with the IDP on the same sample mount (17). The bulk $\mathrm{C}$ isotopic composition of Benavente is normal, with a ${ }^{12} \mathrm{C} /$ ${ }^{13} \mathrm{C}$ ratio of $89.3 \pm 1.0\left(\delta^{13} \mathrm{C}=+8 \pm 11 \%\right.$ ), but the IDP is enriched in ${ }^{15} \mathrm{~N}$, with an average ${ }^{14} \mathrm{~N} /{ }^{15} \mathrm{~N}$ ratio of $224.3 \pm 1.7\left(\delta^{15} \mathrm{~N}=\right.$ $+213 \pm 9 \%$ ). Benavente also contains a large $\left(0.6 \times 1.8 \mu \mathrm{m}^{2}\right)$ region that is strongly enriched in ${ }^{15} \mathrm{~N}\left({ }^{14} \mathrm{~N} /{ }^{15} \mathrm{~N}=119.8 \pm 1.3\right.$; $\delta^{15} \mathrm{~N}=+1270 \pm 25 \%$ ) and depleted in ${ }^{13} \mathrm{C}$ $\left({ }^{12} \mathrm{C} /{ }^{13} \mathrm{C}=96.6 \pm 1.3 ; \delta^{13} \mathrm{C}=-70 \pm 13 \%\right)$ (Fig. 1) (18). Although the ${ }^{13} \mathrm{C}$ depletion is not great, its importance is emphasized by the large size of the region and its association with the ${ }^{15} \mathrm{~N}$ enrichment (Fig. 2) (19). Furthermore, both anomalies are consistently present in the 25 successively measured layers. Several studies (20-22) indicate a solar N isotopic composition that is $\sim 30 \%$ lighter than the terrestrial one. Thus, the ${ }^{15} \mathrm{~N}$ enrichments in Benavente relative to solar composition may be considerably higher than the values noted here. Moreover, recent work (23) suggests that solar C may be $\sim 10 \%$ lighter than terrestrial $\mathrm{C}$, a value that is similar to the magnitude of the ${ }^{13} \mathrm{C}$ depletion in our anomalous region.

In addition to isotopic information, our analysis provides clues to the nature of the carrier phase(s) of the $\mathrm{C}$ and $\mathrm{N}$ anomaly in Benavente. The anomalous region has a high-

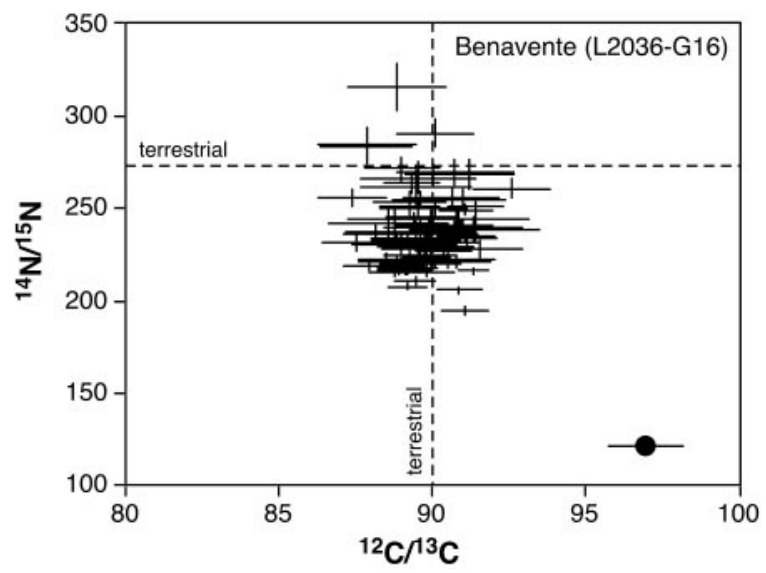

Fig. 1. Isotopic composition of the anomalous region compared with similar-sized areas of Benavente. Nitrogen isotopic compositions spread toward subterrestrial ratios in the "bulk" of the particle, indicating an overall enrichment in ${ }^{15} \mathrm{~N}$.
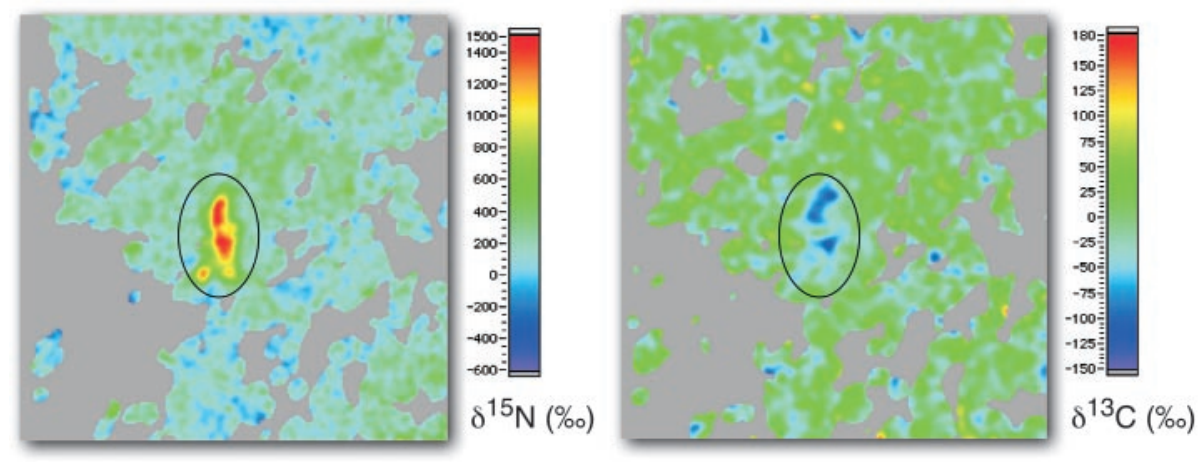

Fig. 2. $\delta^{15} \mathrm{~N}$ (left) and $\delta^{13} \mathrm{C}$ (right) images of Benavente showing the ${ }^{15} \mathrm{~N}$-enriched and ${ }^{13} \mathrm{C}$ depleted anomalous region. Field of view is $10 \mu \mathrm{m}$.

er $\mathrm{C}^{-}$yield than most of the IDP and a $\mathrm{CN}^{-} / \mathrm{C}^{-}$ratio of $\sim 0.9$, suggesting that the $\mathrm{C}$ and $\mathrm{N}$ anomalies are carried by organic matter. If this material is carbonaceous, the calibration curve of $\mathrm{CN}^{-} / \mathrm{C}^{-}$ratios versus $\mathrm{N}$ concentrations in organic material established by (24) suggests a $N$ concentration of $\sim 3.0 \pm$ 1.5 weight $\%$ (wt $\%$ ). This is at the upper end of the range of $\mathrm{N}$ concentrations of insoluble organic matter in carbonaceous chondrites (15) and falls within the field observed for CHON grains from comet Halley (25). Recent measurements of the ${ }^{14} \mathrm{~N} /{ }^{15} \mathrm{~N}$ ratios of the $\mathrm{CN}$ radical in the coma of two comets give a value of $140 \pm 30(26)$, within errors the same as that of the ${ }^{14} \mathrm{~N} /{ }^{15} \mathrm{~N}$ ratio of our anomalous region.

Using a focused ion beam (FIB) technique (27), we extracted a section $(\sim 5$ by 1 by $0.1 \mu \mathrm{m}$ ) from an area about $5 \mu \mathrm{m}$ from the anomalous region. This area has lower $\mathrm{C}^{-}$and $\mathrm{CN}^{-}$signals than the anomalous region, and $\mathrm{C}$ and $\mathrm{N}$ isotopic compositions similar to the bulk IDP. Using a transmission electron microscope (TEM), we employed energy-filtered imaging to investigate the mineralogy of the section (17). The region is rich in GEMS (glass with embedded metal and sulfides) (28) and also contains forsterite $\left(\mathrm{Mg}_{2} \mathrm{SiO}_{4}\right)$, iron-rich sulfides (pyrrhotite, $\mathrm{Fe}_{1-\mathrm{x}} \mathrm{S}$ ), and abundant amorphous carbonaceous material that is clumped into distinct regions and coats some grains, such as the GEMS.

After the TEM investigation, we mapped the $\mathrm{C}$ and $\mathrm{N}$ isotopic compositions in the FIB slice with the NanoSIMS and reconfirmed the ${ }^{15} \mathrm{~N}$ enrichment of $\sim 200$ $\%$ observed in the bulk IDP. Moreover, we found a $250-\mathrm{nm}$ region with a strong ${ }^{15} \mathrm{~N}$ enrichment of $+1110 \pm 98 \%$ that was not seen in the original NanoSIMS measurement because it is located below the original surface of the IDP. Spatial correlation of this anomaly with the TEM images (Fig. 3) shows that it consists of amorphous carbonaceous material immediately surrounded by GEMS and other silicates. Infrared (IR) spectra (17) show a prominent C-H stretch feature at $\sim 3.4 \mu \mathrm{m}$ (Fig. 4 ), similar to that observed in meteoritic kerogen (4). The positions of the bands within the feature are consistent with those of aliphatic hydrocarbons, confirming the organic nature of carbonaceous material. Aromatic hydrocarbons are likely to be present too, but are probably dominated by the strong resonance of the aliphatic $\mathrm{C}-\mathrm{H}$ feature. Nitrogen abundances in the FIB section are low (1 to 2 wt $\%$, based on electron energy loss spectroscopy), but the $\mathrm{N}$ is associated with the carbonaceous material and, thus, has an organic origin. No other elements are associated with the $\mathrm{N}$ 

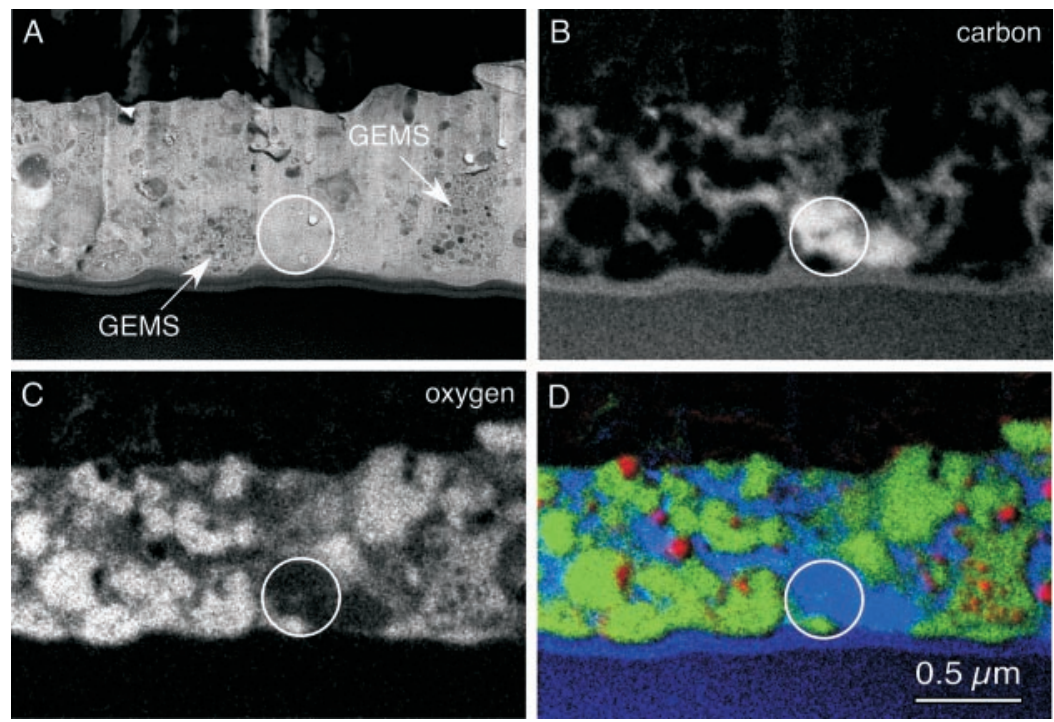

Fig. 3. FIB section extracted from Benavente showing, in the circle, the ${ }^{15} \mathrm{~N}$-rich region. (A) Bright-field TEM image (300 keV). GEMS, glass with embedded metal and sulfides. (B) Energyfiltered carbon jump-ratio image. (C) Energy-filtered oxygen jump-ratio image. (D) Composite image showing silicates (green), carbonaceous material (blue), and iron or iron sulfides (red).

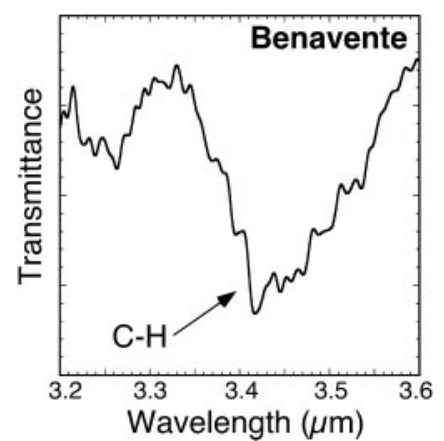

Fig. 4. IR spectrum of the Benavente FIB section, showing the $\mathrm{C}-\mathrm{H}$ stretch feature at $3.4 \mu \mathrm{m}$ indicative of hydrocarbons.

that might indicate the presence of a different carrier phase. The ${ }^{15} \mathrm{~N}$-rich region in the FIB slice is not ${ }^{13} \mathrm{C}$-depleted (probably because of the small size of the area, leading to dilution effects from surrounding isotopically normal C), but the similarity of the magnitude of the $\mathrm{N}$ anomaly to that of the $\mathrm{C}$ - and $\mathrm{N}$-anomalous region (and the proximity of the FIB slice to the original anomalous area) suggests that the same type of amorphous carbon is the carrier of the correlated $\mathrm{C}$ and $\mathrm{N}$ anomaly. Although presolar graphite in meteorites is ${ }^{15} \mathrm{~N}$ enriched and ${ }^{13} \mathrm{C}$-depleted (29), it is unlikely to be the carrier phase of the $\mathrm{C}$ and $\mathrm{N}$ anomaly in Benavente: Our TEM and spectroscopic work shows that only amorphous carbon is present in this IDP. Moreover, in contrast to carbonaceous chondrites, graphitic carbon is conspicuously absent from chondritic IDPs $(4,30)$.

Without $\mathrm{C}$ isotopic anomalies, it is not possible to distinguish whether the carbonaceous material in IDPs is itself presolar or simply a more "recent" (e.g., solar system) host substrate for presolar D and ${ }^{15} \mathrm{~N}-$ enriched species. Previous studies have suggested that some IDPs contain carbon compounds with heteroatoms (e.g., $\mathrm{N}$ in $-\mathrm{CN}$ attached to aromatic chromophores) (31), but without correlated $\mathrm{C}$ and $\mathrm{N}$ isotopic data, the origin of such molecules has remained uncertain $(13,31,32)$. Our observation of associated $\mathrm{C}$ and $\mathrm{N}$ anomalies establishes that IDPs contain heteroatomic organic compounds of presolar interstellar origin that are more complex than the simple deuterated compounds implied by earlier measurements (7). During its prebiotic period, Earth may have accreted as much as a centimeter of abiotic carbonaceous matter every million years, much of it settling to the surface within small ( $<25 \mu \mathrm{m}$ diameter), high-surfacearea IDPs (33-35). This constant flux of particulate organic matter continues to be delivered to the surfaces of terrestrial planets today and includes interstellar molecules such as those found in Benavente.

Gas-phase reactions are expected to produce $\mathrm{C}$ isotopic fractionations, but different processes produce fractionation effects in opposite directions $(1,36-38)$. Thus, it has been suggested that the lack of $\mathrm{C}$ isotopic anomalies in IDPs is due to the existence of multiple reaction pathways that cancel out any anomalies produced $(1,38)$. Others have suggested that isotopic fractionation in $\mathrm{C}$ is inhibited through the condensation of $\mathrm{CO}$ onto grain surfaces and its participation in grain chemistry $(2,5)$. Our observation of a ${ }^{13} \mathrm{C}$ depletion associated with a ${ }^{15} \mathrm{~N}$ enrichment in Benavente shows that $\mathrm{C}$ isotopic fractionation does occur and requires processes that can produce both effects in the same material.
Gas-phase ion-molecule reactions can enhance the ${ }^{12} \mathrm{C} /{ }^{13} \mathrm{C}$ ratios of organic species $(36,37)$ to the level observed in Benavente, but whether these reactions also result in depleted ${ }^{14} \mathrm{~N} /{ }^{15} \mathrm{~N}$ ratios has not been studied. Low-temperature interstellar chemistry as the source of the ${ }^{15} \mathrm{~N}$ enrichments seen in IDPs has only recently been investigated theoretically. A study of ion-molecule exchange reactions involving the most abundant $\mathrm{N}$-bearing species in interstellar clouds (9) indicated a maximum enhancement in ${ }^{15} \mathrm{~N}$ of $+250 \%$. Another recent model, investigating $\mathrm{NH}_{3}$ formation in dense molecular clouds, suggests a maximum enrichment of $+800 \%$ (10). These models are consistent with the modest enrichment in ${ }^{15} \mathrm{~N}$ seen in the bulk IDP but fall short of the values needed to account for the $+1270 \%$ enrichment in ${ }^{15} \mathrm{~N}$ observed in the anomalous region. Moreover, it is not clear whether ${ }^{15} \mathrm{~N}$-rich ammonia can pass on its anomalous $\mathrm{N}$ to the organic hosts thought to be responsible for $\mathrm{N}$ isotopic anomalies in IDPs. If future investigations of interstellar chemistry cannot account for the $\mathrm{C}$ and $\mathrm{N}$ isotopic fractionations observed in IDPs, circumstellar origins may need to be considered.

\section{References and Notes}

1. A. G. G. M. Tielens, in Astrophysical Implications of the Laboratory Study of Presolar Materials, T. J. Bernatowicz, E. Zinner, Eds. (AIP Conference Proceedings 402, American Institute of Physics, Woodbury, NY, 1998), pp. 523-544.

2. S. Messenger, R. M. Walker, in Astrophysical Implications of the Laboratory Study of Presolar Materials, T. J. Bernatowicz, E. Zinner, Eds. (AIP Conference Proceedings 402, American Institute of Physics, Woodbury, NY, 1998), pp. 545-564.

3. $\mathrm{H} / \mathrm{D}=6666,{ }^{12} \mathrm{C} /{ }^{13} \mathrm{C}=90$, and ${ }^{14} \mathrm{~N} /{ }^{15} \mathrm{~N}=272$. Isotopic anomalies are often expressed as delta values, representing the deviation of the measured isotopic ratio from the terrestrial standard in parts per thousand $(\% \circ)$

4. J. P. Bradley, in Meteorites, Planets, and Comets, A. M. Davis, Ed. (Vol. 1, Treatise on Geochemistry, H. D. Holland, K. K. Turekian, Exec. Eds., Elsevier Science, 2003), pp. 689-711.

5. S. Messenger, F. J. Stadermann, C. Floss, L. R. Nittler, S. Mukhopadhyay, Space Sci. Rev. 106, 155 (2003).

6. T. J. Millar, A. Bennett, E. Herbst, Astrophys. J. 340, 906 (1989).

7. S. Messenger, Nature 404, 968 (2000).

8. C. M. O'D. Alexander et al., Meteorit. Planet. Sci. 33, 603 (1998).

9. R. Terzieva, E. Herbst, Mon. Not. R. Astron. Soc. 317, $563(2000)$

10. S. B. Charnley, S. D. Rodgers, Astrophys. J. 569, L133 (2002).

11. C. Floss, F. J. Stadermann, Lunar Planet. Sci. 33, 1350 (2002).

12. C. Floss, F. J. Stadermann, Lunar Planet. Sci. 34, 1238 (2003).

13. L. P. Keller, S. Messenger, M. Miller, K. L. Thomas, Lunar Planet. Sci. 28, 707 (1997).

14. J. Aléon, F. Robert, M. Chaussidon, B. Marty, C. Engrand, Lunar Planet. Sci. 33, 1397 (2002).

15. J. Aléon et al., Lunar Planet. Sci. 34, 1308 (2003).

16. Jacinto Benavente won the Nobel Prize for Literature in 1922 for his contributions to Spanish drama.

17. Materials and methods are available as supporting material on Science Online.

18. The image was divided into 94 randomly generated regions similar in size and shape to the anomalous 
region, with analytical errors $(1 \sigma)$ of $4 \%$ or less for both ${ }^{14} \mathrm{~N} /{ }^{15} \mathrm{~N}$ and ${ }^{12} \mathrm{C} /{ }^{13} \mathrm{C}$.

19. While the anomalous regions in $\mathrm{C}$ and $\mathrm{N}$ have slightly different shapes, the differences are at the limit of the lateral resolution of these smoothed images and are, therefore, of limited importance. However, all extracted $\mathrm{C}$ and $\mathrm{N}$ isotopic ratios (e.g., Fig. 1) refer to identical regions of interest and are, thus, spatially correlated.

20. Th. Fouchet et al., Icarus 143, 223 (2000).

21. K. Hashizume, M. Chaussidon, B. Marty, F. Robert, Science 290, 1142 (2000).

22. T. Owen, P. R. Mahaffy, H. B. Nieman, S. Atreya, M. Wong, Astrophys. J. 553, L77 (2001).

23. K. Hashizume, M. Chaussidon, B. Marty, K. Terada, Astrophys. J. 600, 480 (2004).

24. J. Aléon, F. Robert, M. Chaussidon, B. Marty, Geochim. Cosmochim. Acta 67, 3773 (2003).

25. M. N. Fomenkova, S. Chang, L. M. Mukhin, Geochim. Cosmochim. Acta 58, 4503 (1994).

26. C. Arpigny et al., Science 301, 1522 (2003).

27. P. J. Heaney, E. P. Vicenzi, L. A. Giannuzzi, K. J. T. Livi, Am. Mineral. 86, 1094 (2001).

28. J. P. Bradley, Science 265, 925 (1994).
29. P. Hoppe, S. Amari, E. Zinner, R. S. Lewis, Geochim Cosmochim. Acta 59, 4029 (1995).

30. L. P. Keller, S. Messenger, J. P. Bradley, J. Geophys. Res. 105, 10397 (2000).

31. S. J. Clemett, C. R. Maechling, R. N. Zare, P. D. Swan, R. M. Walker, Science 262, 721 (1993).

32. G. J. Flynn, L. P. Keller, D. Joswiak, D. E. Brownlee, Lunar Planet. Sci. 33, 1320 (2002).

33. D. E. Brownlee, in Analysis of Interplanetary Dust, M. E. Zolensky, T. L. Wilson, F. Y. M. Rietmeijer, G. J. Flynn, Eds. (AIP Conference Proceedings 310, American Institute of Physics, Woodbury, NY, 1994), pp. 5-8.

34. D. E. Brownlee, D. J. Joswik, M. E. Kress, S. Taylor, J. Bradley, Lunar Planet. Sci. 33, 1786 (2002).

35. J. P. Bradley, D. E. Brownlee, Science 231, 1542 (1986).

36. W. D. Langer, T. E. Graedel, M. A. Frerking, P. B. Armentrout, Astrophys. J. 277, 581 (1984).

37. W. D. Langer, T. E. Graedel, Astrophys. J. 69, 241 (1989).

38. S. A. Sandford, M. P. Bernstein, J. P. Dworkin, Meteorit. Planet. Sci. 36, 1117 (2001).

39. This work was supported by NASA grants NAG5-
13467 to C.F., and NAG5-10623 and NAG5-10696 to J.B.. The work by J.B., Z.D., S.B., and G.G. was performed under the auspices of the U.S. Department of Energy, National Nuclear Security Administration by the University of California, Lawrence Livermore National Laboratory (LLNL) under contract No. W-7405Eng-48. Department of Energy (DOE) contract DEAC03-76SF00098 supported electron microscopy performed at the National Center for Electron Microscopy at Lawrence Berkeley National Laboratory (LBNL) and infrared spectroscopy performed at the Advanced Light Source (ALS) at LBNL. We thank T. Smolar of Washington University for NanoSIMS maintenance and support, M. Martin and ALS personnel for providing the ALS beamline, and $\mathrm{K}$. Moore at LLNL for assistance with energy-filtered imaging.

Supporting Online Material www.sciencemag.org/cgi/content/full/303/5662/1355/ DC1 Materials and Methods References

4 November 2003; accepted 26 January 2004

\section{Legionella Effectors That Promote Nonlytic Release from Protozoa}

\author{
John Chen, ${ }^{1}$ Karim Suwwan de Felipe, ${ }^{2}$ Margaret Clarke, ${ }^{3}$ \\ Hao Lu, ${ }^{3}$ O. Roger Anderson, ${ }^{4}$ Gil Segal, ${ }^{5}$ Howard A. Shuman ${ }^{1 *}$
}

\begin{abstract}
Legionella pneumophila, the bacterial agent of legionnaires' disease, replicates intracellularly within a specialized vacuole of mammalian and protozoan host cells. Little is known about the specialized vacuole except that the $\mathrm{Icm} /$ Dot type IV secretion system is essential for its formation and maintenance. The Legionella genome database contains two open reading frames encoding polypeptides (LepA and LepB) with predicted coiled-coil regions and weak homology to SNAREs; these are delivered to host cells by an $\mathrm{lcm} /$ Dot-dependent mechanism. Analysis of mutant strains suggests that the Lep proteins may enable the Legionella to commandeer a protozoan exocytic pathway for dissemination of the pathogen.
\end{abstract}

Several intracellular pathogens including $L e$ gionella pneumophila, Mycobacterium avium (1), Chlamydia spp. (2), and Francisella tularensis (3) are able to replicate within protozoan trophozoites. Thus, free-living amoebae may serve as a significant reservoir for pathogens in the environment, perhaps even as a "training environment" for the selection of virulence-related traits in these pathogens (4). L. pneumophila, the causative agent of legionnaires' disease, is frequently detected

${ }^{1}$ Department of Microbiology, and ${ }^{2}$ Integrated Program in Cellular, Molecular and Biophysical Studies, College of Physicians and Surgeons, Columbia University, 701 West 168th Street, New York, NY 10032, USA. ${ }^{3}$ Program in Molecular and Cell Biology, Oklahoma Medical Research Foundation, 825 N.E. 13th Street, Oklahoma City, OK 73104-5046, USA. ${ }^{4}$ Department of Biology, Lamont-Doherty Earth Observatory, Columbia University, 61 Route 9W, Palisades, NY 10964-1000, USA. ${ }^{5}$ Department of Molecular Microbiology and Biotechnology, George S. Wise Faculty of Life Sciences, Tel-Aviv University, Ramat-Aviv, Tel-Aviv 69978, Israel.

*To whom correspondence should be addressed. Email: has7@columbia.edu in association with Hartmanella vermiformis at the sources of infection during outbreaks (5). Under experimental conditions, L. pneumophila can multiply within and kill a variety of phylogenetically unrelated protozoa ranging from Acanthamoeba castellani to the genetically well characterized social amoeba Dictyostelium discoideum $(6,7)$.

Intracellular pathogens have evolved three distinct strategies for surviving phagosomelysosome fusion. Two of these mechanisms, tolerance of the toxic environment and escape from the phago-lysosome into the cytosol, are used by a variety of pathogens such as Salmonella enterica and Listeria monocytogenes, respectively. L. pneumophila and several other prokaryotic and eukaryotic intracellular pathogens use a third strategyprevention of phagosome-lysosome fusion. After uptake of L. pneumophila by macrophages or protozoa, the bacteria are found within a specialized vacuole that does not fuse with lysosomes and does not acidify $(8,9)$; this allows replication to proceed. The specialized vacuole associates with en- doplasmic reticulum (ER)-derived secretory vesicles (10), mitochondria, and rough ER $(11,12)$ and, near the end of the replicative cycle, acquires late endosomal markers (13). These observations strongly suggest that the bacteria play an active and continuous role in modulating organelle trafficking events from within the confines of their specialized vacuole.

A group of 24 genes called icm (intracellular multiplication) (14) or dot (defect in organelle trafficking) (15) are required for intracellular multiplication of $L$. pneumophila. Sequence similarity between several Icm/Dot proteins and those of the conjugative system of IncI plasmids colIb-9 and R64 (16) indicates that the $\mathrm{Icm} /$ Dot proteins form a type IV secretion system (TFSS) that delivers effectors to host cells. However, $L$. pneumophila mutants lacking the previously identified RalF or LidA effectors do not display obvious defects in organelle trafficking or intracellular replication $(17,18)$. Because direct biochemical observation of infected cells has not led to the identification of additional effectors in L. pneumophila, we searched the genome sequence database for candidate genes.

As the modulation of organelle trafficking events appears to be important during intracellular multiplication, we looked for $L$. pneumophila effectors that resemble components of the SNARE system (19) and that might somehow disable or alter its function. Two such open reading frames (ORFs) were identified. Both exhibit limited sequence similarity to mammalian EEA1 and yeast USO1, proteins known to be involved in vesicle trafficking. Both Legionella ORFs are predicted to encode large regions of $\alpha$-helical coiled-coils, structures present in EEA1 and USO1 and also commonly found in SNAREs (table S1).

As there are no functional assays for these putative effectors, we investigated whether $L$. 
Bradley J., Dai Z.R., Erni R., Browning N., Graham G., Weber P., Smith J., Hutcheon I., Ishii H., Bajt S., Floss C., Stadermann F. and Sandford S. (2005) The astronomical 2175 angstrom feature in interplanetary dust particles. Science 307, 244-247. UCRL-JRNL-205703. 


\section{R E P O R T S}

torial humid belt in the Late Triassic was comparable to today's (Fig. 3), a conclusion that contrasts with some previous suggestions of a much more restricted or even dry equatorial belt in the Triassic $(33,34)$. Poleward motion can explain the generally drier northward and up-section facies pattern in the Mesozoic rift basins of eastern North America $(32,35)$ as this part of Pangea drifted out of the equatorial humid belt. At the same time, the up-section progression to more humid facies in the Fleming Fjord Formation $(36,37)$ and the overlying plant-bearing Kap Stewart Formation of latest Triassic and earliest Jurassic age (38) in the Jameson Land basin would reflect the drift of this area into the temperate humid belt.

We conclude that the congruence of the corrected paleomagnetic data from sedimentary rocks and independent data from igneous rocks ranging over thousands of kilometers and tens of millions of years indicates that a GAD field similar to that of the past $5 \mathrm{My}$ was operative at least $200 \mathrm{Ma}$ in the Late Triassic and earliest Jurassic. In particular, we see no evidence for a major octupole contribution in either the shapes of the distributions of directions in the sedimentary units or in the geographic distribution of site paleolatitudes. As indicated by other recent studies $(17,39-41)$, there is thus little empirical basis to invoke persistent departures from the GAD field, especially zonal octupole contributions, to address tectonic problems (4, 42, 43). Instead, our results suggest that inclination error in sedimentary rocks may be more prevalent than has been supposed, perhaps especially in cases where the magnetizations that have been isolated are most likely to represent a depositional remanence carried by hematite. The success of the E/I method (17) to determine the degree of flattening and to correct any bias in inclinations from the distribution of directions should provide motivation for more intensive sampling of sedimentary rock units and for making detailed data more accessible.

\section{References and Notes}

1. J. Hospers, Nature 173, 1183 (1954).

2. C. L. Johnson, C. G. Constable, Geophys. J. Int. 131, 643 (1997).

3. R. T. Merrill, P. L. McFadden, Phys. Earth Planet. Inter. 139, 171 (2003).

4. R. Van der Voo, T. H. Torsvik, Earth Planet. Sci. Lett. 187, 71 (2001)

5. J. Si, R. Van der Voo, Terra Nova 13, 471 (2001).

6. M. Westphal, Earth Planet. Sci. Lett. 117, 15 (1993).

7. R. F. King, Mon. Not. R. Astron. Soc. Geophys. Suppl. 7, 115 (1955).

8. L. Tauxe, D. V. Kent, Geophys. J. R. Astron. Soc. 77, $543(1984)$

9. P. Olsen, D. V. Kent, B. Cornet, W. K. Witte, R. W. Schlische, Geol. Soc. Am. Bull. 108, 40 (1996).

10. D. V. Kent, P. E. Olsen, W. K. Witte, J. Geophys. Res. 100, 14 (1995)

11. D. V. Kent, P. E. Olsen, J. Geophys. Res. 104, 12 (1999).

12. D. V. Kent, P. E. Olsen, Geol. Soc. Am. Bull. 109, 366 (1997).

13. D. V. Kent, P. E. Olsen, Earth Planet. Sci. Lett. 179, $311(2000)$
14. D. V. Kent, L. B. Clemmensen, Bull. Geol. Soc. Den. 42, 121 (1996).

15. W. R. Roest, S. P. Srivastava, Geology 17, 1000 (1989).

16. E. C. Bullard, J. E. Everett, A. G. Smith, Philos. Trans. R. Soc. London Ser. A 258, 41 (1965).

17. L. Tauxe, D. V. Kent, Geophys. Monogr. Am. Geophys. Union 145, 101 (2004).

18. C. G. Constable, R. L. Parker, J. Geophys. Res. 93, 11 (1988).

19. J. C. Briden, B. A. Daniels, J. Geol. Soc. London 156 317 (1999).

20. A. Marzoli et al., Science 284, 616 (1999).

21. M. Prevot, M. McWilliams, Geology 17, 1007 (1989).

22. J. P. Hodych, A. Hayatsu, Can. J. Earth Sci. 25, 1972 (1988).

23. J. Pohl, H. Soffel, Zeitschrift fur Geophysik 37, 857 (1971).

24. W. A. Robertson, Can. J. Earth Sci. 4, 641 (1967).

25. A. Larochelle, K. L. Currie, J. Geophys. Res. 72, 4163 (1967).

26. J. P. Hodych, G. R. Dunning, Geology 20, 51 (1992).

27. S. P. Kelley, J. G. Spray, Meteorit. Planet. Sci. 32, 629 (1997).

28. N. D. Opdyke, J. Geophys. Res. 66, 1941 (1961).

29. W. K. Witte, D. V. Kent, J. Geophys. Res. 96, 19 (1991).

30. J. L. Roy, Earth Planet. Sci. Lett. 14, 103 (1972).

31. E. I. Robbins, G. P. Wilkes, D. A. Textoris, in Triassic-Jurassic Rifting: North America and Africa, W. Manspeizer, Ed. (Elsevier, Amsterdam, 1988), pp. 649-682.

32. J. F. Hubert, K. A. Mertz, Geology 8, 516 (1980).

33. K. M. Wilson, D. Pollard, W. W. Hay, S. L. Thompson, C. N. Wold, Geol. Soc. Am. Spec. Pap. 288, 91 (1994).

34. J. T. Parrish, Journal of Geology 101, 215 (1993).
35. J. P. Smoot, Palaeogeogr. Palaeoclimatol. Palaeoecol. 84, 369 (1991).

36. L. B. Clemmensen, D. V. Kent, F. A. Jenkins Jr. Palaeogeogr. Palaeoclimatol. Palaeoecol. 140, 135 (1998).

37. L. B. Clemmensen, Geol. Surv. Greenland Bull. 136, 5 (1980).

38. J. C. McElwain, D. J. Beerling, F. I. Woodward, Science 285, 1386 (1999).

39. G. Muttoni et al., Earth Planet. Sci. Lett. 215, 379 (2003).

40. S. Gilder et al., Earth Planet. Sci. Lett. 206, 587 (2003).

41. W. Krijgsman, L. Tauxe, Earth Planet. Sci. Lett. 222, 685 (2004).

42. T. H. Torsvik, R. Van der Voo, Geophys. J. Int. 151, 771 (2002).

43. P. Rochette, D. Vandamme, Ann. Geofis. 44, 649 (2001)

44. NASA, Visible Earth, available at http://visibleearth. nasa.gov/.

45. T. J. Crowley, G. R. North, Oxford Monogr. Geol. Geophys. 18 (1991).

46. We thank $P$. Olsen for many discussions of the Triassic-Jurassic world and the reviewers for insightful comments. Supported by National Science Foundation grant nos. EAR-0310240 (D.V.K.) and EAR-0003395 (L.T.). This is LDEO contribution \#6700.

\section{Supporting Online Material}

www.sciencemag.org/cgi/content/full/307/5707/240/ DC1

Fig. S1

References and Notes

29 September 2004; accepted 9 December 2004 $10.1126 /$ science. 1105826

\title{
An Astronomical $2175 \AA$ Å Feature in Interplanetary Dust Particles
}

\author{
John Bradley, ${ }^{1 *}$ Zu Rong Dai, ${ }^{1}$ Rolf Erni, ${ }^{2}$ Nigel Browning, \\ Giles Graham, ${ }^{1}$ Peter Weber, ${ }^{1}$ Julie Smith, ${ }^{1}$ lan Hutcheon, ${ }^{1}$ \\ Hope Ishii, ${ }^{1}$ Sasa Bajt, ${ }^{1}$ Christine Floss, ${ }^{4}$ Frank Stadermann, ${ }^{4}$ \\ Scott Sandford ${ }^{5}$
}

\begin{abstract}
The 2175 angstrom extinction feature is the strongest (visible-ultraviolet) spectral signature of dust in the interstellar medium. Forty years after its discovery, the origin of the feature and the nature of the carrier(s) remain controversial. Using a transmission electron microscope, we detected a 5.7electron volt (2175 angstrom) feature in interstellar grains embedded within interplanetary dust particles (IDPs). The carriers are organic carbon and amorphous silicates that are abundant in IDPs and in the interstellar medium. These multiple carriers may explain the enigmatic invariant central wavelength and variable bandwidth of the astronomical 2175 angstrom feature.
\end{abstract}

Much of what is known about grains in space comes from spectral features observed in emission, polarization, and absorption (1-7). The $2175 \AA$ peak is by far the strongest feature observed in the ultraviolet (UV)-

\begin{abstract}
${ }^{1}$ Institute of Geophysics and Planetary Physics, Lawrence Livermore National Laboratory, Livermore, CA 94550, USA. ${ }^{2}$ Department of Chemical Engineering, University of California at Davis, CA 95616, USA. ${ }^{3}$ National Center for Electron Microscopy, Lawrence Berkeley National Laboratory, Berkeley, CA 94720, USA. ${ }^{4}$ Laboratory for Space Sciences, Washington University, St. Louis, MO 63130, USA. ${ }^{5}$ Astrophysics Branch, NASA-Ames Research Center, Moffett Field, CA 94035, USA.
\end{abstract}

*To whom correspondence should be addressed. E-mail: jbradley@igpp.ucllnl.org visible wavelength range along most lines of sight for which it can be measured (Fig. 1, A and B) (4-7). The feature is enigmatic: Its central wavelength is almost invariant, but its bandwidth varies from one line of sight to another, suggesting multiple carriers or a single carrier with variable properties. From interstellar abundances of the elements and typical UV transition strengths, the carrier is either oxygen-rich (e.g., oxides or silicates) or carbon-rich (e.g., graphite or organic compounds) $(1-4,8)$. We searched UV spectra of chondritic IDPs for an extinction feature near the $\sim 2175 \AA$ interstellar feature (Fig. 1). Materials similar to the two most abundant grain types seen in the 
interstellar medium (ISM), amorphous silicates and carbonaceous matter, are found in IDPs (Fig. 2) (9). The amorphous silicates are glass with embedded metal and sulfides (GEMS), some with nonsolar $\mathrm{O}$ isotopic compositions (10-13). The carbonaceous matter is a mixture of inorganic and organic carbon, and some of the organic materials exhibit nonsolar $\mathrm{D} / \mathrm{H},{ }^{15} \mathrm{~N} /{ }^{14} \mathrm{~N}$, and ${ }^{13} \mathrm{C} /{ }^{12} \mathrm{C}$ ratios comparable in magnitude to those observed in interstellar molecular clouds (13-15). The nonsolar isotopic signatures establish that these GEMS and carbonaceous subgrains are of interstellar origin.

We used a new-generation transmission electron microscope (TEM) equipped with a monochromator and high-resolution electron energy-loss spectrometer to measure UV spectral properties of portions of IDPs and standards $(16,17)$. The 0 - to $\sim 100-\mathrm{eV}$ region of an energy-loss spectrum, known as the valence electron energy-loss spectroscopy (VEELS) region (17), includes the $2175 \AA$ $(5.7 \mathrm{eV}) \mathrm{UV}$ spectral feature. We used VEELS because the submicrometer dimensions of the subgrains preclude measurement by conventional photoabsorption spectroscopy (PAS). The VEELS data were acquired under conditions in which the positions of VEELS and PAS features are comparable, but PAS typically has $\sim 10$ times better wavelength/energy resolution (18). A synchrotron light source was used to measure infrared (IR) spectral properties (17), and two NanoSIMS (secondary ion mass spectrometry) microprobes were used to measure the isotopic compositions of grains within the same IDPs (17).

A VEELS spectrum from the mineral talc $\left(\mathrm{Mg}_{3} \mathrm{Si}_{4} \mathrm{O}_{10}[\mathrm{OH}]_{2}\right)$ shows a peak position and bandwidth that match the photoabsorption feature of hydroxylated amorphous $\mathrm{Mg}_{2} \mathrm{SiO}_{4}$ (Fig. 1, C and D) (8), as well as the astronomical UV feature (Fig. 1, A, B, and D). VEELS spectra from carbonaceous grains in three IDPs exhibit a 5.7-eV feature with average bandwidth (full width at half maximum, FWHM) of $2.6 \mathrm{eV}\left(2.2 \mu \mathrm{m}^{-1}\right)$ (Fig. 3, $A$ to $C$ ). With increasing $\mathrm{O} / \mathrm{C}$ ratio, the strength of the $5.7-\mathrm{eV}$ feature increases and the peak of the volume plasmon (the broad peak between 10 and $28 \mathrm{eV}$ ) decreases in energy. Energy-loss $\mathrm{C}$ and $\mathrm{O}$ core scattering edges from the most O-rich regions exhibit a fine structure consistent with carbonyl (or hydroxyl) functional groups (19), and IR spectra there exhibit prominent $\mathrm{C}-\mathrm{H}$ stretch and $\mathrm{C}=\mathrm{O}$ features at $\sim 3.4 \mu \mathrm{m}$ and $\sim 5.9 \mu \mathrm{m}$, respectively (Fig. 4). Although the signal-tonoise ratio is marginal, because the IR spectrum was acquired from a $\sim 9-\mu \mathrm{m}^{2}$ area $\sim 0.1 \mu \mathrm{m}$ thick, the overall structure of the $\mathrm{C}-\mathrm{H}$ stretch feature between 2850 and $3100 \mathrm{~cm}^{-1}$ in L2036 G16 (and in other IDPs) is consistent with aliphatic groups bound to other molecules like polycyclic aromatic hydrocarbons (PAHs) (19-21). 1-Pyrene carboxaldehyde $\left(\mathrm{C}_{17} \mathrm{H}_{10} \mathrm{O}\right)$ exhibits the $\sim 5.7-\mathrm{eV}$ feature but pyrene $\left(\mathrm{C}_{16} \mathrm{H}_{10}\right)$, with no carbonyl group, produces a feature that is shifted to higher energy $(\sim 6.1$ eV) (Fig. 3, D and E). GEMS produce a 5.7$\mathrm{eV}$ feature with an average bandwidth (FWHM) of $2.9 \mathrm{eV}\left(2.5 \mu \mathrm{m}^{-1}\right)$, and the feature strength correlates with hydroxyl $\left(\mathrm{OH}^{-}\right)$content (Fig. 3, F to J). Thus, both organic compounds and amorphous silicates in IDPs may be carriers of a $5.7-\mathrm{eV}$ feature.

The central wavelength of the IDPs' $5.7-\mathrm{eV}$ VEELS feature matches the $2175 \AA$ astronomical feature, but the bandwidths are broader (Fig. 1). This extra breadth may result from the $\sim 10$ times lower energy resolution of VEELS (relative to PAS) and from the grains' physical state. The subgrains within the IDPs are no longer free-floating in the ISM, and the extent of their solid-state modification during their $\sim 4.5$-billion-year post-ISM lifetimes is unknown. At the very least they may have undergone significant aggregation into larger (50- to 500-nm-diameter) grains, and computer-modeling best fits to the

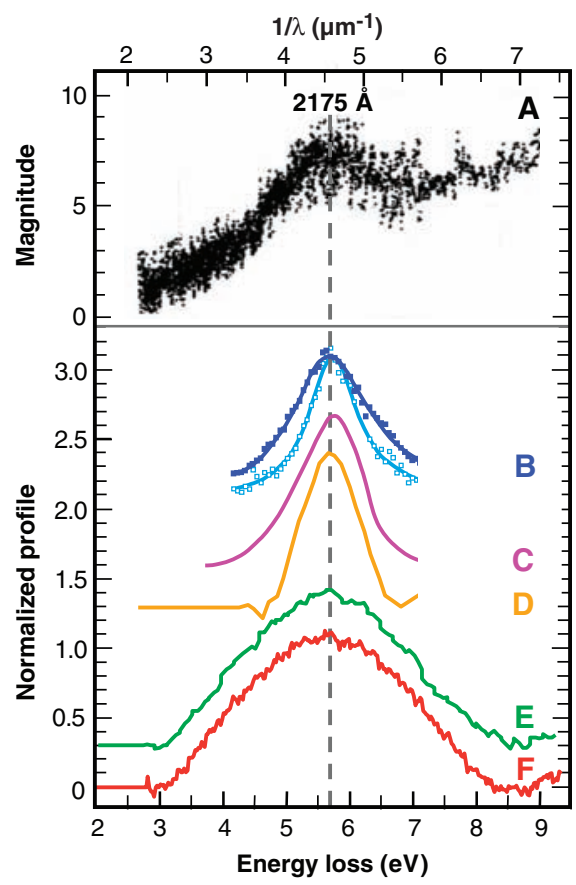

Fig. 1. Comparison of astronomical UV extinction features with laboratory UV and VEELS features. (A) The $2175 \AA$ interstellar extinction feature from two stars $\zeta$ and $\varepsilon$ Persei (5). (B) Broadest ( $\zeta$ Oph) and narrowest (HD 93028) profiles from 45 stars (6). (C) Photoabsorption spectrum from partially recrystallized hydroxylated amorphous magnesium silicate (8). (D) VEELS spectrum from (electron) irradiationdamaged talc $\left(\mathrm{Mg}_{3} \mathrm{Si}_{4} \mathrm{O}_{10}[\mathrm{OH}]_{2}\right)$. (E) VEELS spectrum from (organic) carbon in IDP L2047 D23. (F) VEELS spectrum from GEMS in W7013 E17. astronomical $2175 \AA$ feature are obtained with much smaller $(<15$-nm-diameter) grain sizes (1-4). Production of the interstellar $2175 \AA$ feature is generally thought to be due to electronic transitions associated with the surfaces of small grains, and modification of these surfaces by aggregation, for example, is expected to alter the spectral profile of the feature $(1,22)$. Finally, all IDPs have been pulse heated to $>350^{\circ} \mathrm{C}$ during atmospheric entry (23) where organic components and -OH-bearing grains (e.g., GEMS) are particularly susceptible to modification.

IR spectroscopy of selected areas rich in carbonaceous material in our IDPs indicates that carbonyl $(\mathrm{C}=\mathrm{O})$ groups are the likely carrier of much of the oxygen in the organic fractions (Fig. 4). The average bulk carbon content of IDPs is $\sim 12$ wt. \% (21), at least half of which is hydrocarbons. About $10 \%$ of the carbon is bonded to oxygen, either in carbonyl $(\mathrm{C}=\mathrm{O})$ groups or as aromatic chromophores bound to hydroxyl $(-\mathrm{OH})$ groups $(19,21,24)$. Multiple VEELS measurements sampling these regions show that the $5.7-\mathrm{eV}$ feature strength correlates with the $\mathrm{O} / \mathrm{C}$ ratio (Fig. 3, A to $\mathrm{E}$ ). This suggests that the $5.7-\mathrm{eV}$ feature produced by the carbonaceous subgrains in IDPs may be due to organic molecules (e.g., PAHs) with

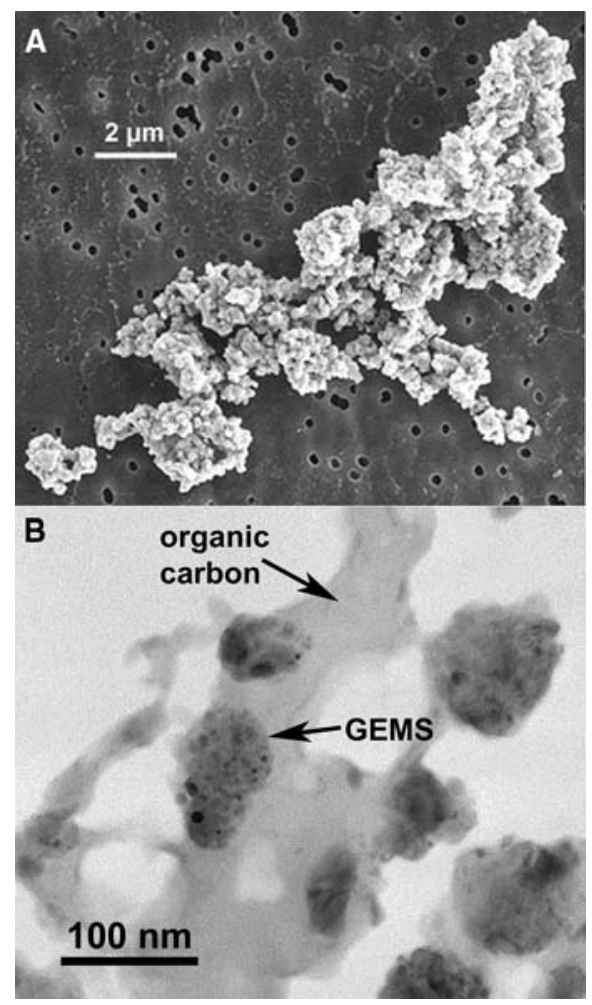

Fig. 2. (A) Secondary electron image of a typical chondritic IDP (RB12A). (B) A 200-keV brightfield transmission electron micrograph of organic carbon and GEMS within chondritic IDP L2009*E2. 


\section{R E P O R T S} grains in IDPs. (Left) Carbona500-nm-diameter grain, $\mathrm{O} / \mathrm{C}=$ 0.41. (B) L2047 D23, 800-nmdiameter grain with nonsolar ${ }^{14} \mathrm{~N} /{ }^{15} \mathrm{~N}$ ratio of $192 \pm 4(2 \sigma)$, $\mathrm{O} / \mathrm{C}=0.07$. (C) L2036-C18-F4, $\sim 400$-nm-diameter grain with correlated nonsolar ${ }^{12} \mathrm{C} /{ }^{13} \mathrm{C}=$ $80 \pm 2.4$ and ${ }^{14} \mathrm{~N} /{ }^{15} \mathrm{~N}=135 \pm$ 6.4 isotopic compositions, $\mathrm{O} / \mathrm{C}=$ 0.09. (D) Pyrene. (E) 1-Pyrene carboxaldehyde. (Right) GEMS: (F to H) W7013E17 (three GEMS, each 400 to $500 \mathrm{~nm}$ in diameter): (F) $\mathrm{O}_{\mathrm{ex}}=29.0$, (G) $\mathrm{O}_{\mathrm{ex}}=19.1$, (H) $\mathrm{O}_{\mathrm{ex}}=12.3$. (I) L2036-C24-13, $\sim 650$-nm-diameter grain, nonsolar ${ }^{16} \mathrm{O} /{ }^{17} \mathrm{O}=2262 \pm 108$, ${ }^{16} \mathrm{O} /{ }^{18} \mathrm{O}=403 \pm 8(9), \mathrm{O}_{\text {ex }}=2.3$. (J) L2036-C18-F4, $\sim 300-\mathrm{nm}-$ diameter grain, $\mathrm{O}_{\text {ex }}=1.6$. Dashed lines indicate $5.7 \mathrm{eV}$. The weak 10.5-eV feature in GEMS spectra (right) is a silicate exciton [the position of which overlaps Lyman- $\alpha$ emission (5)].
Fig. 3. VEELS spectra from subceous grains: (A) L2036-G16,

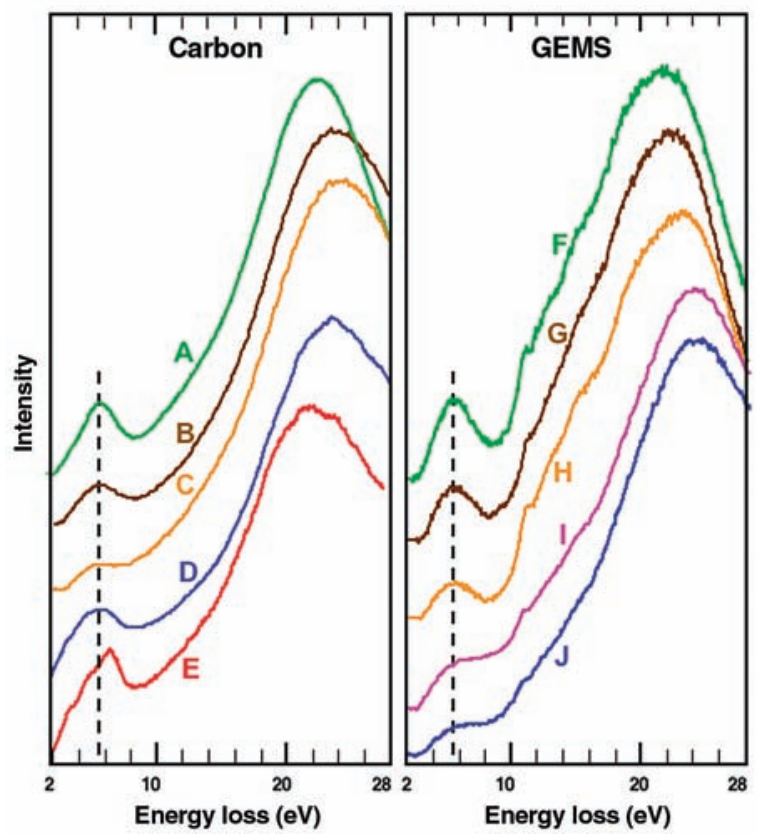

Fig. 4. IR spectrum from a $\sim 9-\mu \mathrm{m}^{2}$ region of IDP L2036-G16 (Fig. 3A). Peaks at 2850 to $2960 \mathrm{~cm}^{-1}$ are due to aliphatic $\mathrm{C}-\mathrm{H}$ stretch modes, and the peak at $\sim 1720 \mathrm{~cm}^{-1}$ is due to carbonyl $(C=O)$. Specimen thickness is $<100 \mathrm{~nm}$. (Insets) Electron energy-loss spectra recorded at $300 \mathrm{keV}$ (no monochromadouble $\pi^{*}$ edges (dashed lines) at $\sim 285.0 \mathrm{eV}$ and $286.5 \mathrm{eV}$ consistent with carbonyl $(C=O)$, and $(B)$ oxygen $\mathrm{K}$ edge showing a pre-edge at $\sim 531 \mathrm{eV}$ (arrow) associated with a $1 \mathrm{~s}$ to $\pi^{*}$ transition of oxygen and also consistent with carbonyl $(19,21,24)$. tor) of (A) carbon K-edge showing

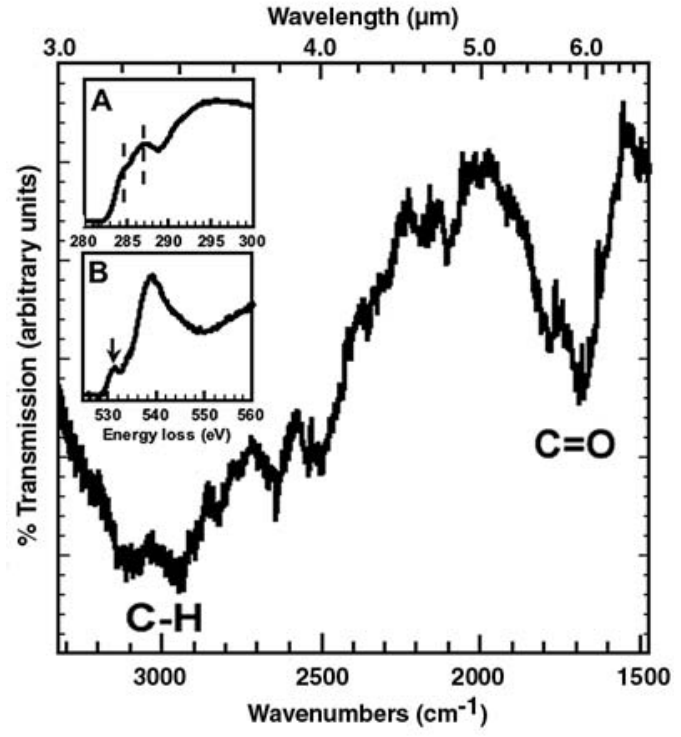

carbonyl functionality. VEELS measurements of unsubstituted and carbonylsubstituted pyrene are consistent with this hypothesis (Figs. 3, D and E). Other measurements suggest that substituted PAHs are abundant in IDPs $(24,25)$, and laboratory simulations of radiation processing in dense clouds have demonstrated that carbonyl groups can be added to PAHs under some interstellar conditions $(1,26)$. Carbonylsubstituted PAHs are also present in carbonaceous chondrites (27).

Organic compounds may also be responsible for the 5.7-eV feature from GEMS because, in addition to being coated with carbon, some GEMS contain carbonaceous matter within their interiors (28). However, the strength of the GEMS $5.7-\mathrm{eV}$ feature correlates with increasing $\mathrm{O}$ concentration (Fig. 3, F to J), implicating the inorganic Orich glassy matrices. Stoichiometric excesses of $\mathrm{O}\left(\mathrm{O}_{\mathrm{ex}}\right)$ observed in GEMS may be due primarily to hydroxyl ions $\left(\mathrm{OH}^{-}\right)$within their amorphous magnesium silicate matrices (11). Laboratory UV spectra of hydroxylated amorphous magnesium silicate particles exhibit an absorption feature at $2175 \AA$ that matches both the central wavelength and bandwidth of the interstellar feature $(8)$ and may be due to an electronic transition of hydroxyl ions in low-coordination sites $\left(\mathrm{OH}^{-}{ }_{\mathrm{LC}}\right)$ (Fig. 1C). In electron-irradiated talc $\left(\mathrm{Mg}_{3} \mathrm{Si}_{4} \mathrm{O}_{10}[\mathrm{OH}]_{2}\right)$ (Fig. 1D), the feature is also likely due to $\mathrm{OH}^{-}{ }_{\mathrm{LC}}$ because talc degrades (amorphizes) rapidly under the electron beam. Similarly, most of the hy- droxyl in GEMS is probably $\mathrm{OH}^{-}{ }_{\mathrm{LC}}$ because the glassy matrices are defect-rich from constant irradiation in space (11).

Carbonaceous and amorphous silicate grains exhibiting a $5.7-\mathrm{eV}(2175 \AA)$ UV feature in VEELS spectra have been identified in chondritic IDPs collected in the stratosphere. The species implicated as possible carriers for these features are carbonylcontaining molecules and hydroxylated amorphous silicates (GEMS). However, because carbonaceous material permeates some GEMS, molecular matter may be solely responsible for the $5.7-\mathrm{eV}$ feature. Both materials may have been produced by irradiation processing of dust in the ISM. Before this study, amorphous silicates, but not carbonyl compounds, were suggested as potential carriers of the astronomical $2175 \AA$ extinction feature. On the basis of our observations of IDPs, we cannot conclude that organic carbon and (hydroxylated) amorphous silicates are the only carriers of the astronomical feature. However, the identification of interstellar subgrains in IDPs (as evidenced by their isotopic compositions) that produce analogous features suggests that the carrier(s) of the interstellar feature may be present in IDPs. This finding provides new information for computational modeling, laboratory synthesis of analog grains, and laboratory (UV) photo-absorption measurements. It is also worth looking for a correlation of the interstellar $2175 \AA$ feature with IR carbonyl and hydroxyl bands, although lines-of-sight suitable for detecting a strong $2175 \AA$ feature are generally diffuse, whereas larger column densities are typically required for detection of weaker infrared bands. The presence of two potential carriers may bear on the variable bandwidth of the astronomical feature, with relative abundance or physical state of each component varying from one sight line to another. Amorphous silicates are ubiquitous throughout interstellar space, but oxidized (carbonylcontaining) PAHs have yet to be identified in the ISM, although they are indicated as a major product of irradiation of PAHs and are found in primitive meteorites. A variety of exotic carriers for the $2175 \AA$ peak have been proposed, including nanodiamonds, carbon onions, and fullerenes (1-4). However, organic carbon and amorphous silicates are more abundant in interstellar space, and cosmically abundant carriers are needed to explain the ubiquity of the $2175 \AA$ feature.

\section{References and Notes}

1. S. A. Sandford, Meteorit. Planet. Sci. 31, 449 (1996).

2. Th. Henning, F. Salama, Science 282, 2204 (1998).

3. B. T. Draine, Annu. Rev. Astron. Astrophys. 41, 241 (2003).

4. Th. Henning, C. Jäger, H. Mutschke, in Astrophysics of Dust, A. N. Witt, G. C. Clayton, B. T. Draine, Eds. [Astronomical Society of the Pacific (ASP) Conference Series, ASP, San Francisco, 2004], vol. 309, p. 603. 
5. T. P. Stecher, Astrophys. J. 157, L125 (1969)

6. E. L. Fitzpatrick, D. Massa, Astrophys. J. 307, 286 (1986)

7. E. L. Fitzpatrick, D. Massa, Astrophys. J. Suppl. 72 163 (1990)

8. T. M. Steel, W. W. Duley, Astrophys. J. 315, 337 (1987).

9. Collected IDPs are typically $<20 \mu \mathrm{m}$ in diameter and are composed mostly of aggregated submicrometer grains including silicates, sulfides, metal, and inorganic and organic compounds (e.g., PAHs) (10). They are from comets and asteroids, they include the most chemically and isotopically primitive meteoritic materials available for laboratory investigations, and they are logical materials in which to look for carriers of the $2175 \AA$ feature because they contain 10 to 100 times more interstellar material than the most primitive meteorites $(11,14)$.

10. J. P. Bradley, in Treatise of Geochemistry, A. M. Davis, H. D. Holland, K. K. Turekian, Eds. (Elsevier, Amsterdam, 2004), vol. 1, pp. 689-711.

11. J. P. Bradley, Science 265, 925 (1994).

12. S. Messenger, L. P. Keller, F. Stadermann, R. M. Walker, E. Zinner, Science 300, 105 (2003).

13. C. Floss, F. Stadermann, Lunar Planet. Sci. XXXV abstr. 1281 (2004).

14. C. Floss et al., Science 303, 1355 (2004).

15. S. Messenger, Nature 404, 968 (2000)

16. J. P. Bradley, Z. Dai, R. Erni, N. Browning, Lunar Planet. Sci. XXXV, abstr. 1433 (2004).
17. Materials and methods are available as supporting material on Science Online.

18. C. E. Brion, S. Daviel, R. Sodhi, A. P. Hitchcock in X-ray and Atomic Inner-Shell Physics-1982 B. Crasemann, Ed. (American Institute of Physics Conference Proceedings No. 94, American Institute of Physics, New York, 1982), pp. 429-446.

19. G. J. Flynn, L. P. Keller, C. Jacobsen, S. Wirick, Adv. Space Res. 33, 57 (2003).

20. S. A. Sandford et al., Astrophys. J. 371, 607 (1991).

21. L. P. Keller, S. Messenger, G. J. Flynn, S. Clemett, S. Wirick, C. Jacobsen, Geochim. Cosmochim. Acta 68 2577 (2004).

22. B. T. Draine, in Interstellar Dust, L. J. Allamandola, A. G. G. M. Tielens, Eds. (Kluwer, Dordrecht, Netherlands, 1989), pp. 313-327.

23. S. A. Sandford, J. P. Bradley, Icarus 82, 146 (1989).

24. L. P. Keller, K. L. Thomas, D. S. McKay, in Analysis of Interplanetary Dust, M. E. Zolensky, T. L. Wilson, F. J. M. Rietmeijer, G. J. Flynn, Eds. (American Institute of Physics Conference Proceedings, American Institute of Physics, New York, 1994), vol. 310, pp. 51-87.

25. S. J. Clemett, C. R. Maechling, R. N. Zare, P. D. Swan, R. M. Walker, Science 262, 721 (1993).

26. M. P. Bernstein et al., Astrophys. J. 582, L25 (2003).

27. J. R. Cronin, S. Chang, in The Chemistry of Life's Origins, J. M. Greenberg, C. X. Mendoza-Gomez, V. Pirronello, Eds. (Kluwer, Dordrecht, Netherlands, 1993), pp. 209-258.
28. D. E. Brownlee, D. J. Joswiak, J. P. Bradley, J. C. Gezo H. G. M. Hill, Lunar Planet. Sci. XXXI, 1921 (2000).

29. This research was in part performed under the auspices of the U.S. Department of Energy (DOE), National Nuclear Security Administration, by the University of California under contract W-7405-Eng-48. The electron microscopy performed at the National Center for Electron Microscopy and infrared microspectroscopy performed at the Advanced Light Source (ALS) at Lawrence Berkeley National Laboratory are supported by the Director, Office of Science, Office of Basic Energy Sciences, Materials Sciences Division, DOE, under contract DE-AC03-76SF00098. This work was supported by NASA grants NAG5-10632 and NAG5-10696 (to J.B.) and grant NNG04GG49G (C.F.). We thank L. Nittler for image-processing software development and $\mathrm{M}$. C. Martin and ALS personnel for beamline support. We also thank M. Bernas for performing the focused ion beam instrument work. We gratefully acknowledge discussions with M. Bernstein, D. Brownlee, B. Draine, Th. Henning, X. Tielens, and F. Salama.

Supporting Online Material

www.sciencemag.org/cgi/content/full/307/5707/244/ DC1

Materials and Methods

25 October 2004; accepted 10 December 2004 10.1126/science. 1106717

\title{
Retinoic Acid Signaling Restricts the Cardiac Progenitor Pool
}

\author{
Brian R. Keegan, ${ }^{1}$ Jessica L. Feldman, ${ }^{1}$ Gerrit Begemann, ${ }^{2}$ \\ Philip W. Ingham, ${ }^{3}$ Deborah Yelon ${ }^{1 *}$
}

\begin{abstract}
Organogenesis begins with specification of a progenitor cell population, the size of which provides a foundation for the organ's final dimensions. Here, we present a new mechanism for regulating the number of progenitor cells by limiting their density within a competent region. We demonstrate that retinoic acid signaling restricts cardiac specification in the zebrafish embryo. Reduction of retinoic acid signaling causes formation of an excess of cardiomyocytes, via fate transformations that increase cardiac progenitor density within a multipotential zone. Thus, retinoic acid signaling creates a balance between cardiac and noncardiac identities, thereby refining the dimensions of the cardiac progenitor pool.
\end{abstract}

Generation of the proper number of organ progenitor cells is likely to involve interplay between inductive and repressive signaling pathways. Key inductive mechanisms have been identified for many organs, including the heart, but mechanisms for repressing progenitor fate assignment are poorly understood. Several factors, including Bmp2, Fgf8, Nodal, and Wnt11, are implicated in promoting the initial selection of myocardial progenitor cells from a multipotential popu-

\footnotetext{
${ }^{1}$ Developmental Genetics Program, Skirball Institute of Biomolecular Medicine, and Department of Cell Biology, New York University School of Medicine, New York, NY 10016, USA. '2Lehrstuhl Zoology/ Evolutionary Biology, University of Konstanz, 78457 Konstanz, Germany. ${ }^{3}$ Centre for Developmental Genetics, Department of Biomedical Science, University of Sheffield School of Medicine, Firth Court, Western Bank, Sheffield S10 2TN, UK

*To whom correspondence should be addressed. E-mail: yelon@saturn.med.nyu.edu
}

lation (1). Although convergence of inductive signals might be sufficient to delimit the number of progenitor cells, opposing signals could also be necessary to restrict myocardial specification. Prior studies have suggested mechanisms for inhibiting cardiomyocyte differentiation within the anterior lateral plate mesoderm (ALPM), by means of Notch signaling (2) or interactions with the notochord (3), but little is known about whether repressive pathways limit the initial assignment of myocardial identity

We find that reduction of retinoic acid (RA) signaling causes formation of an excess of cardiomyocytes. The zebrafish mutation neckless (nls) disrupts function of the retinaldehyde dehydrogenase 2 gene (raldh2), which controls a rate-limiting step in RA synthesis $(4,5)$. nls mutants exhibit an increased number of cells expressing $n k x 2.5$, a marker of the bilateral populations of precardiac mesoderm within the ALPM (Fig. 1A). Although $n k x 2.5$ expression appears expanded in anterior posterior, and lateral directions (Fig. 1, A and B), we do not observe an increase in the overall size of the ALPM in $n l s$ mutants (fig. S1A). As myocardial differentiation proceeds, $n l s$ mutants exhibit a surplus of cardiomyocytes, identifiable by their expression of cardiac myosin light chain 2 ( cmlc2) (Fig. 1B; fig. S1B). Formation of this myocardial surplus depends on the conventional myocardial differentiation pathway (1), which requires the activity of the growth factor Fgf8 and the transcription factors Hand2 and Gata5 (fig. S2). Consistent with a repressive influence of RA on cardiomyocyte formation, exposure to the pan-retinoic acid receptor (RAR) antagonist BMS189453 $(6,7)$ causes expansion of $n k x 2.5$ and $c m l c 2$ expression (Fig. 1, A and B). Conversely, exposure to exogenous RA results in a reduced number of cardiomyocytes (fig. S1C) (8). Together, these data demonstrate that cardiomyocyte population size within the ALPM is inversely related to the level of RA signaling.

The raldh2 gene is expressed throughout early zebrafish embryogenesis $(4,5)$ : In the blastula, raldh2 is found at the embryonic margin; during gastrulation, raldh2 is in involuting mesendoderm; and, after gastrulation, raldh2 is in both lateral and paraxial mesoderm. To investigate when RA influences cardiomyocyte number, we tested the effectiveness of BMS189453 during different time intervals, initiating exposure at various stages and later assessing $n k \times 2.5$ or $c m l c 2$ expression (Fig. 1, C and D; fig. S3). Addition of BMS189453 before gastrulation [40\% epiboly, 5 hours post fertilization (hpf)] causes a myocardial expansion, whereas addition of BMS189453 during gastrulation (75\% epiboly, $8 \mathrm{hpf}$ ) results in a more modest increase. 